\title{
GALOIS ACTION ON KNOTS I: ACTION OF THE ABSOLUTE GALOIS GROUP
}

\author{
HIDEKAZU FURUSHO
}

\begin{abstract}
Our aim of this and subsequent papers is to enlighten (a part of, presumably) arithmetic structures of knots. This paper introduces a notion of profinite knots which extends topological knots and shows its various basic properties. Particularly an action of the absolute Galois group of the rational number field on profinite knots is rigorously established, which is attained by our extending the action of Drinfeld's Grothendieck-Teichmüller group on profinite braid groups into on profinite knots.
\end{abstract}

\section{Contents}

0. Introduction

1. Profinite braids

2. Profinite knots

2.2. Pro-l knots 23

2.3. Action of the absolute Galois group 28

Appendix A. Two-bridge profinite knots 39

Appendix B. Profinite framed knots $\quad 40$

References

\section{INTRODUCTION}

It is known that there are analogies between algebraic number theory and 3dimensional topology. It is said that Mazur and Manin among others spotted them in 1960's and, after a long silence, in 1990's, Kapranov and Reznikov ( Kap, R and their lecture in MPI) who took up their ideas and explored them jointly and Morishita [Mo] whose works started independently in a more sophisticated aspect, settled the new area of mathematics, arithmetic topology. Lots of analogies between algebraic number theory and 3-dimensional topology are suggested in arithmetic topology, however, as far as we know, no direct relationship seems to be known. Our attempt of this and subsequent [F2] papers is to give a direct one particularly between Galois groups and knots.

A profinite tangle diagram, a profinite analogue of an oriented tangle diagram, is introduced in Definition 2.3 as a consistent finite sequence of fundamental profinite tangle diagrams; symbols of three types $A, \widehat{B}$ and $C$ (Definition 2.2). A profinite

Date: June 27, 2015.

2000 Mathematics Subject Classification. Primary 14G32 ; Secondary 11R, 57M25. 
knot diagram, a profinite analogue of an oriented knot diagram, is defined by a profinite tangle diagram without endpoints and with a single connected component in Definition 2.4. A profinite version of Turaev moves is given in our Definition 2.8 (T1)-(T6), which determines an equivalence, called isotopy, for profinite tangle diagrams. The set $\widehat{\mathcal{T}}$ of profinite tangles means the quotient of the set of profinite tangle diagrams by the equivalence and the set $\widehat{\mathcal{K}}$ of profinite knots is the subspace of $\widehat{\mathcal{T}}$ consisting of isotopy classes of profinite knot diagrams (Definition [2.9). Our first theorem is

Theorem A (Theorem 2.11 and 2.16).

(1). The space $\widehat{\mathcal{K}}$ carries a structure of a topological commutative monoid whose product is given by the connected sum (2.1).

(2). Let $\mathcal{K}$ denote the set of isotopy classes of (topological) oriented knots. Then there is a natural map

$$
h: \mathcal{K} \rightarrow \widehat{\mathcal{K}} .
$$

The map is with dense image and is a monoid homomorphism with respect to the connected sum.

The map $h$ is naturally conjectured to be injective (Conjecture 2.12). It is because, if it is not injective, the Kontsevich invariant fail to be a perfect knot invariant (cf. Remark 2.13 and 2.34).

The topological group Frac $\widehat{\mathcal{K}}$ of profinite knots is introduced as the group of fraction of the topological monoid $\widehat{\mathcal{K}}$ in Definition 2.35. A continuous action of the profinite Grothendieck-Teichmüller group $\widehat{G T}$ [Dr2] on Frac $\widehat{\mathcal{K}}$ is rigorously established in Definition 2.39 - Theorem 2.41. By using the inclusion from the absolute Galois group $G_{\mathbb{Q}}$ of the rational number field $\mathbb{Q}$ into $\widehat{G T}$, our second theorem is obtained.

Theorem B (Theorem 2.45).

Fix an embedding from the algebraic closure $\overline{\mathbb{Q}}$ of the rational number field $\mathbb{Q}$ into the complex number field $\mathbb{C}$. Then the group Frac $\widehat{\mathcal{K}}$ of profinite knots admits a nontrivial topological $G_{\mathbb{Q}}$-module structure. Namely, there is a non-trivial continuous Galois representation on profinite knots

$$
\rho_{0}: G_{\mathbb{Q}} \rightarrow \text { Aut Frac } \widehat{\mathcal{K}} .
$$

It is explained that particularly the complex conjugation sends each knot to its mirror image (Example 2.46). The validity of a knot analogue of the so-called Belyı̌'s theorem, i.e. the injectivity of $\rho_{0}$, is posed in Problem 2.49 Several projects and problems on the Galois action are posted in the end of this paper.

Our construction of the Galois action could be said as a lift of the action of Kassel-Turaev [KT given in a proalgebraic setting into a profinite setting. Our Galois action on knots might also be related to the 'Galois relations' suggested in Gannon-Walton GW]. Our discussion on profinite knots in this paper may be linked to Mazur's discussion on profinite equivalence of knots in $\mathrm{Ma}$

The contents of the paper is as follows. \$1 is devoted to a review of Drinfeld's

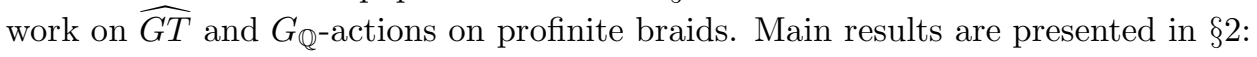
The ABC-construction of profinite knots and their basic properties are introduced in 2.1. We also introduce and discuss the notion of pro-l knots in 2.2 , It serves for our arguments in $\$ 2.3$ where an action of the absolute Galois group on profinite knots is established. In Appendix $\mathrm{A}$ a two-bridge profinite knot, a profinite knot 
with a specific type, is introduced and its Galois behavior is investigated. A profinite analogue of a framed knot is introduced and the above two theorems are extended into those for profinite framed knots in Appendix B.

\section{Profinite BRAids}

This section is a review mainly on Drinfeld's work Dr2 of his profinite GrothendieckTeichmüller group $\widehat{G T}$ and its action on profinite braids. Definitions of the profinite braid group $\widehat{B}_{n}$ and the absolute Galois group $G_{\mathbb{Q}}$ are recalled in Example 1.4. The definition of $\widehat{G T}$ is presented in Definition [1.6. In Theorem 1.9, it is explained that $\widehat{G T}$ contains $G_{\mathbb{Q}}$. The action of $\widehat{G T}$ on $\widehat{B}_{n}$ is explained in Theorem 1.12 Specific properties of the action which will be required to next section are shown in Proposition 1.16 and Proposition 1.18.

Definition 1.1. The Artin braid group $B_{n}$ with $n$-strings $(n \geqslant 2)$ is the group generated by $\sigma_{i}(1 \leqslant i \leqslant n-1)$ with two relations

$$
\begin{aligned}
& \sigma_{i} \sigma_{i+1} \sigma_{i}=\sigma_{i+1} \sigma_{i} \sigma_{i+1} \\
& \sigma_{i} \sigma_{j}=\sigma_{j} \sigma_{i} \quad \text { if } \quad|i-j|>1 .
\end{aligned}
$$

The unit of $B_{n}$ is denoted by $e_{n}$. For $n=1$, put $B_{1}=\left\{e_{1}\right\}$ : the trivial group. The pure braid group $P_{n}$ with $n$-strings is the kernel of the natural projection from $B_{n}$ to the symmetric group $\mathfrak{S}_{n}$ of degree $n$.

When $n=2$, there is an identification $B_{2} \simeq \mathbb{Z}$ and the subgroup $P_{2}$ corresponds to $2 \mathbb{Z}$ under the identification. When $n=3, P_{3}$ contains a free group $F_{2}$ generated by $\sigma_{1}^{2}$ and $\sigma_{2}^{2}$.

Notation 1.2. The generator $\sigma_{i}$ in $B_{n}$ is depicted as in Figure 1.1. And for $b$ and $b^{\prime} \in B_{n}$, we draw the product $b \cdot b^{\prime} \in B_{n}$ as in Figure 1.2 (the order of product $b \cdot b^{\prime}$ is chosen to combine the bottom endpoints of $b$ with the top endpoints of $\left.b^{\prime}\right)$.

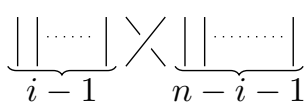

FiguRE 1.1. $\sigma_{i}$

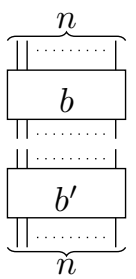

FIGURE $1.2 . b \cdot b^{\prime}$

For $1 \leqslant i<j \leqslant n$, special elements

$$
x_{i, j}=x_{j, i}=\left(\sigma_{j-1} \cdots \sigma_{i+1}\right) \sigma_{i}^{2}\left(\sigma_{j-1} \cdots \sigma_{i+1}\right)^{-1}
$$

generate $P_{n}$. For $1 \leqslant a \leqslant a+\alpha<b \leqslant b+\beta \leqslant n$, we define

$$
\begin{aligned}
x_{a \cdots a+\alpha, b \cdots b+\beta}:= & \left(x_{a, b} x_{a, b+1} \cdots x_{a, b+\beta}\right) \cdot\left(x_{a+1, b} x_{a+1, b+1} \cdots x_{a+1, b+\beta}\right) \\
& \cdots\left(x_{a+\alpha, b} x_{a+\alpha, b+1} \cdots x_{a+\alpha, b+\beta}\right) \in P_{n} .
\end{aligned}
$$

They are drawn in Figure 1.3 and 1.4

Next we briefly review the definition and a few examples of profinite groups. 


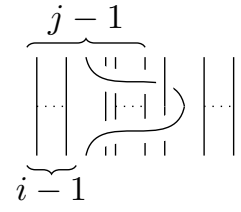

FigURE 1.3. $x_{i j}$

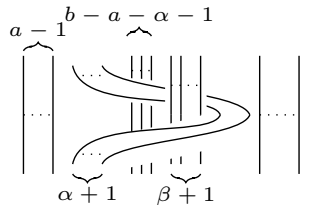

FigURE 1.4. $x_{a \cdots a+\alpha, b \cdots b+\beta}$

Definition 1.3. A topological group $G$ is called a profinite group if it is a projective $\operatorname{limit} \lim _{\complement} G_{i}$ of a projective system of finite groups $\left\{G_{i}\right\}_{i \in I}$. For a discrete group $\Gamma$, its profinite completion $\widehat{\Gamma}$ is the profinite group defined by the projective limit

$$
\widehat{\Gamma}=\lim _{\longleftarrow} \Gamma / N
$$

where $N$ runs over all normal subgroups of $\Gamma$ with finite indices.

For profinite groups, consult [RZ for example. We note there is a natural homomorphism $\Gamma \rightarrow \widehat{\Gamma}$. In the paper, we employ the same symbol when we express the image of elements of $\Gamma$ by the map if there is no confusion.

Example 1.4. (1). The set $\widehat{\mathbb{Z}}$ of profinite integers is the profinite completion of $\mathbb{Z}$. There is an identification $\widehat{\mathbb{Z}} \simeq \prod_{p} \mathbb{Z}_{p}$. Here $p$ runs over all primes and $\mathbb{Z}_{p}$ stands for the ring of $p$-adic integers.

(2). The absolute Galois group $G_{\mathbb{Q}}$ of the rational number field $\mathbb{Q}$ is the profinite group

$$
G_{\mathbb{Q}}=\operatorname{Gal}(\overline{\mathbb{Q}} / \mathbb{Q}):=\lim _{\longleftarrow} \operatorname{Gal}(F / \mathbb{Q}) .
$$

Here the limit runs over all finite Galois extension $F$ of $\mathbb{Q}$ and $\operatorname{Gal}(F / \mathbb{Q})$ means its Galois group.

(3). The profinite braid group $\widehat{B}_{n}$ means the profinite completion of $B_{n}$. It contains the profinite pure braid group $\widehat{P}_{n}$ (the profinite completion of $P_{n}$ ), which is equal to the kernel of the natural projection $\widehat{B}_{n} \rightarrow \mathfrak{S}_{n}$. It is known that both $B_{n}$ and $P_{n}$ are residually finite, i.e, their natural maps are both injective;

$$
B_{n} \hookrightarrow \widehat{B}_{n} \quad \text { and } \quad P_{n} \hookrightarrow \widehat{P}_{n} .
$$

When $n=2$, we have $\widehat{B}_{2} \simeq \widehat{\mathbb{Z}}$. For profinite braid groups, we employ the same figures as in Notation [1.2 to express such elements. Further we also depict $\sigma_{i}^{c} \in \widehat{B}_{n}$ $(c \in \widehat{\mathbb{Z}})$ as Figure 1.5

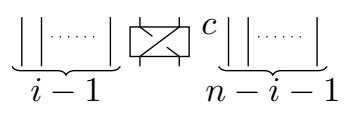

Figure 1.5. $\sigma_{i}^{c} \in \widehat{B}_{n}(c \in \widehat{\mathbb{Z}})$

To state the definition of $\widehat{G T}$, we need fix several notations.

Notation 1.5. Let $F_{2}$ be the free group of rank 2 with two variables $x$ and $y$ and $\widehat{F}_{2}$ be its profinite completion. For any $f \in \widehat{F}_{2}$ and any group homomorphism $\tau: \widehat{F}_{2} \rightarrow G$ sending $x \mapsto \alpha$ and $y \mapsto \beta$, the symbol $f(\alpha, \beta)$ stands for the image $\tau(f)$. 
Particularly for the (actually injective) group homomorphism $\widehat{F}_{2} \rightarrow \widehat{P}_{n}$ sending $x \mapsto$ $x_{a \cdots a+\alpha, b \cdots b+\beta}$ and $y \mapsto x_{b \cdots b+\beta, c \cdots c+\gamma}(1 \leqslant a \leqslant a+\alpha<b \leqslant b+\beta<c \leqslant c+\gamma \leqslant n)$, the image of $f \in \widehat{F}_{2}$ is denoted by $f_{a \cdots a+\alpha, b \cdots b+\beta, c \cdots c+\gamma}$.

The profinite Grothendieck-Teichmüller group $\widehat{G T}$ which is a main character of our paper is defined by Drinfeld Dr2 to be a profinite subgroup of the topological automorphism group of $\widehat{F}_{2}$.

Definition 1.6 (Dr2]). The profinite Grothendieck-Teichmüller group $1 \widehat{\mid} \widehat{G T}$ is the profinite subgroup of Aut $\widehat{F}_{2}$ defined by

$$
\widehat{G T}:=\left\{\begin{array}{l|l}
\sigma \in \operatorname{Aut} \widehat{F}_{2} \mid \begin{array}{l}
\sigma(x)=x^{\lambda}, \sigma(y)=f^{-1} y^{\lambda} f \text { for some }(\lambda, f) \in \widehat{\mathbb{Z}}^{\times} \times \widehat{F}_{2} \\
\text { satisfying the three relations below. }
\end{array}
\end{array}\right\}
$$

$$
\begin{gathered}
f(x, y) f(y, x)=1 \quad \text { in } \widehat{F}_{2} \\
f(z, x) z^{m} f(y, z) y^{m} f(x, y) x^{m}=1 \quad \text { in } \widehat{F}_{2} \text { with } z=(x y)^{-1} \text { and } m=\frac{\lambda-1}{2} \\
f_{1,2,34} f_{12,3,4}=f_{2,3,4} f_{1,23,4} f_{1,2,3} \quad \text { in } \widehat{P}_{4} .
\end{gathered}
$$

Remark 1.7. (1). In some literatures, (1.2), (1.3) and (1.4) are called 2-cycle, 3-cycle and 5-cycle relation respectively. The author often calls (1.2) and (1.3) by two hexagon equations and (1.4) by one pentagon equation because they reflect the three axioms, two hexagon and one pentagon axioms, of braided monoidal (tensor) categories [JS. We remind that (1.4) represents

$$
f\left(x_{12}, x_{23} x_{24}\right) f\left(x_{13} x_{23}, x_{34}\right)=f\left(x_{23}, x_{34}\right) f\left(x_{12} x_{13}, x_{24} x_{34}\right) f\left(x_{12}, x_{23}\right) \text { in } \widehat{P}_{4} .
$$

In several literatures such as [1] and [S], the equation (1.4) is replaced by a different (more symmetric) formulation.

(2). For the proalgebraic Grothendieck-Teichmüller group $G T(\mathbf{k})$ in Dr2] (k: an algebraically closed field of characteristic 0 ), it is shown that its one pentagon equation implies its two hexagon equations in [F1]. But as for our profinite group $\widehat{G T}$, it is not known if the pentagon equation (1.4) implies two hexagon equations (1.2) and (1.3).

(3). We remark that each $\sigma \in \widehat{G T}$ determines a pair $(\lambda, f)$ uniquely because the pentagon equation (1.4) implies that $f$ belongs to the topological commutator subgroup of $\widehat{F}_{2}$. By abuse of notation, we occasionally express the pair $(\lambda, f)$ to represent $\sigma$ and denote as $\sigma=(\lambda, f) \in \widehat{G T}$. The above set-theoretically defined $\widehat{G T}$ forms indeed a profinite group whose product is induced from that of Aut $\widehat{F}_{2}$ and is given by 2

$$
\left(\lambda_{2}, f_{2}\right) \circ\left(\lambda_{1}, f_{1}\right)=\left(\lambda_{2} \lambda_{1}, f_{2} \cdot f_{1}\left(x^{\lambda_{2}}, f_{2}^{-1} y^{\lambda_{2}} f_{2}\right)\right) .
$$

The next lemma will be used later.

\footnotetext{
${ }^{1}$ It is named after Grothendieck's project of un jeu de Teichmüller-Lego posted in Esquisse d'un programme G2. A construction of such group was suggested there though Drinfeld came to the group independently in his subsequent works Dr1 Dr2] on deformation of specific type of quantum groups.

2 For our purpose, we change the order of the product in Dr2].
} 
Lemma 1.8. Let $p_{i}: \widehat{P}_{3} \rightarrow \widehat{P}_{2}(=\widehat{\mathbb{Z}})(i=1,2,3)$ denote the map of omission of $i$-th strand in $\widehat{P}_{3}$. Let $(\lambda, f) \in \widehat{G T}$. Then $p_{i}\left(f_{1,2,3}\right)=0(i=1,2,3)$.

Proof. It is easy because $f$ belongs to the commutator of $\widehat{F}_{2}$ as mentioned above.

One of the important property of the profinite Grothendieck-Teichmüller group $\widehat{G T}$ is that it contains the absolute Galois group $G_{\mathbb{Q}}$.

Theorem 1.9 ([Dr2, [2] $)$. Fix an embedding from $\overline{\mathbb{Q}}$ into $\mathbb{C}$. Then there is an embedding

$$
G_{\mathbb{Q}} \hookrightarrow \widehat{G T} .
$$

We briefly review its proof below.

Proof. As is explained in [12], an action of the absolute Galois group on $\widehat{F}_{2}$, i.e.

$$
G_{\mathbb{Q}} \rightarrow \text { Aut } \widehat{F}_{2}
$$

is derived from the action on the profinite (scheme-theoretical, cf. G1]) fundamental group $\widehat{\pi}_{1}\left(\mathbf{P}_{\widehat{\mathbb{Q}}}^{1} \backslash\{0,1, \infty\}, \overrightarrow{01}\right)$ of the algebraic curve $\mathbf{P}_{\mathbb{Q}}^{1} \backslash\{0,1, \infty\}$ with Deligne's De tangential base point $\overrightarrow{01}$ (this is achieved in the method explained in Remark 1.14 below). The so-called Belyı's theorem $\mathrm{Be}$ claims that the map (1.7) is injective:

$$
G_{\mathbb{Q}} \hookrightarrow \text { Aut } \widehat{F}_{2} \text {. }
$$

The equations (1.2)-(1.4) are checked for $\sigma \in G_{\mathbb{Q}}$ in [Dr2, IM], which means that $G_{\mathbb{Q}}$ is contained in $\widehat{G T} \subset \operatorname{Aut} \widehat{F}_{2}$.

Example 1.10. Fix an embedding from $\overline{\mathbb{Q}}$ into $\mathbb{C}$. Then the complex conjugation sending $z \in \mathbb{C}$ to $\bar{z} \in \mathbb{C}$ determines an element $\varsigma_{0} \in G_{\mathbb{Q}}$. It is mapped to the pair $(-1,1) \in \widehat{G T}$ by (1.6).

Asking its surjectivity on the injection (1.6) is open for many years:

Problem 1.11. Is $G_{\mathbb{Q}}$ equal to $\widehat{G T}$ ?

The following Drinfeld's Dr2 $\widehat{G T}$-action on $\widehat{B}_{n}$ (a detailed description is also given in [IM]) plays a fundamental role of our results, $\widehat{G T}$-action on knots.

Theorem $1.12\left([\overline{\mathrm{Dr} 2},[\mathrm{IM}])\right.$. Let $n \geqslant 2$. There is a continuous $\widehat{G T}$-action on $\widehat{B}_{n}$

$$
\rho_{n}: \widehat{G T} \rightarrow \text { Aut } \widehat{B}_{n}
$$

given by

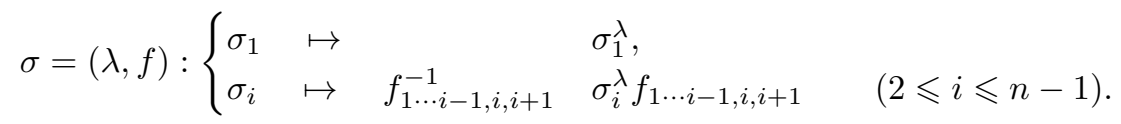

We denote $\rho_{n}(\sigma)(b)$ simply by $\sigma(b)$ when there is no confusion.

Remark 1.13. (1). According to the method to calculate the action in Dr2] (explicitly presented in the appendix of [IM]), particularly we have

$$
\begin{aligned}
\left(\sigma_{1} \cdots \sigma_{i}\right) & \mapsto f_{[1],[i],[n-i-1]}^{-1} \cdot\left(\sigma_{1} \cdots \sigma_{i}\right) \cdot x_{1 \cdots i, i+1}^{m}, \\
\left(\sigma_{i} \cdots \sigma_{1}\right) & \mapsto x_{1 \cdots i, i+1}^{m} \cdot\left(\sigma_{i} \cdots \sigma_{1}\right) \cdot f_{[1],[i],[n-i-1]} .
\end{aligned}
$$


Here $m=\frac{\lambda-1}{2}$ and for $f_{[1],[i],[n-i-1]}$, see (1.13).

(2). We note that $\rho_{n}$ is injective when $n \geqslant 4$.

By Theorem 1.9 and Theorem 1.12, we obtain the absolute Galois representation

$$
\rho_{n}: G_{\mathbb{Q}} \rightarrow \text { Aut } \widehat{B}_{n} .
$$

The below is an algebraic-geometrical interpretation of the Galois action in terms of Grothendieck's theory [G1] on profinite (scheme-theoretical) fundamental groups.

Remark 1.14. We have a well-known identification between the braid group $B_{n}$ with the topological fundamental group $\pi_{1}\left(X_{n}(\mathbb{C}), *\right)$. Here $X_{n}(\mathbb{C})=\operatorname{Conf}_{\mathfrak{S}_{n}}^{n}(\mathbb{C})$ means the quotient of the configuration space

$$
\operatorname{Conf}^{n}(\mathbb{C})=\left\{\left(z_{1}, \ldots, z_{n}\right) \in \mathbb{C}^{n} \mid z_{i} \neq z_{j}(i \neq j)\right\}
$$

by the symmetric group $\mathfrak{S}_{n}$ action and $*$ is a basepoint.

Let $\widehat{\pi}_{1}\left(X_{n} \times \overline{\mathbb{Q}}, *\right)$ denote the profinite (scheme-theoretical) fundamental group of $X_{n} \times \overline{\mathbb{Q}}$ in the sense of Grothendieck G1. Here the scheme $X_{n}$ means the $\mathbb{Q}$ structure of $X_{n}(\mathbb{C})$ and $*$ is a basepoint defined over $\overline{\mathbb{Q}}$ in the sense of loc.cit. Fix an embedding from $\overline{\mathbb{Q}}$ into $\mathbb{C}$, then, by the so-called Riemann's existence theorem (lo.cit. VII.Théorème 5.1), the group $\widehat{\pi}_{1}\left(X_{n} \times \overline{\mathbb{Q}}, *\right)$ is identified with the profinite completion of $\pi_{1}\left(X_{n}(\mathbb{C}), *\right)$. Hence we have an identification

$$
\widehat{B}_{n} \simeq \widehat{\pi}_{1}\left(X_{n} \times \overline{\mathbb{Q}}, *\right) .
$$

Next assume that $*$ is defined over $\mathbb{Q}$. Then by [G1] IX.Théorème 6.1, we have the homotopy exact sequence of the profinite fundamental group

$$
1 \rightarrow \widehat{\pi}_{1}\left(X_{n} \times \overline{\mathbb{Q}}, *\right) \rightarrow \widehat{\pi}_{1}\left(X_{n}, *\right) \rightarrow \widehat{\pi}_{1}(\operatorname{Spec} \mathbb{Q}, *) \rightarrow 1 .
$$

The last $\widehat{\pi}_{1}(\operatorname{Spec} \mathbb{Q}, *)$ is nothing but the absolute Galois group $G_{\mathbb{Q}}$. A point here is that each basepoint $*$ determines a section $s_{*}$ of the exact sequence. By (1.11), the section $s_{*}$ yields a continuous Galois representation on $\widehat{B}_{n}$

$$
\rho_{n, *}: G_{\mathbb{Q}} \rightarrow \text { Aut } \widehat{B}_{n}
$$

by inner conjugation, i.e., $\rho_{n, *}(\sigma)(b)=s_{*}(\sigma) \cdot b \cdot s_{*}(\sigma)^{-1}\left(\sigma \in G_{\mathbb{Q}}\right.$ and $\left.b \in \widehat{B}_{n}\right)$. A specific (tangential in the sense of Deligne [De] basepoint $t_{n}$ is constructed in [IM], where they showed that the resulting $\rho_{n, t_{n}}$ is equal to our $\rho_{n}$ in (1.10).

Special properties of the $\widehat{G T}$-action in Theorem 1.12 are presented in the following Proposition 1.16 and Proposition 1.18 (though they are implicitly suggested in Dr2]). They will be employed several times in our paper.

Notation 1.15. Put $n>0$ and $m_{1}, m_{2} \geqslant 0$. On the continuous homomorphism

$$
e_{m_{1}} \otimes \cdot \otimes e_{m_{2}}: \widehat{B}_{n} \rightarrow \widehat{B}_{m_{1}+n+m_{2}}
$$

which is defined by $\sigma_{i} \mapsto \sigma_{m_{1}+i}$ (obtained by placing the trivial braids $e_{m_{1}}$ and $e_{m_{2}}$ on the left and right respectively), we denote the image of $b \in \widehat{B}_{n}$ by $e_{m_{1}} \otimes b \otimes e_{m_{2}}$.

Proposition 1.16. Put $n>0$ and $m_{1}, m_{2} \geqslant 0$. Let $\sigma=(\lambda, f) \in \widehat{G T}$ and $b \in \widehat{B}_{n}$. Then

$$
\sigma\left(e_{m_{1}} \otimes b \otimes e_{m_{2}}\right)=f_{\left[m_{1}\right],[n],\left[m_{2}\right]}^{-1} \cdot\left(e_{m_{1}} \otimes \sigma(b) \otimes e_{m_{2}}\right) \cdot f_{\left[m_{1}\right],[n],\left[m_{2}\right]} \cdot
$$


Here

$$
\begin{aligned}
& f_{\left[m_{1}\right],[n],\left[m_{2}\right]}:=f_{1 \cdots m_{1}, m_{1}+1 \cdots m_{1}+n-1, m_{1}+n} . \\
& \quad f_{1 \cdots m_{1}, m_{1}+1 \cdots m_{1}+n-2, m_{1}+n-1} \cdots f_{1 \cdots m_{1}, m_{1}+1, m_{1}+2} \in \widehat{B}_{m_{1}+n+m_{2}} .
\end{aligned}
$$

Proof. It is enough to check (1.12) for $b=\sigma_{i}(1 \leqslant i \leqslant n-1)$. By Theorem 1.12.

$$
\begin{gathered}
f_{\left[m_{1}\right],[n],\left[m_{2}\right]}^{-1} \cdot\left(e_{m_{1}} \otimes \sigma\left(\sigma_{i}\right) \otimes e_{m_{2}}\right) \cdot f_{\left[m_{1}\right],[n],\left[m_{2}\right]}= \\
f_{\left[m_{1}\right],[n],\left[m_{2}\right]}^{-1} \cdot f_{m_{1}+1 \cdots m_{1}+i-1, m_{1}+i, m_{1}+i+1}^{-1} \cdot \sigma_{m_{1}+i}^{\lambda} \cdot f_{m_{1}+1 \cdots m_{1}+i-1, m_{1}+i, m_{1}+i+1} \cdot f_{\left[m_{1}\right],[n],\left[m_{2}\right]} .
\end{gathered}
$$

- When $M \geqslant i+2$, both $f_{m_{1}+1 \cdots m_{1}+i-1, m_{1}+i, m_{1}+i+1}$ and $\sigma_{m_{1}+i}$ commute with $f_{1 \cdots m_{1}, m_{1}+1 \cdots m_{1}+M-1, m_{1}+M}$ because $x_{m_{1}+1 \cdots m_{1}+i-1, m_{1}+i}, x_{m_{1}+i, m_{1}+i+1}$ and $\sigma_{m_{1}+i}$ commute with $x_{1 \cdots m_{1}, m_{1}+1 \cdots m_{1}+M-1}$ and $x_{m_{1}+1 \cdots m_{1}+M-1, m_{1}+M}$. Therefore

$$
\text { (1.14) } \begin{aligned}
& f_{\left[m_{1}\right],[i+1],\left[m_{2}\right]}^{-1} \cdot f_{m_{1}+1 \cdots m_{1}+i-1, m_{1}+i, m_{1}+i+1}^{-1} \cdot \sigma_{m_{1}+i}^{\lambda} \\
& \cdot f_{m_{1}+1 \cdots m_{1}+i-1, m_{1}+i, m_{1}+i+1} \cdot f_{\left[m_{1}\right],[i+1],\left[m_{2}\right]} .
\end{aligned}
$$

- When $M=i, i+1$, our calculation goes as follows.

$$
\begin{aligned}
\text { (1.14) }= & f_{\left[m_{1}\right],[i-1],\left[m_{2}\right]}^{-1} \cdot f_{1 \cdots m_{1}, m_{1}+1 \cdots m_{1}+i-1, m_{1}+i}^{-1} \cdot f_{1 \cdots m_{1}, m_{1}+1 \cdots m_{1}+i, m_{1}+i+1}^{-1} \cdot \\
& f_{m_{1}+1 \cdots m_{1}+i-1, m_{1}+i, m_{1}+i+1}^{-1} \cdot \sigma_{m_{1}+i}^{\lambda} \cdot f_{m_{1}+1 \cdots m_{1}+i-1, m_{1}+i, m_{1}+i+1} \cdot \\
& f_{1 \cdots m_{1}, m_{1}+1 \cdots m_{1}+i, m_{1}+i+1} \cdot f_{1 \cdots m_{1}, m_{1}+1 \cdots m_{1}+i-1, m_{1}+i} \cdot f_{\left[m_{1}\right],[i-1],\left[m_{2}\right]},
\end{aligned}
$$

by the pentagon equation (1.4),

$$
\begin{aligned}
= & f_{\left[m_{1}\right],[i-1],\left[l+m_{2}\right]}^{-1} \cdot f_{1 \cdots m_{1}+i-1, m_{1}+i, m_{1}+i+1}^{-1} . \\
& f_{1 \cdots m_{1}, m_{1}+1 \cdots m_{1}+i-1, m_{1}+i}^{-1} m_{1}+i+1 \\
& \cdot \sigma_{m_{1}+i}^{\lambda} \cdot f_{1 \cdots m_{1}, m_{1}+1 \cdots m_{1}+i-1, m_{1}+i m_{1}+i+1} . \\
& f_{1 \cdots m_{1}+i-1, m_{1}+i, m_{1}+i+1} \cdot f_{\left[m_{1}\right],[i-1],\left[l+m_{2}+2\right]} .
\end{aligned}
$$

Since $\sigma_{m_{1}+i}$ commutes with $f_{1 \cdots m_{1}, m_{1}+1 \cdots m_{1}+i-1, m_{1}+i} m_{1}+i+1$,

$$
\begin{gathered}
=f_{\left[m_{1}\right],[i-1],\left[l+m_{2}\right]}^{-1} \cdot f_{1 \cdots m_{1}+i-1, m_{1}+i, m_{1}+i+1}^{-1} \cdot \sigma_{m_{1}+i}^{\lambda} . \\
f_{1 \cdots m_{1}+i-1, m_{1}+i, m_{1}+i+1} \cdot f_{\left[m_{1}\right],[i-1],\left[l+m_{2}+2\right]} .
\end{gathered}
$$

- When $M \leqslant i-1$, both $f_{1 \cdots m_{1}+i-1, m_{1}+i, m_{1}+i+1}$ and $\sigma_{m_{1}+i}$ commute with $f_{1 \cdots m_{1}, m_{1}+1 \cdots m_{1}+M-1, m_{1}+M}$. Therefore

$$
\begin{aligned}
\text { (1.14) } & =f_{1 \cdots m_{1}+i-1, m_{1}+i, m_{1}+i+1}^{-1} \cdot \sigma_{m_{1}+i}^{\lambda} \cdot f_{1 \cdots m_{1}+i-1, m_{1}+i, m_{1}+i+1} \\
& =\sigma\left(\sigma_{m_{1}+i}\right)=\sigma\left(e_{m_{1}} \otimes \sigma_{i} \otimes e_{m_{2}}\right) .
\end{aligned}
$$

Hence we get the equality (1.12).

Notation 1.17. Let $l, n \geqslant 1$ and $1 \leqslant k \leqslant l$. We consider the continuous group homomorphism

$$
P_{l} \rightarrow P_{l+n-1}
$$


sending, for $1 \leqslant i<j \leqslant l$,

$$
x_{i, j} \mapsto \begin{cases}x_{i+k-1, j+k-1} & (k<i), \\ x_{i \cdots i+k-1, j+k-1} & (k=i), \\ x_{i, j+k-1} & (i<k<j), \\ x_{i, j \cdots j+k-1} & (k=j), \\ x_{i, j} & (j<k) .\end{cases}
$$

We obtain the map by replacing the $k$-th string (from the left) by the trivial braid $e_{n}$ with $n$ strings, hence it naturally extends to two maps (not homomorphisms)

$$
\mathrm{ev}_{k, e_{n}}: B_{l} \rightarrow B_{l+n-1} \quad \text { and } \quad \mathrm{ev}^{k, e_{n}}: B_{l} \rightarrow B_{l+n-1}
$$

which replaces the $k$-th string (from the bottom and the above left respectively) by the trivial braid $e_{n}$ with $n$ strings. Both of their restrictions into $P_{l}$ are equal to the above map (1.15). Since the map (1.15) also continuously extends into the homomorphism $\widehat{P}_{l} \rightarrow \widehat{P}_{l}$, our two maps naturally extend to the maps (not homomorphisms)

$$
\mathrm{ev}_{k, e_{n}}: \widehat{B}_{l} \rightarrow \widehat{B}_{l+n-1} \quad \text { and } \quad \mathrm{ev}^{k, e_{n}}: \widehat{B}_{l} \rightarrow \widehat{B}_{l+n-1} \text {. }
$$

Here we employ the same symbols because there would be no confusion.

Proposition 1.18. Put $l \geqslant 1$. Let $\sigma=(\lambda, f) \in \widehat{G T}$ and $b \in \widehat{B}$. Set $k^{\prime}=b(k)$. Here $b(k)$ stands for the image of $k$ by the permutation corresponding to $b$ by the projection $B_{l} \rightarrow \mathfrak{S}_{l}$. Then, for each $k$ with $1 \leqslant k \leqslant l$, we have

$$
\begin{aligned}
\sigma\left(\operatorname{ev}_{k, e_{n}}(b)\right) & =f_{\left[k^{\prime}-1\right],[n],\left[l-k^{\prime}\right]}^{-1} \cdot \operatorname{ev}_{k, e_{n}}(\sigma(b)) \cdot f_{[k-1],[n],[l-k]} \\
\sigma\left(\operatorname{ev}^{k^{\prime}, e_{n}}(b)\right) & =f_{\left[k^{\prime}-1\right],[n],\left[l-k^{\prime}\right]}^{-1} \cdot \mathrm{ev}^{k^{\prime}, e_{n}}(\sigma(b)) \cdot f_{[k-1],[n],[l-k]}
\end{aligned}
$$

Proof. It suffices to prove the first equality, for the validity of the second equality is immediate once we have the first equality. Firstly we prove (1.16) for $b=\sigma_{i}$ $(1 \leqslant i \leqslant l-1)$.

- When $k<i$, we have $\operatorname{ev}_{k, e_{n}}\left(\sigma_{i}\right)=\sigma_{i+n-1}$ and $k^{\prime}=k$. Therefore

$$
\begin{aligned}
\operatorname{RHS} & =f_{[k-1],[n],[l-k]}^{-1} \cdot \operatorname{ev}_{k, e_{n}}\left(f_{1 \cdots i-1, i, i+1}^{-1} \sigma_{i}^{\lambda} f_{1 \cdots i-1, i, i+1}\right) \cdot f_{[k-1],[n],[l-k]} \\
& =f_{[k-1],[n],[l-k]}^{-1} \cdot\left(f_{1 \cdots i+n-2, i+n-1, i+n}^{-1} \sigma_{i+n-1}^{\lambda} f_{1 \cdots i+n-2, i+n-1, i+n}\right) \cdot f_{[k-1],[n],[l-k]} .
\end{aligned}
$$

By $k-1+n \leqslant i+n-2, f_{[k-1],[n],[l-k]}$ commutes with $f_{1 \cdots i+n-2, i+n-1, i+n}$ and $\sigma_{i+n-1}$. Thus

$$
\begin{aligned}
& =f_{1 \cdots i+n-2, i+n-1, i+n}^{-1} \sigma_{i+n-1}^{\lambda} f_{1 \cdots i+n-2, i+n-1, i+n}=\sigma\left(\sigma_{i+n-1}\right) \\
& =\sigma\left(\operatorname{ev}_{k, e_{n}}\left(\sigma_{i}\right)\right)=\mathrm{LHS} .
\end{aligned}
$$


- When $k=i$, we have $\operatorname{ev}_{k, e_{n}}\left(\sigma_{i}\right)=\operatorname{ev}_{i, e_{n}}\left(\sigma_{i}\right)=\sigma_{i} \sigma_{i+1} \cdots \sigma_{i+n-1}$ and $k^{\prime}=$ $k+1=i+1$. Therefore

$$
\begin{aligned}
\operatorname{RHS}= & f_{[i],[n],[l-i-1]}^{-1} \cdot \mathrm{ev}_{i, e_{n}}\left(f_{1 \cdots i-1, i, i+1}^{-1} \sigma_{i}^{\lambda} f_{1 \cdots i-1, i, i+1}\right) \cdot f_{[i-1],[n],[l-i]} \\
= & f_{[i],[n],[l-i-1]}^{-1} \cdot f_{1 \cdots i-1, i, i+1 \cdots i+n}^{-1} \cdot \mathrm{ev}_{i, e_{n}}\left(\sigma_{i}^{\lambda}\right) \cdot f_{1 \cdots i-1, i \cdots i+n-1, i+n} \cdot f_{[i-1],[n],[l-i]} \\
= & f_{[i],[n],[l-i-1]}^{-1} \cdot f_{1 \cdots i-1, i, i+1 \cdots i+n}^{-1} \cdot \mathrm{ev}_{i, e_{n}}\left(\sigma_{i} \cdot x_{i, i+1}^{m}\right) \cdot f_{[i-1],[n+1],[l-i-1]} \\
= & f_{[i],[n],[l-i-1]}^{-1} \cdot f_{1 \cdots i-1, i, i+1 \cdots i+n}^{-1} \cdot\left(\sigma_{i} \cdots \sigma_{i+n-1}\right) \cdot x_{i \cdots i+n-1, i+n}^{m} \\
& \quad \cdot f_{[i-1],[n+1],[l-i-1]} \\
= & f_{[i],[n],[l-i-1]}^{-1} \cdot f_{1 \cdots i-1, i, i+1 \cdots i+n}^{-1} \cdot\left(e_{i-1} \otimes\left(\sigma_{1} \cdots \sigma_{n}\right) \cdot x_{1 \cdots n, n+1}^{m} \otimes e_{l-i-1}\right) \\
& \quad f_{[i-1],[n+1],[l-i-1]} .
\end{aligned}
$$

By (1.9),

$$
\begin{gathered}
=f_{[i],[n],[l-i-1]}^{-1} \cdot f_{1 \cdots i-1, i, i+1 \cdots i+n}^{-1} \cdot\left(e_{i-1} \otimes f_{[1],[n],[0]} \cdot \sigma\left(\sigma_{1} \cdots \sigma_{n}\right) \otimes e_{l-i-1}\right) \\
\cdot f_{[i-1],[n+1],[l-i-1]} \\
=f_{[i],[n],[l-i-1]}^{-1} \cdot f_{1 \cdots i-1, i, i+1 \cdots i+n}^{-1} \cdot\left(e_{i-1} \otimes f_{[1],[n],[0]} \cdot \otimes e_{l-i-1}\right) \\
\cdot\left(e_{i-1} \otimes \sigma\left(\sigma_{1} \cdots \sigma_{n}\right) \otimes e_{l-i-1}\right) \cdot f_{[i-1],[n+1],[l-i-1]} \cdot
\end{gathered}
$$

By Lemma 1.19 below,

$=f_{[i-1],[n+1],[l-i-1]}^{-1} \cdot\left(e_{i-1} \otimes \sigma\left(\sigma_{1} \cdots \sigma_{n}\right) \otimes e_{l-i-1}\right) \cdot f_{[i-1],[n+1],[l-i-1]} \cdot$

\section{By Proposition 1.16,}

$=\sigma\left(e_{i-1} \otimes\left(\sigma_{1} \cdots \sigma_{n}\right) \otimes e_{l-i-1}\right)=\sigma\left(\sigma_{i} \cdots \sigma_{i+n-1}\right)=\sigma\left(\mathrm{ev}_{i, e_{n}}\left(\sigma_{i}\right)\right)=$ LHS.

- When $k=i+1$, we have $\operatorname{ev}_{k, e_{n}}\left(\sigma_{i}\right)=\operatorname{ev}_{i+1, e_{n}}\left(\sigma_{i}\right)=\sigma_{i+n-1} \cdots \sigma_{i+1} \sigma_{i}$ and $k^{\prime}=k-1=i$. Therefore

$$
\begin{aligned}
\operatorname{RHS}= & f_{[i-1],[n],[l-i]}^{-1} \cdot \mathrm{ev}_{i+1, e_{n}}\left(f_{1 \cdots i-1, i, i+1}^{-1} \sigma_{i}^{\lambda} f_{1 \cdots i-1, i, i+1}\right) \cdot f_{[i],[n],[l-i-1]} \\
= & f_{[i-1],[n],[l-i]}^{-1} \cdot f_{1 \cdots i-1, i \cdots i+n-1, i+n}^{-1} \cdot \mathrm{ev}_{i+1, e_{n}}\left(\sigma_{i}^{\lambda}\right) \cdot f_{1 \cdots i-1, i, i+1 \cdots i+n} \cdot f_{[i],[n],[l-i-1]} \\
= & f_{[i-1],[n+1],[l-i-1]}^{-1} \cdot \mathrm{ev}_{i+1, e_{n}}\left(x_{i, i+1}^{m} \cdot \sigma_{i}\right) \cdot f_{1 \cdots i-1, i, i+1 \cdots i+n} \cdot f_{[i],[n],[l-i-1]} \\
= & f_{[i-1],[n+1],[l-i-1]}^{-1} \cdot x_{i \cdots i+n-1, i+n}^{m} \cdot\left(\sigma_{i+n-1} \cdots \sigma_{i+1} \sigma_{i}\right) \\
& \quad \cdot f_{1 \cdots i-1, i, i+1 \cdots i+n} \cdot f_{[i],[n],[l-i-1]} \\
= & f_{[i-1],[n+1],[l-i-1]}^{-1} \cdot\left(e_{i-1} \otimes x_{1 \cdots n, n+1}^{m} \cdot\left(\sigma_{n} \cdots \sigma_{1}\right) \otimes e_{l-i-1}\right) \\
& \quad \cdot f_{1 \cdots i-1, i, i+1 \cdots i+n} \cdot f_{[i],[n],[l-i-1]} \cdot
\end{aligned}
$$

By (1.9),

$$
\begin{aligned}
& =f_{[i-1],[n+1],[l-i-1]}^{-1} \cdot\left(e_{i-1} \otimes \sigma\left(\sigma_{n} \cdots \sigma_{1}\right) \cdot f_{[1],[n],[0]}^{-1} \otimes e_{l-i-1}\right) \\
& \quad \cdot f_{1 \cdots i-1, i, i+1 \cdots i+n} \cdot f_{[i],[n],[l-i-1]} \\
& =f_{[i-1],[n+1],[l-i-1]}^{-1} \cdot\left(e_{i-1} \otimes \sigma\left(\sigma_{n} \cdots \sigma_{1}\right) \otimes e_{l-i-1}\right) \cdot\left(e_{i-1} \otimes f_{[1],[n],[0]}^{-1} \cdot \otimes e_{l-i-1}\right) \\
& \quad \cdot f_{1 \cdots i-1, i, i+1 \cdots i+n} \cdot f_{[i],[n],[l-i-1]} \cdot
\end{aligned}
$$


By Lemma 1.19 below,

$=f_{[i-1],[n+1],[l-i-1]}^{-1} \cdot\left(e_{i-1} \otimes \sigma\left(\sigma_{n} \cdots \sigma_{1}\right) \otimes e_{l-i-1}\right) \cdot f_{[i-1],[n+1],[l-i-1]} \cdot$

By Proposition 1.16,

$=\sigma\left(e_{i-1} \otimes\left(\sigma_{n} \cdots \sigma_{1}\right) \otimes e_{l-i-1}\right)=\sigma\left(\sigma_{i+n-1} \cdots \sigma_{i}\right)=\sigma\left(\operatorname{ev}_{i+1, e_{n}}\left(\sigma_{i}\right)\right)=$ LHS.

- When $k>i+1$, we have $\mathrm{ev}_{k, e_{n}}\left(\sigma_{i}\right)=\sigma_{i}$ and $k^{\prime}=k$. Therefore

$$
\begin{aligned}
\operatorname{RHS} & =f_{[k-1],[n],[l-k]}^{-1} \cdot \operatorname{ev}_{k, e_{n}}\left(f_{1 \cdots i-1, i, i+1}^{-1} \sigma_{i}^{\lambda} f_{1 \cdots i-1, i, i+1}\right) \cdot f_{[k-1],[n],[l-k]} \\
& =f_{[k-1],[n],[l-k]}^{-1} \cdot\left(f_{1 \cdots i-1, i, i+1}^{-1} \sigma_{i}^{\lambda} f_{1 \cdots i-1, i, i+1}\right) \cdot f_{[k-1],[n],[l-k]} .
\end{aligned}
$$

By $i+1 \leqslant k-1, f_{[k-1],[n],[l-k]}$ commutes with $f_{1 \cdots i-1, i, i+1}$ and $\sigma_{i}$. Thus

$$
=f_{1 \cdots i-1, i, i+1}^{-1} \sigma_{i}^{\lambda} f_{1 \cdots i-1, i, i+1}=\sigma\left(\sigma_{i}\right)=\sigma\left(\operatorname{ev}_{k, e_{n}}\left(\sigma_{i}\right)\right)=\text { LHS. }
$$

Whence the equation (1.16) for $b=\sigma_{i}$ is obtained.

The validity for $b=\sigma_{i}$ implies the validity for $b \in B_{l}$ because each element of $B_{l}$ is a finite product of $\sigma_{i}$ 's. Whence particularly we have the validity for $P_{l}$. Then by continuity we have for $\widehat{P}_{l}$. Since we have the validity for $B_{l}$ and $\widehat{P}_{l}$, we have the validity for $\widehat{B}_{l}$.

The auxiliary lemma below is required to prove the above proposition.

Lemma 1.19. For $\sigma=(\lambda, f) \in \widehat{G T}$ and $i, n, l>0$ with $l>i$, the following equation holds in $\widehat{B}_{l+n-1}$ :

$$
\left(e_{i-1} \otimes f_{[1],[n],[0]} \otimes e_{l-i-1}\right) \cdot f_{[i-1],[n+1],[l-i-1]}=f_{1 \cdots i-1, i, i+1 \cdots i+n} \cdot f_{[i],[n],[l-i-1]} .
$$

Proof. The above equation can be read as

$$
\begin{array}{r}
\left(f_{i, i+1 \cdots i+n-1, i+n} \cdots f_{i, i+1, i+2}\right) \cdot f_{1 \cdots i-1, i \cdots i+n-1, i+n} \cdots f_{1 \cdots i-1, i, i+1} \\
=f_{1 \cdots i-1, i, i+1 \cdots i+n} \cdot\left(f_{1 \cdots i, i+1 \cdots i+n-1, i+n} \cdots f_{1 \cdots i, i+1, i+2}\right) .
\end{array}
$$

It can be proved by successive applications of (1.4).

\section{Profinite Knots}

This section is to present our main results. Our ABC-construction of profinite knots is introduced and the basic properties of profinite knots are shown in $\$ 2.1$. An action of the absolute Galois group on profinite knots is rigorously established in 42.3. where the notion of pro- $l$ knots introduced in $\$ 2.2$ serves to show its property.

2.1. ABC-construction. Profinite tangle diagrams, profinite analogues of tangle diagrams, are introduced as consistent finite sequences of symbols of three types $A$, $\widehat{B}$ and $C$ in Definition 2.3. Profinite link diagrams mean profinite tangle diagrams without endpoints and profinite knot diagrams mean profinite link diagrams with a single connected component (Definition 2.4). The notion of isotopy for them are given by a profinite analogue of Turaev moves in Definition 2.8. Two fundamental properties for the set $\widehat{\mathcal{K}}$ of profinite knots (isotopy classes of profinite knot diagrams) are presented; Theorem 2.11 explains that there is a natural map from the set $\mathcal{K}$ of isotopy classes of (topological) knots to our set $\widehat{\mathcal{K}}$ and our Theorem 2.16] says that our $\widehat{\mathcal{K}}$ carries a structure of a topological commutative monoid.

Here is a brief review of tangles and knots. 
HIDEKAZU FURUSHO

Notation 2.1. Let $k, l \geqslant 0$. Let $\epsilon=\left(\epsilon_{1}, \ldots, \epsilon_{k}\right)$ and $\epsilon^{\prime}=\left(\epsilon_{1}^{\prime}, \ldots, \epsilon_{1}^{\prime}\right)$ be sequences (including the empty sequence $\emptyset$ ) of symbols $\uparrow$ and $\downarrow$. An (oriented) $3^{3}$ tangle of type $\left(\epsilon, \epsilon^{\prime}\right)$ means a smooth embedded compact oriented one-dimensional real manifolds in $[0,1] \times \mathbb{C}$ (hence it is a finite disjoint union of embedded one-dimensional intervals and circles), whose boundaries are $\{(1,1), \ldots,(1, k),(0,1) \ldots,(0, l)\}$ such that $\epsilon_{i}$ (resp. $\epsilon_{j}^{\prime}$ ) is $\uparrow$ or $\downarrow$ if the tangle is oriented upwards or downwards at $(1, i)$ (resp. at $(0, j))$ respectively. A link is a tangle of type $(\emptyset, \emptyset)$, i.e. $k=l=0$, and a knot means a link with a single connected component.

A tangle is like 'a braid' whose each connected component is allowed to be a circle and have endpoints on the same plane. Figure 2.1 might help the readers to have a good understanding of the definition.

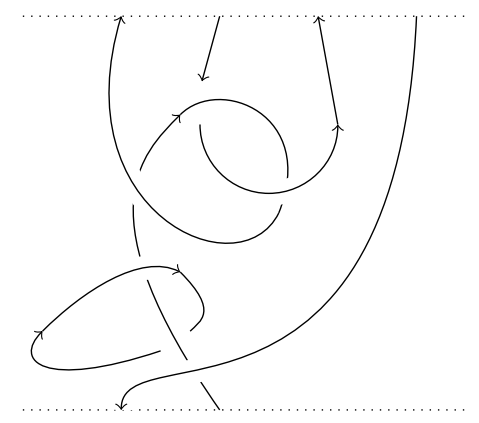

Figure 2.1. A tangle of type $(\uparrow \downarrow \uparrow \downarrow, \downarrow \uparrow)$

The following notion of profinite fundamental tangle diagrams plays a role of composite elements of the notion of profinite tangles.

Definition 2.2. The set of fundamental profinite tangle diagrams means the disjoint union of the following three sets $A, \widehat{B}$ and $C$ 田 of symbols:

$$
\begin{aligned}
& A:=\left\{a_{k, l}^{\epsilon} \mid k, l=0,1,2, \ldots, \epsilon=\left(\epsilon_{i}\right)_{i=1}^{k+l+1} \in\{\uparrow, \downarrow\}^{k} \times\{\curvearrowright, \curvearrowleft\} \times\{\uparrow, \downarrow\}^{l}\right\}, \\
& \widehat{B}:=\left\{b_{n}^{\epsilon} \mid b_{n}^{\epsilon}=\left(b_{n}, \epsilon=\left(\epsilon_{i}\right)_{i=1}^{n}\right) \in \widehat{B}_{n} \times\{\uparrow, \downarrow\}^{n}, n=1,2,3,4, \ldots\right\}, \\
& C:=\left\{c_{k, l}^{\epsilon} \mid k, l=0,1,2, \ldots, \epsilon=\left(\epsilon_{i}\right)_{i=1}^{k+l+1} \in\{\uparrow, \downarrow\}^{k} \times\{\cup, \smile\} \times\{\uparrow, \downarrow\}^{l}\right\} .
\end{aligned}
$$

Here all arrows are merely regarded as symbols.

We occasionally depict these fundamental profinite tangle diagrams with ignorance of arrows (which represent orientation of each strings) as the pictures in Figure 2.2 which we call their topological pictures.

For a fundamental profinite tangle diagram $\gamma$, its source $s(\gamma)$ and its target $t(\gamma)$ are sequences of $\uparrow$ and $\downarrow$ defined below:

(1) When $\gamma=a_{k, l}^{\epsilon}, s(\gamma)$ is the sequence of $\uparrow$ and $\downarrow$ replacing $\curvearrowright$ (resp. $\curvearrowleft$ ) by $\uparrow \downarrow$ (resp. $\downarrow \uparrow$ ) in $\epsilon$ and $t(\gamma)$ is the sequence omitting $\curvearrowright$ and $\curvearrowleft$ in $\epsilon$ (cf. Figure2.3).

\footnotetext{
${ }^{3}$ We occasionally omit to mention it. Throughout the paper all tangles are assumed to be oriented.

${ }^{4}$ A, B and C stand for Annihilations, Braids and Creations respectively.
} 

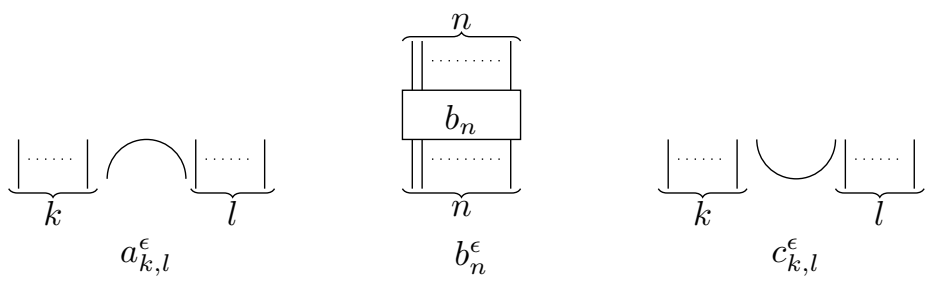

FIgURE 2.2. Topological picture of fundamental profinite tangle diagrams

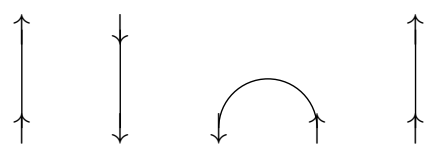

Figure 2.3. $a_{2,1}^{\epsilon}$ with $s\left(a_{2,1}^{\epsilon}\right)=\uparrow \downarrow \downarrow \uparrow \uparrow$ and $t\left(a_{2,1}^{\epsilon}\right)=\uparrow \downarrow \uparrow$

(2) When $\gamma=b_{n}^{\epsilon}, s(\gamma)=\epsilon$ and $t(\gamma)$ is the permutation of $\epsilon$ induced by the image of $b_{n}^{\epsilon}$ of the projection $\widehat{B}_{n}$ to the symmetric group $\mathfrak{S}_{n}$ (cf. Figure 2.4).

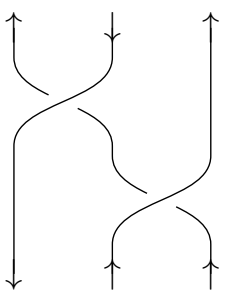

FiguRE 2.4. An example of $b_{3}^{\epsilon}$ with $s\left(b_{3}^{\epsilon}\right)=\epsilon=\downarrow \uparrow \uparrow$ and $t\left(b_{3}^{\epsilon}\right)=\uparrow \downarrow \uparrow$

(3) When $\gamma=c_{k, l}^{\epsilon}, s(\gamma)$ is the set omitting $\checkmark$ and $\smile$ in $\epsilon$ and $t(\gamma)$ is the set replacing $\cup($ resp. $\cup$ ) by $\downarrow \uparrow($ resp. $\uparrow \downarrow)$ in $\epsilon$.

Definition 2.3. A profinite (oriented) tangle diagram means a finite consistent 5 sequence $T=\left\{\gamma_{i}\right\}_{i=1}^{n}$ of fundamental profinite tangles (which we denote by $\left.\gamma_{n} \cdots \gamma_{2} \cdot \gamma_{1}\right)$. Its source and its target are defined by $s(T):=s\left(\gamma_{1}\right)$ and $t(T):=$ $t\left(\gamma_{n}\right)$. A profinite (oriented) link diagram means a profinite tangle diagram $T$ with $s(T)=t(T)=\emptyset$.

For a fundamental profinite tangle diagram $\gamma$, its skeleton $\mathbb{S}(\gamma)$ is the graph consisting of finitely many vertices and finitely many edges connecting them as follows:

(1) When $\gamma=a_{k, l}^{\epsilon}$ or $c_{k, l}^{\epsilon}, \mathbb{S}(\gamma)$ is nothing but the graph of the topological picture of $\gamma$ in Figure 2.2 whose set of vertices is the collection of its endpoints

\footnotetext{
${ }^{5}$ Here 'consistent' means successively composable, that is, $s\left(\gamma_{i+1}\right)=t\left(\gamma_{i}\right)$ holds for all $i=$ $1,2, \ldots, n-1$.
} 
and whose set of edges is given by the arrows connecting them (cf. Figure 2.5).

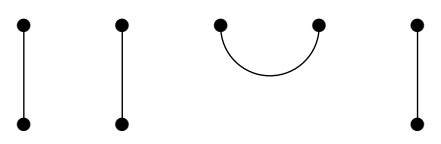

Figure 2.5. Skeleton of Figure 2.3

(2) When $\gamma=b_{n}^{\epsilon}, \mathbb{S}(\gamma)$ is the graph describing the permutation $p\left(b_{n}^{\epsilon}\right)$ (cf. Figure [2.6), where $p$ means the projection $\widehat{B}_{n} \rightarrow \mathfrak{S}_{n}$. Namely its set of vertices is the collection of its endpoints and its set of edges is the set of diagonal lines combining the corresponding vertices.

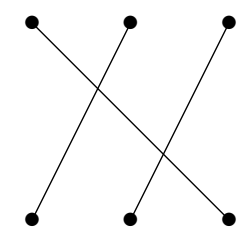

FigurE 2.6. Skeleton of Figure 2.4

For a profinite tangle diagram $T=\gamma_{n} \cdots \gamma_{2} \cdot \gamma_{1}$, its skeleton $\mathbb{S}(T)=\mathbb{S}\left(\gamma_{n}\right) \cdots \mathbb{S}\left(\gamma_{2}\right)$. $\mathbb{S}\left(\gamma_{1}\right)$ means the graph obtained by the composition of $\mathbb{S}\left(\gamma_{i}\right)(1 \leqslant i \leqslant n)$ (cf. Figure 2.7 2.8).

Definition 2.4. A connected component of a profinite tangle diagram $T$ means a connected component of the skeleton $\mathbb{S}(T)$ as a graph. A profinite (oriented) knot diagram means a profinite (oriented) link diagram with a single connected component.

It is easy to see that the number of connected components of any profinite tangle diagram is always finite. A profinite knot diagram is a profinite version of a (topological) oriented knot diagram.

Problem 2.5. Can we regard a profinite knot as a wild knot6?

It might be nice if we could give any topological meaning for all (or a part of) profinite knot diagrams.

Example 2.6. The profinite link diagram

$$
a_{0,0}^{\curvearrowleft} \cdot a_{2,0}^{\downarrow \uparrow \curvearrowright} \cdot\left(\sigma_{2}^{\downarrow \uparrow \uparrow \downarrow}\right)^{\lambda} \cdot c_{2,0}^{\downarrow \uparrow \cup} \cdot c_{0,0}^{\hookrightarrow} \quad(\lambda \in \widehat{\mathbb{Z}})
$$

depicted in Figure 2.7 (here $\sigma_{2}^{\downarrow \uparrow \uparrow \downarrow}$ is the generator $\sigma_{2}$ of $\widehat{B_{4}}$ ) is with 2 connected components if $\lambda \equiv 0(\bmod 2)$ and is with a single connected component (hence is a profinite knot) if $\lambda \equiv 1(\bmod 2)$. (cf. Figure 2.8$)$.

\footnotetext{
${ }^{6}$ A wild knot means a topological embedding of the oriented circle into $S^{3}$ (or $\mathbb{R}^{3}$ ).
} 


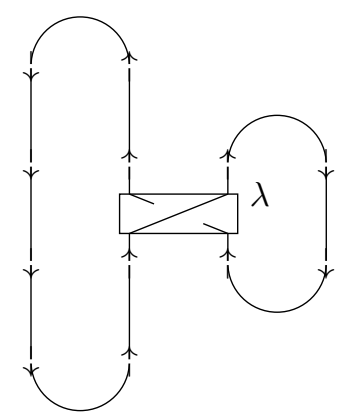

FIGURE 2.7. Is this a profinite knot?

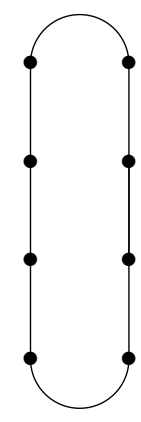

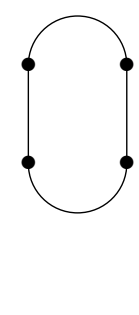

$\lambda \equiv 0(\bmod 2)$

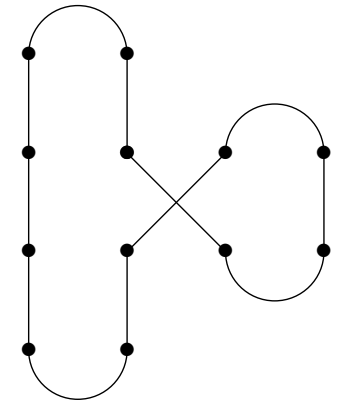

$\lambda \equiv 1(\bmod 2)$

Figure 2.8. Skeleton of Figure 2.7

Notation 2.7. (1) The symbol $e_{n}^{\epsilon}=\left(e_{n}, \epsilon\right)$ stands for the fundamental profinite tangle diagram in $\widehat{B}$ with $s\left(e_{n}^{\epsilon}\right)=\epsilon$ which corresponds to the trivial braid $e_{n}$ in $\hat{B}_{n}$. For a fundamental profinite tangle diagram $\gamma$, we mean $e_{n_{1}}^{\epsilon_{1}} \otimes \gamma \otimes e_{n_{2}}^{\epsilon_{2}}$ by the fundamental profinite tangle diagram obtained by putting $e_{n_{1}}^{\epsilon_{1}}$ and $e_{n_{2}}^{\epsilon_{2}}$ on the left and on the right of $\gamma$ respectively. So, $e_{n_{1}}^{\epsilon_{1}} \otimes a_{k, l}^{\epsilon} \otimes e_{n_{2}}^{\epsilon_{2}}=a_{n_{1}+k, l+n_{2}}^{\epsilon_{1}, \epsilon, \epsilon_{2}}$, for instance. For a profinite tangle $T=\left\{\gamma_{i}\right\}_{i=1}^{n}$ ( $\gamma_{i}$ : a fundamental profinite tangle diagram), $e_{n_{1}}^{\epsilon_{1}} \otimes T \otimes e_{n_{2}}^{\epsilon_{2}}$ means the profinite tangle diagram $\left\{e_{n_{1}}^{\epsilon_{1}} \otimes \gamma_{i} \otimes e_{n_{2}}^{\epsilon_{2}}\right\}_{i=1}^{n}$.

(2) Let $\epsilon_{0} \in\{\uparrow, \downarrow\}^{n}$ for some $n$. For a fundamental profinite tangle diagram $b_{l}^{\epsilon} \in \widehat{B}$ and $k$ with $1 \leqslant k \leqslant l$, the symbol $\operatorname{ev}_{k, \epsilon_{0}}\left(b_{l}^{\epsilon}\right)\left(\operatorname{resp.} \operatorname{ev}^{k, \epsilon_{0}}\left(b_{l}^{\epsilon}\right)\right)$ means the element in $\widehat{B}$ which represents the profinite braid replacing the $k$-th string (from its bottom (resp. above) left) of $b_{l}^{\epsilon}$ by the trivial braid $e_{n}^{\epsilon_{0}}$ with $n$ strings whose source is $\epsilon_{0}$ (cf. Notation 1.17). For instance, the profinite tangle diagram in Figure 2.4 is described as $\operatorname{ev}_{1, \downarrow \uparrow}(\%)$ or $\mathrm{ev}^{2, \downarrow \uparrow}(\%)$.

Definition 2.8. For profinite tangle diagrams, the moves (T1)-(T6) are defined as follow. 
(T1) Trivial braids invariance: for a profinite tangle diagram $T$ with $|s(T)|=m$ (resp. $|t(T)|=n)$, 写

$$
e_{n}^{t(T)} \cdot T=T=T \cdot e_{m}^{s(T)}
$$

For $e_{n}$, see Notation 2.7 Figure 2.9 depicts the move.

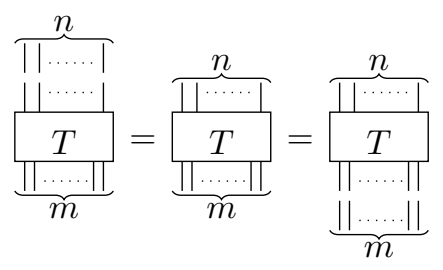

Figure 2.9. (T1): Trivial braids invariance

(T2) Braids composition: for $b_{n}^{\epsilon_{1}}, b_{n}^{\epsilon_{2}} \in \widehat{B}$ with $t\left(b_{n}^{\epsilon_{1}}\right)=s\left(b_{n}^{\epsilon_{2}}\right)$,

$$
b_{n}^{\epsilon_{2}} \cdot b_{n}^{\epsilon_{1}}=b_{n}^{\epsilon_{3}} .
$$

Here $b_{n}^{\epsilon_{3}}$ means the element in $\widehat{B}$ with $s\left(b_{n}^{\epsilon_{3}}\right)=s\left(b_{n}^{\epsilon_{1}}\right)$ and $t\left(b_{n}^{\epsilon_{3}}\right)=t\left(b_{n}^{\epsilon_{2}}\right)$ which represents the product $b_{2} \cdot b_{1}$ of two braids in $\widehat{B}_{n}$. Figure 2.10 depicts the move.

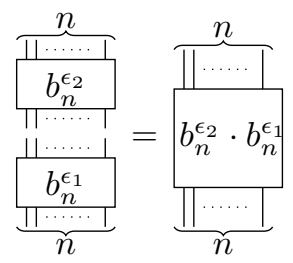

FiguRE 2.10. (T2): Braids composition

(T3) Independent tangles relation: for profinite tangle diagrams $T_{1}$ and $T_{2}$ with $\left|s\left(T_{1}\right)\right|=m_{1},\left|t\left(T_{1}\right)\right|=n_{1},\left|s\left(T_{2}\right)\right|=m_{2}$ and $\left|t\left(T_{2}\right)\right|=n_{2}$,

$$
\left(e_{n_{1}}^{t\left(T_{1}\right)} \otimes T_{2}\right) \cdot\left(T_{1} \otimes e_{m_{2}}^{s\left(T_{2}\right)}\right)=\left(T_{1} \otimes e_{n_{2}}^{t\left(T_{2}\right)}\right) \cdot\left(e_{m_{1}}^{s\left(T_{1}\right)} \otimes T_{2}\right) .
$$

We occasionally denote both hands side by $T_{1} \otimes T_{2}$. For the symbol $\otimes$, see Notation 2.7. Figure 2.11 depicts the move.

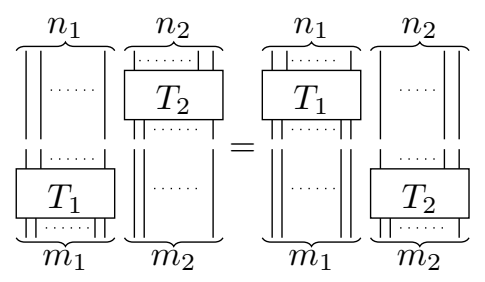

Figure 2.11. (T3): Independent tangles relation

\footnotetext{
${ }^{7}$ For a set $S,|S|$ stands for its cardinality.
} 
(T4) Braid-tangle relations: for $b_{l}^{\epsilon} \in \widehat{B}, k$ with $1 \leqslant k \leqslant l$ and a profinite tangle diagram $T$ with $|s(T)|=m$ and $|t(T)|=n$,

$$
\mathrm{ev}_{k, t(T)}\left(b_{l}^{\epsilon}\right) \cdot\left(e_{k-1}^{s_{1}} \otimes T \otimes e_{l-k}^{s_{2}}\right)=\left(e_{k^{\prime}-1}^{t_{1}} \otimes T \otimes e_{l-k^{\prime}}^{t_{2}}\right) \cdot \mathrm{ev}^{k^{\prime}, s(T)}\left(b_{l}^{\epsilon}\right) .
$$

For ev, see Notation 2.7. For $s\left(b_{l}^{\epsilon}\right)=\epsilon=\left(\epsilon_{i}\right)_{i=1}^{l}$ we put $s_{1}:=\left(\epsilon_{i}\right)_{i=1}^{k-1}$ and $s_{2}:=$ $\left(\epsilon_{i}\right)_{i=k+1}^{l}$. Put $k^{\prime}=b_{l}^{\epsilon}(k)$. Here $b_{l}^{\epsilon}(k)$ stands for the image of $k$ by the permutation which corresponds to $b_{l}^{\epsilon}$ by the projection $B_{l} \rightarrow \mathfrak{S}_{l}$. For $t\left(b_{l}^{\epsilon}\right)=\left(\epsilon_{i}^{\prime}\right)_{i=1}^{l}$ we put $t_{1}:=\left(\epsilon_{i}^{\prime}\right)_{i=1}^{k^{\prime}-1}$ and $t_{2}:=\left(\epsilon_{i}^{\prime}\right)_{i=k^{\prime}+1}^{l}$. Figure 2.12 depicts the move.

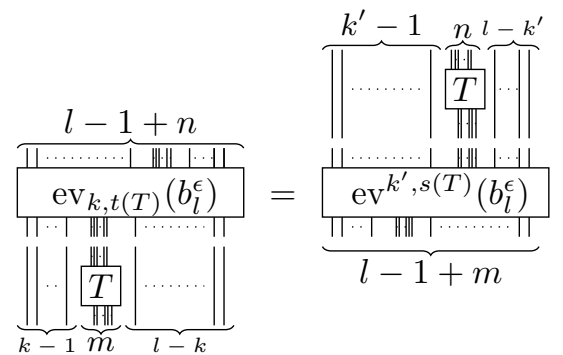

Figure 2.12. (T4): Braid-tangle relation

(T5) Creation-annihilation relation: for $c_{k, l}^{\epsilon} \in C$ and $a_{k+1, l-1}^{\epsilon^{\prime}} \in A$ with $t\left(c_{k, l}^{\epsilon}\right)=$ $s\left(a_{k+1, l-1}^{\epsilon^{\prime}}\right)$

$$
a_{k+1, l-1}^{\epsilon^{\prime}} \cdot c_{k, l}^{\epsilon}=e_{k+l}^{s\left(c_{k, l}^{\epsilon}\right)} .
$$

And for $c_{k, l}^{\epsilon} \in C$ and $a_{k-1, l+1}^{\epsilon^{\prime}} \in A$ with $t\left(c_{k, l}^{\epsilon}\right)=s\left(a_{k-1, l+1}^{\epsilon^{\prime}}\right)$

$$
a_{k-1, l+1}^{\epsilon^{\prime}} \cdot c_{k, l}^{\epsilon}=e_{k+l}^{s\left(c_{k, l}^{\epsilon}\right)} .
$$

Figure 2.13 depicts the move.
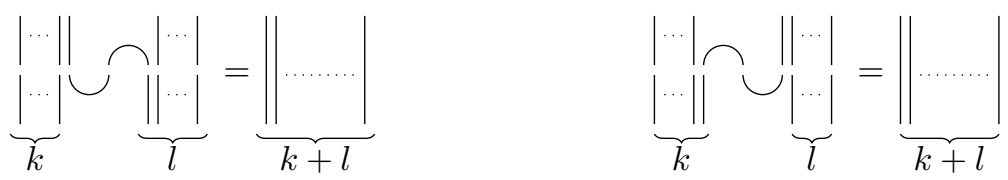

Figure 2.13. (T5): Creation-annihilation relations

(T6) First Reidemeister move: for $c \in \widehat{\mathbb{Z}} \mathbf{8}, c_{k, l}^{\epsilon} \in C$ and $\sigma_{k+1}^{\epsilon^{\prime}} \in \widehat{B}$ which represents $\sigma_{k+1} \in \widehat{B}_{k+l+2}$ and $t\left(c_{k, l}^{\epsilon}\right)=s\left(\left(\sigma_{k+1}^{\epsilon^{\prime}}\right)^{c}\right)$

$$
\left(\sigma_{k+1}^{\epsilon^{\prime}}\right)^{c} \cdot c_{k, l}^{\epsilon}=c_{k, l}^{\bar{\epsilon}}
$$

where $\bar{\epsilon}$ is chosen to be $t(\bar{\epsilon})=t\left(\left(\sigma_{k+1}^{\epsilon^{\prime}}\right)^{c}\right)$.

For $c \in \widehat{\mathbb{Z}}, a_{k, l}^{\epsilon} \in A$ and $\sigma_{k+1}^{\epsilon^{\prime}} \in \widehat{B}$ which represents $\sigma_{k+1} \in \widehat{B}_{k+l+2}$ and $s\left(a_{k, l}^{\epsilon}\right)=$ $t\left(\left(\sigma_{k+1}^{\epsilon^{\prime}}\right)^{c}\right)$

$$
a_{k, l}^{\epsilon} \cdot\left(\sigma_{k+1}^{\epsilon^{\prime}}\right)^{c}=a_{k, l}^{\bar{\epsilon}} .
$$



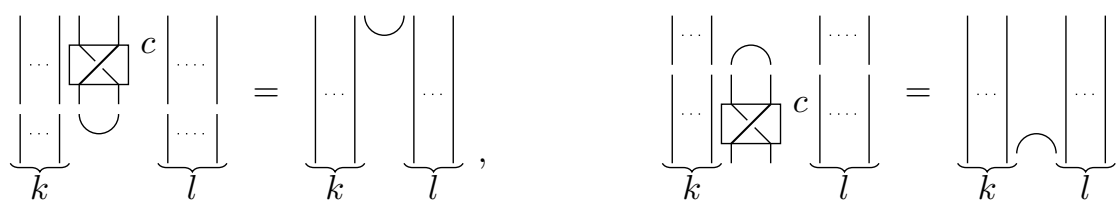

Figure 2.14. (T6): First Reidemeister move

where $\bar{\epsilon}$ is chosen to be $s(\bar{\epsilon})=s\left(\left(\sigma_{k+1}^{\epsilon^{\prime}}\right)^{c}\right)$. Figure 2.14 depicts the moves.

We note that in the first (resp. second) equation $c_{k, l}^{\epsilon}=c_{k, l}^{\bar{\epsilon}}$ (resp. $\left.a_{k, l}^{\epsilon}=a_{k, l}^{\bar{\epsilon}}\right)$ if and only if $c \equiv 0(\bmod 2)$.

These moves (T1)-(T6) are profinite analogues of the so-called Turaev moves $\mathrm{Tu}$. for oriented tangles (consult also [CDM, K, O1]). Our above formulation is stimulated by the moves presented (R1)-(R11) in [Ba1].

Definition 2.9. Two profinite (oriented) tangle diagrams $T_{1}$ and $T_{2}$ are isotopic, denoted $T_{1}=T_{2}$ by abuse of notation, if they are related by a finite number of the moves (T1)-(T6). A profinite tangle, profinite link and profinite knot means an isotopy class of profinite tangle diagrams, link diagrams, knot diagrams respectively. The set of profinite tangles is denoted by $\widehat{\mathcal{T}}$. The set $\widehat{\mathcal{L}}$ of profinite links (resp. the set $\widehat{\mathcal{K}}$ of profinite knots) is the subset of $\widehat{\mathcal{T}}$ which consists of isotopy classes of profinite links (resp. of profinite knots).

Note 2.10. Profinite topology on $\widehat{B}_{n}(n=1,2, \ldots)$ and the discrete topology on $A$ and on $C$ yield a topology on the space of profinite tangles. Hence $\widehat{\mathcal{T}}$ carries a structure of topological space.

Theorem 2.11. (1). Let $\mathcal{T}$ be the set of isotopy classes of (topological) oriented tangles. There is a natural map

$$
h: \mathcal{T} \rightarrow \widehat{\mathcal{T}}
$$

which we call the profinite realization map.

(2). The above profinite realization map induces the map

$$
h: \mathcal{K} \rightarrow \widehat{\mathcal{K}} .
$$

Here $\mathcal{K}$ stands for the set of isotopy classes of topological oriented knots.

Proof. (1). The result in [Ba1] indicates that the set $\mathcal{T}$ is described by the set of consistent finite sequences of fundamental tangles, elements of $A$,

$$
B:=\left\{b_{n}^{\epsilon} \mid b_{n}^{\epsilon}=\left(b_{n}, \epsilon=\left(\epsilon_{i}\right)_{i=1}^{n}\right) \in B_{n} \times\{\uparrow, \downarrow\}^{n}, n=1,2,3,4, \ldots\right\}
$$

and $C$, modulo the (discrete) Turaev moves. Since the (discrete) Turaev moves in this case mean the moves replacing profinite tangles and braids by (discrete) tangles and braids in (T1)-(T6) and $c \in \widehat{\mathbb{Z}}$ by $c \in \mathbb{Z}$ in (T6). Because we have a natural map $B_{n} \rightarrow \widehat{B}_{n}$ and the Turaev moves are special case of our 6 moves, we have a natural map $h: \mathcal{T} \rightarrow \widehat{\mathcal{T}}$.

(2). It is easy because the set of profinite tangles isotopic to a given profinite knot consists of only profinite knots.

\footnotetext{
${ }^{8}$ It should be worthy to emphasize that $c$ is assumed to be not in $\mathbb{Z}$ but in $\widehat{\mathbb{Z}}$.
} 
We notice that the number of connected components is an isotopic invariant of profinite tangles. As a knot analogue of residually-finiteness (1.1) of the braid group $B_{n}$, we raise the conjecture below.

Conjecture 2.12. The map $h: \mathcal{K} \rightarrow \widehat{\mathcal{K}}$ is injective.

Remark 2.13. The Kontsevich invariant is an invariant of oriented knots which is conjectured to be a perfect invariant, i.e. an invariant detecting all oriented knots (cf. O2 etc). We note that if the invariant is perfect, then the map $h$ is injective by the arguments given in Remark 2.34 below.

Since last century, lots of knot invariants have been investigated; such as the unknotting number, the Alexander polynomial, the Jones polynomial, quandles, the Khovanov homology, etc. It might be interesting to pose the following.

Problem 2.14. Extend various known knot invariants to those for profinite knots.

Profinite analogues of finite type knot invariants will be discussed in Remark 2.33 .

Below we remind one of the most elementary results for oriented knots.

Proposition 2.15. The space $\mathcal{K}$ of topological oriented knots carries a structure of a commutative associative monoid by the connected sum (knot sum).

Here the connected sum (knot sum) is a natural way to fuse two oriented knots, with an appropriate position of orientation, into one (an example is illustrated in Figure 2.15).

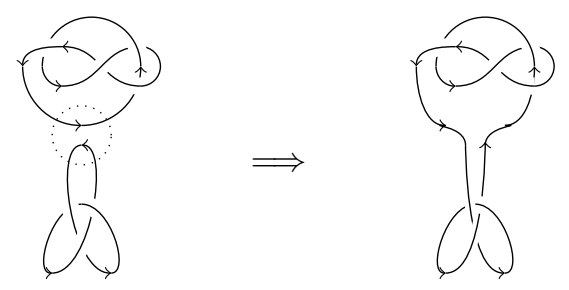

Figure 2.15. Connected sum (knot sum)

It can be done any points. It is actually well-defined. It yields a commutative monoid structure on $\mathcal{K}$. For more, consult the standard text book of knot theory.

The notion of connected sum can be extended into profinite knots.

Theorem 2.16. For any two profinite knots $K_{1}=\alpha_{m} \cdots \alpha_{1}$ and $K_{2}=\beta_{n} \cdots \beta_{1}$ with $\left(\alpha_{m}, \alpha_{1}\right)=(\curvearrowleft, \cup)$ and $\left(\beta_{n}, \beta_{1}\right)=(\curvearrowleft, \cup)$, their connected sum means the profinite tangle defined by

$$
K_{1} \sharp K_{2}:=\alpha_{m} \cdots \alpha_{2} \cdot \beta_{n-1} \cdots \beta_{1} .
$$

Then

(1). the above connected sum induces a well-defined product

$$
\sharp: \widehat{\mathcal{K}} \times \widehat{\mathcal{K}} \rightarrow \widehat{\mathcal{K}} .
$$

(2). By the product $\sharp$, the set $\widehat{\mathcal{K}}$ forms a topological (that is, the map $\sharp$ is continuous with respect to the topology given above) commutative associative monoid, whose unit is given by the oriented circle $\bigcirc:=\curvearrowleft \cdot \backsim$. 
(3). The profinite realization map $h: \mathcal{K} \rightarrow \widehat{\mathcal{K}}$ forms a monoid homomorphism whose image is dense in $\widehat{\mathcal{K}}$.

Proof. (1). Since each isotopy class of profinite knot contains by (T6) a profinite knot $K$ of the above type; a profinite knot starting with $\checkmark$ and ending at $\curvearrowleft$, we can show that the connected sum extends to $\widehat{\mathcal{K}}$ once we have the well-definedness.

Firstly we prove that $K_{1} \sharp K_{2}$ is isotopic to $K_{1}^{\prime} \sharp K_{2}$ if $K_{1}^{\prime}$ is isotopic to $K_{1}$, i.e., $K_{1}^{\prime}$ is obtained by a finite number of our moves (T1)-(T6) from $K_{1}$. We may assume that $K_{1}^{\prime}$ is obtained from $K_{1}$ by a single operation of one of the 6 moves. In the case where this move effects only on $\alpha_{i}$ 's for $i>1$, it is easy to see our claim. If the moves effects on $\alpha_{1}$, it must be (T3) or (T6). Consider the latter case (T6). It suffices to show that $K_{1} \sharp K_{2}$ is isotopic to $K_{3}=\alpha_{m} \cdots \alpha_{2} \cdot \sigma^{2 c} \cdot \beta_{n-1} \cdots \beta_{1}$ for $c \in \widehat{\mathbb{Z}}$. The proof is depicted in Figure 2.16 Here $S_{1}=\alpha_{m-1} \cdots \alpha_{2}$ and $S_{2}=\beta_{n} \cdots \beta_{2}$. We note that the first and the fourth equalities follow from (T3) and (T5). We use (T4) in the second equality for $\sigma^{2 c}$ and the dashed box. We derived the third equality from (T6).

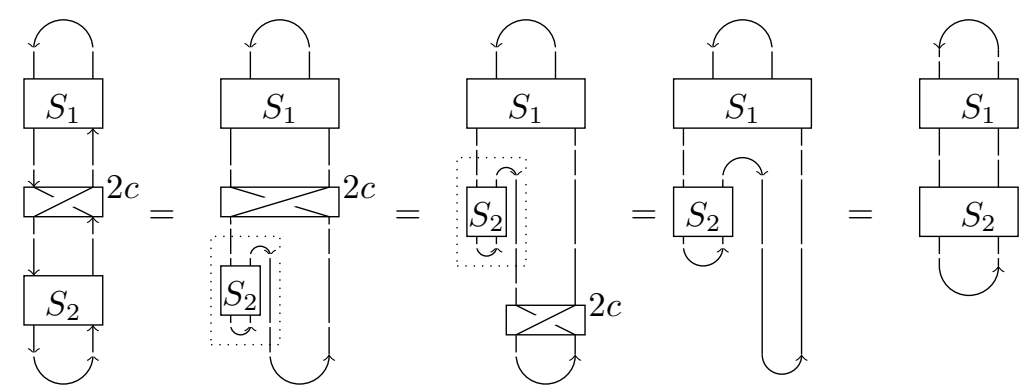

FiguRE 2.16. $K_{3}=K_{1} \sharp K_{2}$

Next consider the former case (T3). Since $K_{1}$ is a profinite knot, $m_{1}$ and $m_{2}$ are both 0 . By the above argument in case (T6), we may assume that both $T_{1}$ and $T_{2}$ in Figure 2.11 should start from $\smile$ (i.e. $\alpha_{1}=\beta_{1}=\smile$ ). Define $T$ as in Figure 2.17. A successive application of commutativity of profinite braids with $T$ shown in (T4) and that of creations and annihilations with $T$ shown in Lemma 2.18 lead the isotopy equivalence shown in Figure 2.18. Here $T_{1}^{\prime}$ and $T_{2}^{\prime}$ stand for emissions of the lowest $\checkmark$ from $T_{1}$ and $T_{2}$. We note that emission of $T$ in the figure represents $K_{1}$ and $K_{1}^{\prime}$.

Secondly we must prove that $K_{1} \sharp K_{2}$ is isotopic to $K_{1} \sharp K_{2}^{\prime}$ if $K_{2}^{\prime}$ is isotopic to $K_{2}$. It can be proved in a completely same way to the above arguments. Thus our proof is finally completed.

(2). Associativity, i.e. $\left(K_{1} \sharp K_{2}\right) \sharp K_{3}=K_{1} \sharp\left(K_{2} \sharp K_{3}\right)$, is easy to see. A proof of commutativity is illustrated in Figure 2.19. We note that we use (T3) and (T5) in the first, the third and the fifth equalities and we apply (T4) and Lemma 2.18 for the dashed boxes in the second and the fourth equalities.

To show that $\sharp$ is continuous, we define by $\widehat{\mathcal{T}}^{\text {seq }}$ the set of finite consistent sequences of profinite fundamental tangles and by $\widehat{\mathcal{K}}^{\text {seq }}$ the set of finite consistent sequences of profinite fundamental tangles $\gamma_{n} \cdots \gamma_{2} \cdot \gamma_{1}$ with a single connected component and with $\left(\gamma_{n}, \gamma_{1}\right)=(\curvearrowleft, \cup)$. We note that the quotient set of $\widehat{\mathcal{T}}^{\text {seq }}$ by 


$$
\frac{\uparrow}{T}=\overbrace{\uparrow}^{\uparrow \uparrow}
$$

Figure 2.17. definition of $T$

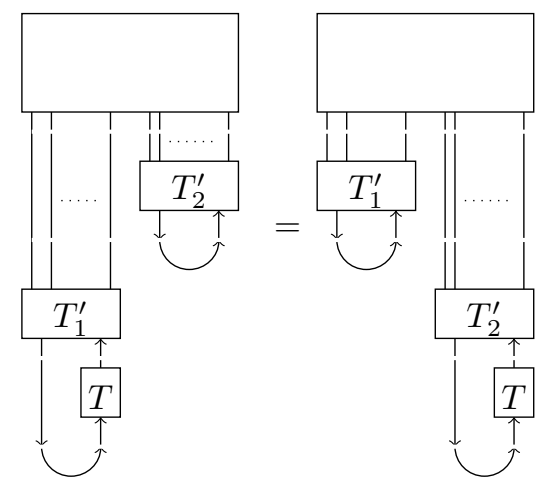

FiguRE 2.18. $K_{1} \sharp K_{2}=K_{1}^{\prime} \sharp K_{2}$

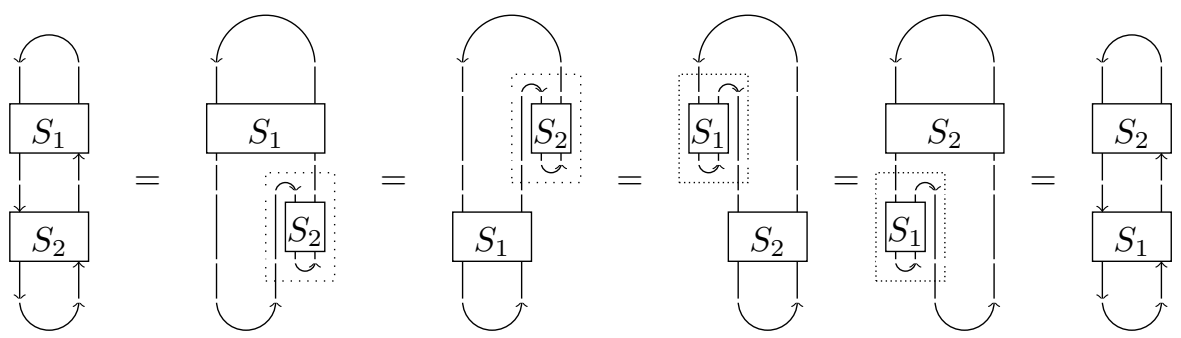

FiguRE 2.19. $K_{1} \sharp K_{2}=K_{2} \sharp K_{1}$

the equivalence of finite sequences of the moves (T1)-(T6) is equal to the set $\widehat{\mathcal{T}}$ of profinite tangles. We also note that $\widehat{\mathcal{K}}^{\text {seq }}$ is projected onto its subset $\widehat{\mathcal{K}}$ of profinite knots. The set $\widehat{\mathcal{T}}^{\text {seq }}$ carries a structure of a topological space by the profinite topologies on $\widehat{B}_{n}$ (Note 2.10). It induces a subspace topology on $\widehat{\mathcal{K}}^{\text {seq }}$. The map $\widehat{\mathcal{T}}^{\text {seq }} \rightarrow \widehat{\mathcal{T}}$ is continuous, hence so the projection $\widehat{\mathcal{K}}^{\text {seq }} \rightarrow \widehat{\mathcal{K}}$ is. By the topology it is easy to see that the map

$$
\sharp: \widehat{\mathcal{K}}^{\text {seq }} \times \widehat{\mathcal{K}}^{\text {seq }} \rightarrow \widehat{\mathcal{K}}^{\text {seq }}
$$

caused by (2.1) is continuous. Because the following diagram is commutative

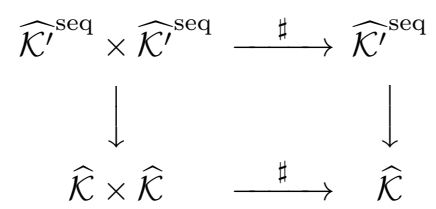

and the projection $\widehat{\mathcal{K}}^{\text {seq }} \rightarrow \widehat{\mathcal{K}}$ is continuous, the lower map is also continuous.

(3). The first statement is obvious. Let $\mathcal{K}^{\text {seq }}$ be the subset of $\widehat{\mathcal{K}}^{\text {seq }}$ which consists of consistent finite sequences of 'topological fundamental tangles', that is, sequences of elements in $A, C$ and $B$ with $b_{n} \in B_{n} \subset \widehat{B}_{n}$. There are a natural inclusion $\mathcal{K}^{\prime \text { seq }} \rightarrow \widehat{\mathcal{K}}^{\text {seq }}$ and a surjection $\mathcal{K}^{\text {seq }} \rightarrow \mathcal{K}$. Since the map $B_{n} \rightarrow \widehat{B}_{n}$ is with dense image, so the inclusion $\mathcal{K}^{\prime \text { seq }} \rightarrow \widehat{\mathcal{K}}^{\text {seq }}$ is. Therefore the map $\mathcal{K} \rightarrow \widehat{\mathcal{K}}$ is 
HIDEKAZU FURUSHO

also with dense image. It is because the following diagram is commutative

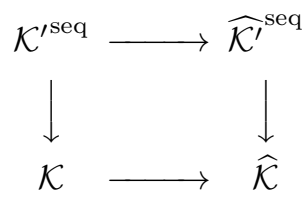

and the projection $\widehat{\mathcal{K}}^{\text {seq }} \rightarrow \widehat{\mathcal{K}}$ is continuous.

The following two lemmas are required to prove Theorem 2.16 (1).

Lemma 2.17. For a profinite tangle $T$ with $s(T)=t(T)=\uparrow$, define its transpose $L$ (occasionally also denoted by ${ }^{t} T$ ) by

$$
L:=a_{0,1}^{\curvearrowleft \downarrow} \cdot\left(e_{1}^{\downarrow} \otimes T \otimes e_{1}^{\downarrow}\right) \cdot c_{1,0}^{\downarrow \smile} .
$$

Then we have

$$
L=a_{1,0}^{\downarrow \curvearrowright} \cdot\left(e_{1}^{\downarrow} \otimes T \otimes e_{1}^{\downarrow}\right) \cdot c_{0,1}^{\curlyvee} .
$$

Similar claim holds for a profinite tangle $T$ with $s(T)=t(T)=\downarrow$ by reversing all arrows.

Figure 2.20 describes our lemma.

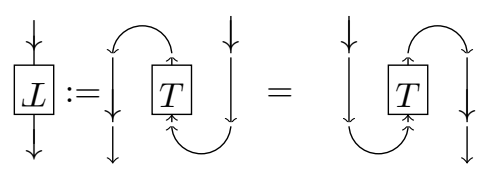

Figure 2.20. transpose of $T$

Proof. A proof is depicted in Figure 2.21. We note that we apply (T6) in the first equality and (T2) and (T4) in the second equality.

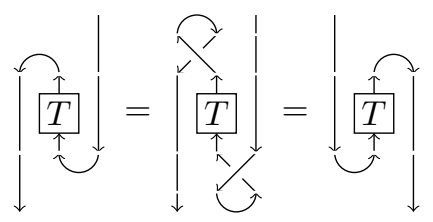

FigurE 2.21. proof of Lemma 2.17

By (T5) and the above lemma, we see that ${ }^{t t} T$ is isotopic to $T$.

Lemma 2.18. For a profinite tangle $T$ with $s(T)=t(T)=\uparrow$, the equalities in Figure 2.22 hold. The same claim also holds for a profinite tangle $T$ with $s(T)=$ $t(T)=\downarrow$ by reversing all arrows.

Proof. It can be checked by direct computation using Lemma 2.17

In knot theory, the so-called Alexander-Markov's theorem is fundamental on constructions of knot invariants. The theorem is to translate knots and links into purely algebraic objects: 

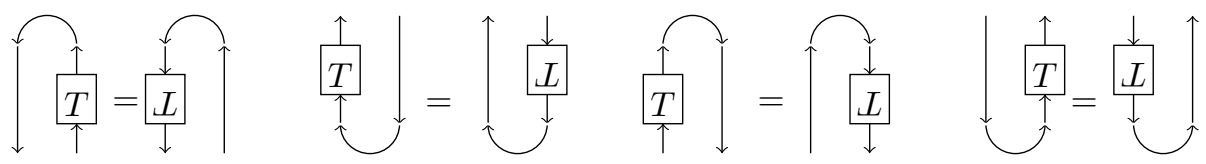

Figure 2.22. Creation and annihilation commute with $T$.

Theorem 2.19 (Alexander-Markov's theorem). There is a one-to-one correspondence

$$
\mathcal{L} \longleftrightarrow \sqcup_{n} B_{n} / \sim_{M}
$$

between the set $\mathcal{L}$ of isotopy classes of oriented links and the (disjoint) union $\sqcup_{n} B_{n}$ of braids groups modulo the equivalence $\sim_{M}$ given by the following Markov moves

(M1). $b_{1} \cdot b_{2} \sim_{M} b_{2} \cdot b_{1} \quad\left(b_{1}, b_{2} \in B_{n}\right), \quad\left(\right.$ M2). $b \in B_{n} \sim_{M} b \sigma_{n}^{ \pm 1} \in B_{n+1} \quad\left(b \in B_{n}\right)$

For more on the theorem, consult [CDM, O1] for example. The question below is to ask a validity of profinite analogue of Alexander-Markov's theorem.

Problem 2.20. Is there a 'profinite analogue' of the Alexander-Markov's theorem which holds for the set $\widehat{\mathcal{L}}$ of isotopy classes of profinite links ?

There are several proofs of Alexander-Markov's theorem for topological links ([Bi, $\operatorname{Tr}, \mathrm{V}, \mathrm{Y}]$ etc). But they look heavily based on a certain finiteness property, which we (at least the author) may not expect the validity for profinite links.

2.2. Pro- $l$ knots. Pro- $l$ tangle diagrams, pro- $l$ knot diagrams and isotopy among them which are pro- $l$ analogues of our corresponding notions given in the previous subsection, are introduced in Definition 2.25. A natural map from profinite tangles (resp. profinite knots) to pro- $l$ tangles (resp. pro- $l$ knots) is constructed in Proposition 2.26. Proalgebraic tangles and proalgebraic knots are recalled in Definition 2.30. A natural map from pro- $l$ tangles (resp. pro- $l$ knots) to proalgebraic tangles (resp. proalgebraic knots) is given in Proposition 2.31 It is explained that the Kontsevich invariant factors through these natural maps in Remark 2.34. Our discussion in this subsection will serve for a proof of non-triviality of the Galois action constructed in 2.3 .

Let $l$ be a prime. We may include $l=2$.

Notation 2.21. A topological group $G$ is called a pro-l group if it is a projective $\operatorname{limit} \lim G_{i}$ of a projective system of finite $l$-groups $\left\{G_{i}\right\}_{i \in I}$. For a discrete group $\Gamma$, its pro-l completion $\widehat{\Gamma}^{l}$ is the pro-l group defined by the projective limit

$$
\widehat{\Gamma}^{l}=\lim _{\longleftarrow} \Gamma / N
$$

where $N$ runs over all normal subgroups of $\Gamma$ with finite indices of power of $l$.

For more on pro- $l$ groups, consult $[\mathrm{RZ}]$ for example. We note there is a natural homomorphism $\Gamma \rightarrow \widehat{\Gamma}^{l}$.

Notation 2.22. Let $n \geqslant 2$. Let $\widehat{P}_{n} \rtimes B_{n}$ be the semi-direct product of $\widehat{P}_{n}$ and $B_{n}$ with respect to the $B_{n}$-action on $\widehat{P}_{n}$ given by $p \mapsto b p b^{-1}\left(p \in \widehat{P}_{n}\right.$ and $\left.b \in B_{n}\right)$. consider the inclusion $P_{n} \hookrightarrow \widehat{P}_{n} \rtimes B_{n}$ sending $p \mapsto\left(p, p^{-1}\right)$. Then it is easy to see the homomorphism sending $(p, b) \mapsto p b$ yields an isomorphism:

$$
\left(\widehat{P}_{n} \rtimes B_{n}\right) / P_{n} \simeq \widehat{B}_{n} .
$$


Definition 2.23. (1). The pro-l pure braid group $\widehat{P}_{n}^{l}$ is the pro-l completion of $P_{n}$.

(2). The pro-(l) braid group $\widehat{B}_{n}^{(l)}$ is defined to be the induced quotient

$$
\widehat{B}_{n}^{(l)}:=\left(\widehat{P}_{n}^{l} \rtimes B_{n}\right) / P_{n} .
$$

We encode a topological group structure on $\widehat{B}_{n}^{(l)}$ by the pro-l topology on $\widehat{P}_{n}^{l}$ and the discrete topology on $B_{n}$. We note that this $\widehat{B}_{n}^{(l)}$ appears also in [LS.

Remark 2.24. (1). Our $\widehat{B}_{n}^{(l)}$ is different from the pro-l completion $\widehat{B}_{n}^{l}$ of $B_{n}$.

(2). There is an exact sequence:

$$
1 \rightarrow \widehat{P}_{n}^{l} \rightarrow \widehat{B}_{n}^{(l)} \rightarrow \mathfrak{S}_{n} \rightarrow 1
$$

(3). There are natural group homomorphisms:

$$
B_{n} \rightarrow \widehat{B}_{n} \rightarrow \widehat{B}_{n}^{(l)} .
$$

The map (2.2) is induced from $P_{n} \rightarrow \widehat{P}_{n} \rightarrow \widehat{P}_{n}^{l}$.

Definition 2.25. (1). A pro-l tangle diagram means a consistent finite sequence of fundamental pro-l tangle diagrams, which are elements in $A, C$ (in Definition 2.2) or

$$
\widehat{B}^{l}:=\left\{b_{n}^{\epsilon} \mid b_{n}^{\epsilon}=\left(b_{n}, \epsilon=\left(\epsilon_{i}\right)_{i=1}^{n}\right) \in \widehat{B}_{n}^{(l)} \times\{\uparrow, \downarrow\}^{n}, n=1,2,3,4, \ldots\right\} .
$$

A pro-l knot diagram means a pro-l tangle diagram without endpoints (their sources and targets are both empty) and with a single connected component.

(2). Two pro-l tangle diagrams $T_{1}$ and $T_{2}$ are said to be isotopic if they are related by a finite number of the moves replacing profinite tangles and profinite braids by pro-l tangles and elements in $\widehat{B}^{l}$ in (T1)-(T6) and $c \in \widehat{\mathbb{Z}}$ by $c \in \mathbb{Z}_{l}$ in (T6). 9

(3). A pro-l tangle (resp. pro-l) means an isotopic class of pro-l tangle (resp. knot) diagram. We denote the set of pro-l tangles by $\widehat{\mathcal{T}}^{l}$ and the set of pro-l knots by $\widehat{\mathcal{K}}^{l}$.

Both $\widehat{\mathcal{T}}^{l}$ and $\widehat{\mathcal{K}}^{l}$ carry a structure of topological space by the method in Note 2.10 .

Proposition 2.26. (1). The set $\widehat{\mathcal{K}}^{l}$ forms a topological monoid with respect to the connected sum.

(2). There are continuous maps:

$$
\begin{aligned}
\widehat{\mathcal{T}} & \rightarrow \widehat{\mathcal{T}}^{l}, \\
\widehat{\mathcal{K}} & \rightarrow \widehat{\mathcal{K}}^{l} .
\end{aligned}
$$

(3). The map (2.4) is monoid homomorphisms. The image of its composition with $h$ in Theorem 2.11. (2)

$$
h_{l}: \mathcal{K} \stackrel{h}{\rightarrow} \widehat{\mathcal{K}} \rightarrow \widehat{\mathcal{K}}^{l} .
$$

is with dense image in $\widehat{\mathcal{K}}^{l}$.

\footnotetext{
${ }^{9}$ We note that, for $\sigma_{i} \in \widehat{B}_{N}^{(l)}$ and $c \in \mathbb{Z}_{l}$, the power $\sigma_{i}^{2 c+1}$ makes sense in $\widehat{B}_{N}^{(l)}$ because, by $\sigma_{i}^{2} \in \widehat{P}_{N}^{l}$, we have $\sigma_{i}^{2 c} \in \widehat{P}_{N}^{l}$.
} 
Proof. (1). It is obtained by the same arguments to the proof of Theorem 2.16.

(2). The map (2.3) is induced from the second map in (2.2), whose continuity implies ours. It preserves each connected component, which yields the map (2.4).

(3). To see that they form homomorphisms are immediate. The density can be proved by the same arguments to the proof of Theorem 2.16 .

Definition 2.27. Let $R$ be a commutative ring.

(1). Let $I$ be the two-sided ideal of the group algebra $R\left[B_{n}\right]$ of $B_{n}$ generated by $\sigma_{i}-\sigma_{i}^{-1}(1 \leqslant i \leqslant n-1)$. The topological $R$-algebra $\widehat{R\left[B_{n}\right]}$ of proalgebraic braids means its completion with respect to the $I$-adic filtration, i.e.

$$
\widehat{R\left[B_{n}\right]}:=\lim _{\overleftarrow{N}} R\left[B_{n}\right] / I^{N}
$$

(2). Put $I_{0}:=I \cap R\left[P_{n}\right]$. Then $I_{0}$ is the augmentation ideal of $R\left[P_{n}\right]$ (cf. $[\mathrm{KT}$ ). The topological $R$-algebra $\widehat{R\left[P_{n}\right]}$ of proalgebraic pure braids means its completion

$$
\widehat{R\left[P_{n}\right]}:=\lim _{\overleftarrow{N}} R\left[P_{n}\right] / I_{0}^{N}
$$

It is a subalgebra of $\widehat{R\left[B_{n}\right]}$.

It is direct to see that both algebras naturally equip structures of co-commutative Hopf algebras.

Remark 2.28. (i). There is a short exact sequence

$$
0 \rightarrow \widehat{R\left[P_{n}\right]} \rightarrow \widehat{R\left[B_{n}\right]} \rightarrow R\left[\mathfrak{S}_{n}\right] \rightarrow 0 .
$$

(ii). We remark that (the group-like part of) $\widehat{R\left[P_{n}\right]}$ is the unipotent (Malcev) completion of $P_{n}$ and (the group-like part of) $\widehat{R\left[B_{n}\right]}$ is Hain's $\underline{\mathrm{H}}$ ] relative completion of $B_{n}$ with respect to the natural projection $B_{n} \rightarrow \mathfrak{S}_{n}$.

(iii). The natural morphisms $P_{n} \rightarrow R\left[P_{n}\right]$ and $B_{n} \rightarrow R\left[B_{n}\right]$ yield injections

$$
P_{n} \hookrightarrow \widehat{R\left[P_{n}\right]} \quad \text { and } \quad B_{n} \hookrightarrow \widehat{R\left[B_{n}\right]}
$$

(cf. CDM $\S 12$ ).

Since $\widehat{P}_{n}^{l}$ is a pro-l group and $I_{0}$ is the augmentation ideal, we have a natural continuous homomorphism $\widehat{P}_{n}^{l} \rightarrow \widehat{\mathbb{Q}_{l}\left[P_{n}\right]}$ (cf. [CDM] for example).

Proposition 2.29. There is a natural continuous group homomorphism

$$
\widehat{B}_{n}^{(l)} \rightarrow \widehat{\mathbb{Q}_{l}\left[B_{n}\right]} .
$$

Proof. It can be directly checked that the map induced from the above $\widehat{P}_{n}^{l} \rightarrow \widehat{\mathbb{Q}_{l}\left[P_{n}\right]}$ $\left(\subset \widehat{\mathbb{Q}_{l}\left[B_{n}\right]}\right)$ and the natural map $B_{n} \hookrightarrow \mathbb{Q}_{l}\left[B_{n}\right] \rightarrow \widehat{\mathbb{Q}_{l}\left[B_{n}\right]}$ holds the property.

Next we discuss the corresponding notions in tangles and knots settings. The following notions go back to the idea of Vassiliev.

Definition 2.30 ( $[\mathrm{KT}]$ ). Let $R$ be a commutative ring. Let $\mathcal{T}_{\epsilon, \epsilon^{\prime}}$ be the full set of isotopy classes of oriented tangles with type $\left(\epsilon, \epsilon^{\prime}\right)$. 
(1). Let $R\left[\mathcal{T}_{\epsilon, \epsilon^{\prime}}\right]$ be the free $R$-module of finite formal sums of elements of $\mathcal{T}_{\epsilon, \epsilon^{\prime}}$. A singular oriented tangle 10 determines an element of $R\left[\mathcal{T}_{\epsilon, \epsilon^{\prime}}\right]$ by the desingularization of each double point by the following relation

$$
X=Y-X
$$

Let $\mathcal{T}_{n}(n \geqslant 0)$ be the $R$-submodule of $R\left[\mathcal{T}_{\epsilon, \epsilon^{\prime}}\right]$ generated by all singular oriented tangles with type $\left(\epsilon, \epsilon^{\prime}\right)$ and with $n$ double points. The descending filtration $\left\{\mathcal{T}_{n}\right\}_{n} \geqslant 0$ is called the singular filtration. The topological $R$-module $\widehat{R\left[\mathcal{T}_{\epsilon, \epsilon^{\prime}}\right]}$ of proalgebraic tangles means its completion with respect to the singular filtration:

$$
\widehat{R\left[\mathcal{T}_{\epsilon, \epsilon^{\prime}}\right]}:=\lim _{\overleftarrow{N}} R\left[\mathcal{T}_{\epsilon, \epsilon^{\prime}}\right] / \mathcal{T}_{N}
$$

(2). Let $R[\mathcal{K}]$ be the $R$-submodule of $R\left[\mathcal{T}_{\emptyset, \emptyset]}\right.$ generated by elements of $\mathcal{K}$. By Proposition 2.15, it forms a commutative $R$-algebra. Put $\mathcal{K}_{n}:=\mathcal{T}_{n} \cap R[\mathcal{K}](n \geqslant 0)$. Then $\mathcal{K}_{n}$ forms an ideal of $R[\mathcal{K}]$ and the descending filtration $\left\{\mathcal{K}_{n}\right\}_{n \geqslant 0}$ is called the singular knot filtration (cf. loc.cit.). The topological commutative $R$-algebra $\widehat{R[\mathcal{K}]}$ of proalgebraic knots means its completion with respect to the singular knot filtration:

$$
\widehat{R[\mathcal{K}]}:=\lim _{N} R[\mathcal{K}] / \mathcal{K}_{N}
$$

Actually it equips a structure of co-commutative and commutative Hopf algebra.

The maps below are tangle and knot analogues of the map (2.7).

Proposition 2.31. (1). There are continuous maps:

$$
\begin{aligned}
& \widehat{\mathcal{T}}^{l} \rightarrow \widehat{\mathbb{Q}_{l}[\mathcal{T}]}, \\
& \widehat{\mathcal{K}}^{l} \rightarrow \widehat{\mathbb{Q}_{l}[\mathcal{K}]} .
\end{aligned}
$$

(2). The map (2.9) is a continuous monoid homomorphism and its image lies

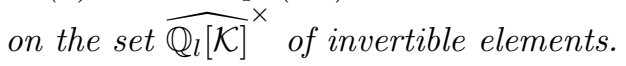

Proof. (1). Since an element $b_{n}^{\epsilon} \in B_{n} \times\{\uparrow, \downarrow\}^{n}(n \geqslant 1)$, a braid $b_{n} \in B_{n}$ with an orientation $\epsilon \in\{\uparrow, \downarrow\}^{n}$ (namely its source), is naturally regarded as a special type of an oriented tangle, each orientation $\epsilon$ yields a natural inclusion

$$
\mathbb{Q}_{l}\left[B_{n}\right] \hookrightarrow \mathbb{Q}_{l}[\mathcal{T}] .
$$

On the embedding, we have $\mathcal{T}_{m} \cap \mathbb{Q}_{l}\left[B_{n}\right]=I^{m}$ for $m \geqslant 0$. Therefore the above map and the map (2.7) induce

$$
\widehat{B}_{n}^{(l)} \rightarrow \mathbb{Q}_{l}[\mathcal{T}] / \mathcal{T}_{m}
$$

Hence it determines the map of sets

$$
\widehat{B}^{l} \rightarrow \mathbb{Q}_{l}[\mathcal{T}] / \mathcal{T}_{m}
$$

We also have the natural maps of sets

$$
A \rightarrow \mathbb{Q}_{l}[\mathcal{T}] / \mathcal{T}_{m} \text { and } C \rightarrow \mathbb{Q}_{l}[\mathcal{T}] / \mathcal{T}_{m}
$$

As is described in the proof of Theorem 2.11, the set $\mathcal{T}$ of (topological) oriented tangles is described by the set of consistent finite sequences of elements of $A, B$ and

10 It is an 'oriented tangle' which is allowed to have a finite number of transversal double points (see $[\mathrm{KT}]$ for precise). 
$C$ modulo the (discrete) Turaev moves. By $\mathbb{Q}_{l}$-linearly extending the description, we obtain the same description of $\mathbb{Q}_{l}[\mathcal{T}]$. Since our three maps (2.10) and (2.11) are consistent with the moves, we obtain

$$
\widehat{\mathcal{T}}^{l} \rightarrow \mathbb{Q}_{l}[\mathcal{T}] / \mathcal{T}_{m}
$$

(Again we note that, for $\sigma_{i} \in B_{N}$ and $c \in \mathbb{Z}_{l}$, the power $\sigma_{i}^{c}$ makes sense in $\mathbb{Q}_{l}\left[B_{N}\right] / I^{m}$ by the formula

$$
\sigma_{i}^{c}:=\exp \left\{\frac{c}{2} \log \sigma_{i}^{2}\right\}
$$

when $l \neq 2$ or when $l=2$ and $c \in 2 \mathbb{Z}_{2}$, and

$$
\sigma_{i}^{c}:=\sigma_{i} \cdot \exp \left\{\frac{c-1}{2} \log \sigma_{i}^{2}\right\}
$$

when $l=2$ and $c \notin 2 \mathbb{Z}_{2}$. Here exp and $\log$ are defined by the usual Taylor expansions. The RHS is well-defined by $\sigma_{i}^{2}-1 \in I$.)

It yields the map (2.8) which is continuous. Since this map preserves each connected component, the map (2.9) is also obtained.

(2). It is immediate to see that it forms a continuous homomorphism.

Each oriented knot, an element of $\mathcal{K}$, is congruent to the unit, the trivial knot $\circlearrowleft \in \mathbb{Q}_{l}[\mathcal{K}]$, modulo $\mathcal{K}_{1}$, because any knot can be untied by a finite times of changing crossings (consult for unknotting number, say, in CDM $)$. Therefore the image of $h_{l}(\mathcal{K})\left(\subset \widehat{\mathcal{K}}^{l}\right)$ is contained in the subspace $\bigcirc+\mathcal{K}_{1} \cdot \widehat{\mathbb{Q}_{l}[\mathcal{K}]}$. Hence the image of $\widehat{\mathcal{K}}^{l}$ should lie on the subspace. It is because the subspace is open in $\widehat{\mathbb{Q}_{l}[\mathcal{K}]}$, our map (2.9) is continuous as shown above and $h_{l}(\mathcal{K})$ is dense in $\widehat{\mathcal{K}}_{l}$ by Proposition 2.26. (3). All elements of the subspace are invertible because it is known that the quotient $\widehat{\mathbb{Q}_{l}[\mathcal{K}]} / \mathcal{K}_{1}$ is 1 -dimensional and generated by $\bigcirc$. Thus the claim is obtained.

The author is not aware if our above two maps are injective or not.

Proposition 2.32. (1). For each prime l, there are continuous maps:

$$
\begin{aligned}
& \widehat{\mathcal{T}} \rightarrow \widehat{\mathbb{Q}_{l}[\mathcal{T}]}, \\
& \widehat{\mathcal{K}} \rightarrow \widehat{\mathbb{Q}_{l}[\mathcal{K}]} .
\end{aligned}
$$

(2). The map (2.13) is a continuous monoid homomorphism and its image lies on the set ${\widehat{\mathbb{Q}_{l}[\mathcal{K}]}}^{\times}$of invertible elements.

(3). The image of the composition of (2.13) with $h$ in Theorem 2.11.(2)

$$
\mathcal{K} \stackrel{h}{\rightarrow} \widehat{\mathcal{K}} \rightarrow \widehat{\mathbb{Q}_{l}[\mathcal{K}]} .
$$

lies on the rational invertible part $\widehat{\mathbb{Q}[\mathcal{K}]}^{\times}\left(\subset \widehat{\mathbb{Q}_{l}[\mathcal{K}]}\right)$. Furthermore the resulting morphism

$$
i: \mathcal{K} \rightarrow \widehat{\mathbb{Q}[\mathcal{K}]}
$$

is independent of a prime $l$.

Proof. (1) and (2) follow from Proposition 2.26 and 2.31, Our claim (3) follows from that the map (2.14) is induced from the natural inclusion $\mathcal{K} \hookrightarrow R[\mathcal{K}]$ with $R=\mathbb{Q}$. 
Relating to Problem 2.14, the following is obtained as an application of Proposition 2.32 .

Remark 2.33. (1). Finite type knot invariants (resp. and their projective limits) valued on $\mathbb{Q}$ are knot invariants which factor through $\mathcal{K} \rightarrow \widehat{\mathbb{Q}[\mathcal{K}]} \rightarrow \mathbb{Q}[\mathcal{K}] / \mathcal{K}_{N}$ for some $N$ (resp. $\mathcal{K} \rightarrow \widehat{\mathbb{Z}[\mathcal{K}]}$ ). By using the map (2.14), we can extend all finite type knot invariants and their projective limits, such as each coefficient of the Jones polynomial substituting $e^{h}$ (cf. $[\mathrm{CDM}]$ ), into profinite knot invariants valued on $\mathbb{Q}_{l}$ for each prime $l$. It is easy to see that the same holds for tangle invariants.

(2). The linking number is an invariant of two components links which values on $\mathbb{Z}$ and which is known to be of finite type ([CDM], etc). Thus it can be extended to an invariant of two components profinite links which values on $\mathbb{Q}_{l}$, actually on $\mathbb{Z}_{l}$, for each prime $l$. The Milnor $\bar{\mu}$-invariant [Mi1, Mi2] is an invariant of links which is known as a higher generalization of the linking number. It is defined on $\mathbb{Z}$ but it has a certain indeterminacy. The author is not sure if it can be also extended to an invariant of profinite links, but he expects that the works $\mathrm{HL}, \mathrm{Ba} 2$, Lin concerning its associated invariant of string links would help to detect that.

As a complementation of Remark 2.13, we have

Remark 2.34. The Kontsevich invariant $Z: \mathcal{K} \rightarrow \widehat{C D}$ is a knot invariant which is a composition of $i$ with the isomorphism $\widehat{\mathbb{Q}[\mathcal{K}]} \simeq \widehat{C D}$ constructed in $[\mathrm{K}$. Here $\widehat{C D}$ stands for the $\mathbb{Q}$-vector space (completed by degree) of chord diagrams modulo 4T- and FI-relations (consult also CDM, O1). The invariant is conjectured to be perfect, i.e., the map $Z$ is conjectured to be injective (cf. O2] Conjecture 3.2). The conjecture is equivalent to saying that the above map $i$ in (2.15) is injective. Hence it naturally leads us to conjecture that $h: \mathcal{K} \rightarrow \widehat{\mathcal{K}}$ is injective (Conjecture 2.12) because the non-injectivity of $h$ imply the non-injectivity of $i$, that is, the non-perfectness of the Kontsevich invariant, by the above proposition. We may also say that Conjecture 2.12 is an assertion on a knot analogue of the injectivity of the maps (2.6).

2.3. Action of the absolute Galois group. The group Frac $\widehat{\mathcal{K}}$ of profinite knots is introduced as the group of fraction of the topological monoid $\widehat{\mathcal{K}}$ in Definition 2.35 and its basic property is shown in Theorem 2.36. A continuous action of the profinite Grothendieck-Teichmüler group $\widehat{G T}$ (cf. Definition [1.6) on Frac $\widehat{\mathcal{K}}$ is established in Definition 2.39. Theorem 2.41, As a result of our construction, an action of the absolute Galois group $G_{\mathbb{Q}}$ of the rational number field on Frack $\widehat{\mathcal{K}}$ is obtained (Theorem 2.45). We post several projects and problems on this Galois representation in the end.

Definition 2.35. The group of profinite knots Frac $\widehat{\mathcal{K}}$ is defined to be the group of fraction of the monoid $\widehat{\mathcal{K}}$, i.e., the quotient space of $\widehat{\mathcal{K}}^{2}$ by the equivalent relations $(r, s) \approx\left(r^{\prime}, s^{\prime}\right)$ if $r \sharp s^{\prime} \sharp t \sim r^{\prime} \sharp s \sharp t$ for some profinite knot $t$, i.e. $r \sharp s^{\prime} \sharp t=r^{\prime} \sharp s \sharp t$ holds in $\widehat{\mathcal{K}}$. Occasionally we denote the equivalent class $[(r, s)]$ by $\frac{r}{s}$.

For $\left[\left(r_{1}, s_{1}\right)\right]$ and $\left[\left(r_{2}, s_{2}\right)\right] \in$ Frac $\widehat{\mathcal{K}}$, define its product by

$$
\left[\left(r_{1}, s_{1}\right)\right] \sharp\left[\left(r_{2}, s_{2}\right)\right]:=\left[\left(r_{1} \sharp r_{2}, s_{1} \sharp s_{2}\right)\right] \in \operatorname{Frac} \widehat{\mathcal{K}}, \quad \text { i.e. } \quad \frac{r_{1}}{s_{1}} \sharp \frac{r_{2}}{s_{2}}=\frac{r_{1} \sharp r_{2}}{s_{1} \sharp s_{2}} \in \operatorname{Frac} \widehat{\mathcal{K}} .
$$

We encode Frac $\widehat{\mathcal{K}}$ with the quotient topology of $\widehat{\mathcal{K}}^{2}$. 
Theorem 2.36. (1). The product $\sharp$ is well-defined on Frac $\widehat{\mathcal{K}}$. The set Frac $\widehat{\mathcal{K}}$ forms a topological commutative group.

(2). It is a non-trivial group. Actually it is an infinite group.

Proof. (1). It is easy to see that $\sharp$ is well-defined and Frac $\widehat{\mathcal{K}}$ forms a commutative group with unit

by Theorem 2.16

$$
e=(\bigcirc, \bigcirc)
$$

Consider the commutative diagram

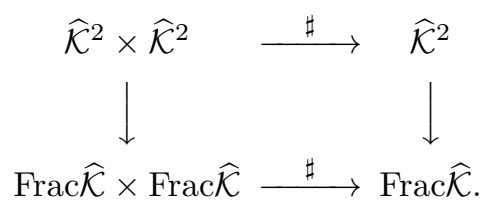

Since the upper map is continuous by Theorem 2.16 and the surjection $\widehat{\mathcal{K}}^{2} \rightarrow$ Frac $\widehat{\mathcal{K}}$ is continuous by definition, it follows that the map $\sharp$ is continuous.

Let $\tau: \widehat{\mathcal{K}}^{2} \rightarrow \widehat{\mathcal{K}}^{2}$ be the switch map sending $(r, s) \mapsto(s, r)$. It is easy to see that it is continuous and it induces the inverse map on Frac $\widehat{\mathcal{K}}$. Then by the commutative diagram

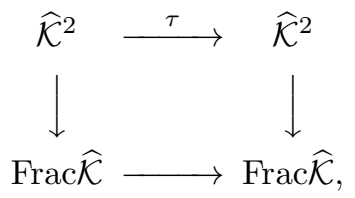

the inverse map is also continuous.

(2). By Proposition 2.26 and 2.31, there is a continuous monoid homomorphism

$$
\widehat{\mathcal{K}} \rightarrow \widehat{\mathcal{K}}^{l} \rightarrow \widehat{\mathbb{Q}_{l}[\mathcal{K}]}
$$

for a prime $l$. By Proposition 2.31, the image lies on ${\widehat{\mathbb{Q}_{l}[\mathcal{K}]}}^{\times}$. Whence it induces a continuous group homomorphism

$$
\operatorname{Frac} \widehat{\mathcal{K}} \rightarrow{\widehat{\mathbb{Q}}\left[{ }_{l}[\mathcal{K}]\right.}^{\times} .
$$

Thus it is enough to show that the image of the composition of the maps (2.16) and $h: \mathcal{K} \rightarrow \widehat{\mathcal{K}}$ is infinite set. The claim is obvious because this map is equal to $i$ in (2.15) and the Kontsevich invariant takes infinite number of values (cf. Remark 2.34)

A reason why we introduce Frac $\widehat{\mathcal{K}}$ is that we need to treat the inverse of $\Lambda_{f}$ in Figure 2.25 when we let $\widehat{G T}$ act on profinite knots (cf. Definition 2.39).

We note that the natural morphism

$$
h^{\prime}: \mathcal{K} \rightarrow \operatorname{Frac} \widehat{\mathcal{K}}
$$

sending $K \mapsto[(K, \bigcirc)]$ is a homomorphism as monoid. By abuse of notations, we occasionally denote the image $h^{\prime}(K)$ by the same symbol $K$. Related to Conjecture 2.12 ,

Problem 2.37. Is the map $h^{\prime}$ injective?

On a structure of Frac $\widehat{\mathcal{K}}$, we pose 
Problem 2.38. Is Frac $\widehat{\mathcal{K}}$ a profinite group?

By [RZ, to show that Frac $\widehat{\mathcal{K}}$ is a profinite group, we must show that it is compact, Hausdorff and totally-disconnected. The author is not aware of any one of their validities. It is worthy to note that the set $\widehat{\mathcal{T}}$ of isotopy classes of profinite tangles is not compact, hence not a profinite space. It is because the map $\left|\pi_{0}\right|: \widehat{\mathcal{T}} \rightarrow \mathbb{N}$ taking the number of connected components of each profinite tangles is continuous and surjective to the non-compact space $\mathbb{N}$.

Definition 2.39. Let $(r, s)$ be a pair of profinite knot diagrams with $r=\gamma_{1, m} \cdots \gamma_{1,2}$. $\gamma_{1,1}$ and $s=\gamma_{2, n} \cdots \gamma_{2,2} \cdot \gamma_{2,1}$ ( $\gamma_{i, j}$ : profinite fundamental tangle diagram). For $\sigma=(\lambda, f) \in \widehat{G T}$ (hence $\lambda \in \widehat{\mathbb{Z}}^{\times}, f \in \widehat{F}_{2}$ ), define its action by

$$
\sigma\left(\frac{r}{s}\right):=\frac{\sigma(r)}{\sigma(s)}:=\frac{\left\{\sigma\left(\gamma_{1, m}\right) \cdots \sigma\left(\gamma_{1,2}\right) \cdot \sigma\left(\gamma_{1,1}\right)\right\} \sharp\left(\Lambda_{f}\right)^{\sharp \alpha(s)}}{\left\{\sigma\left(\gamma_{2, n}\right) \cdots \sigma\left(\gamma_{2,2}\right) \cdot \sigma\left(\gamma_{2,1}\right)\right\} \sharp\left(\Lambda_{f}\right)^{\sharp \alpha(r)}} \in \text { Frac } \widehat{\mathcal{K}} .
$$

It is well-defined by Proposition 2.40 and Theorem 2.41 Here

$\sigma(r):=\frac{\left\{\sigma\left(\gamma_{1, m}\right) \cdots \sigma\left(\gamma_{1,2}\right) \cdot \sigma\left(\gamma_{1,1}\right)\right\}}{\left(\Lambda_{f}\right)^{\sharp \alpha(r)}}$ and $\sigma(s):=\frac{\left\{\sigma\left(\gamma_{2, n}\right) \cdots \sigma\left(\gamma_{2,2}\right) \cdot \sigma\left(\gamma_{2,1}\right)\right\}}{\left(\Lambda_{f}\right)^{\sharp \alpha(s)}} \in$ Frac $\widehat{\mathcal{K}}$ are defined as follows:

(1) When $\gamma_{i, j}=a_{k, l}^{\epsilon}$, we define

$$
\sigma\left(\gamma_{i, j}\right):=\gamma_{i, j} \cdot f_{1 \ldots k, k+1, k+2}^{s\left(\gamma_{i, j}\right)}
$$

Here $f_{1 \cdots k, k+1, k+2}^{s\left(\gamma_{i, j}\right)}=\operatorname{ev}_{1, \epsilon_{1}}\left(f^{\uparrow \epsilon_{2} \epsilon_{3}}\right) \otimes e_{l}^{\epsilon_{4}}$ with $s\left(\gamma_{i, j}\right)=\epsilon_{1} \epsilon_{2} \epsilon_{3} \epsilon_{4} \in\{\uparrow, \downarrow$ \}$^{k+l+2}\left(\epsilon_{1} \in\{\uparrow, \downarrow\}^{k}, \epsilon_{2}, \epsilon_{3} \in\{\uparrow, \downarrow\}, \epsilon_{4} \in\{\uparrow, \downarrow\}^{l}\right)$. It is also described by $f_{1 \cdots k, k+1, k+2}^{s\left(\gamma_{i, j}\right)}=\left(f_{1 \cdots k, k+1, k+2} \otimes e_{l}, s\left(\gamma_{i, j}\right)\right) \in \widehat{B}$ with

$$
f_{1 \cdots k, k+1, k+2} \otimes e_{l}=f\left(x_{1 \cdots k, k+1}, x_{k+1, k+2}\right) \in \widehat{B}_{k+l+2}
$$

where $x_{1 \ldots k, k+1}$ and $x_{k+1, k+2}$ are regarded as elements of $\widehat{B}_{k+l+2}$. We mean $f_{1 \cdots k, k+1, k+2} \otimes e_{l}$ by the trivial braid $e_{l+2} \in \widehat{B}_{l+2}$ when $k=0$. Figure 2.23 depicts the action. Here the thickened black band stands for the trivial braid $e_{k}$ with $k$-strings.

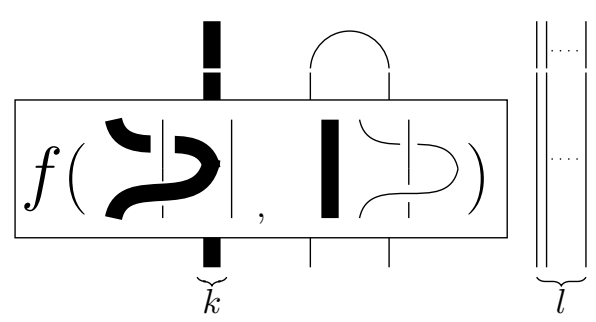

Figure 2.23. $\sigma\left(a_{k, l}^{\epsilon}\right)$

(2) When $\gamma_{i, j}=b_{n}^{\epsilon}=\left(b_{n}, \epsilon\right) \in \widehat{B}$, we define

$$
\sigma\left(\gamma_{i, j}\right):=\left(\sigma\left(b_{n}\right), \epsilon\right)
$$

which is nothing but the image of $b_{n} \in \widehat{B}_{n}$ by the $\widehat{G T}$-action on $\widehat{B}_{n}$ explained in $₫ 1$. 
(3) When $\gamma_{i, j}=c_{k, l}^{\epsilon}$, we define

$$
\sigma\left(\gamma_{i, j}\right):=f_{1 \cdots k, k+1, k+2}^{-1, t\left(\gamma_{i, j}\right)} \cdot \gamma_{i, j}
$$

with $f_{1 \cdots k, k+1, k+2}^{-1, t\left(\gamma_{i, j}\right)}=\left(f_{1 \cdots k, k+1, k+2}^{-1} \otimes e_{l}, t\left(\gamma_{i, j}\right)\right) \in \widehat{B}$. Figure 2.24 depicts the action.

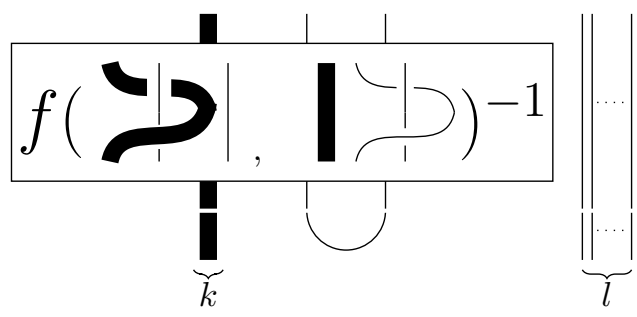

FIGURE 2.24. $\sigma\left(c_{k, l}^{\epsilon}\right)$

The symbol $\Lambda_{f}$ represents the profinite tangle

$$
a_{0,0}^{\curvearrowleft} \cdot a_{0,2}^{\curvearrowleft \downarrow \uparrow} \cdot\left(e_{1}^{\downarrow} \otimes f\right) \cdot c_{1,1}^{\downarrow \uparrow \uparrow} \cdot c_{0,0}^{\bigcup \uparrow}
$$

(cf. Figure 2.25).

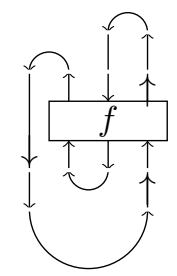

Figure 2.25. $\Lambda_{f}$

The symbol $\alpha(r)$ (resp. $\alpha(s)$ ) means the number of annihilations; the cardinality of the set $\left\{j \mid \gamma_{i, j} \in A\right\}$ for $i=1$ (resp. $i=2$ ) and $\left(\Lambda_{f}\right)^{\sharp \alpha(r)}\left(\operatorname{resp} .\left(\Lambda_{f}\right)^{\sharp \alpha(s)}\right)$ means the $\alpha(r)$-th (resp. the $\alpha(s)$-th ) power of $\Lambda_{f}$ with respect to $\sharp$. Particularly we have

$$
\sigma(\circlearrowleft) \sharp \Lambda_{f}=\circlearrowleft \in \operatorname{Frac} \widehat{\mathcal{K}}
$$

Proposition 2.40. Let $\sigma=(\lambda, f) \in \widehat{G T}$.

(1) If $r=\gamma_{1, m} \cdots \gamma_{1,2} \cdot \gamma_{1,1}\left(\gamma_{1, j}\right.$ : a profinite fundamental tangle) is a profinite knot, then $\left\{\sigma\left(\gamma_{1, m}\right) \cdots \sigma\left(\gamma_{1,2}\right) \cdot \sigma\left(\gamma_{1,1}\right)\right\}$ is again a profinite knot.

(2) The profinite tangle $\Lambda_{f}$ (Figure 2.25) is a profinite knot.

Proof. (1). When $\gamma=b_{n}^{\epsilon}$, since the projection

$$
p: \widehat{B}_{n} \rightarrow \mathfrak{S}_{n}
$$

is $\widehat{G T}$-equivalent (the action on $\mathfrak{S}_{n}$ means the trivial action), the skeleton never change, i.e. $\mathbb{S}(\sigma(\gamma))=\mathbb{S}(\gamma)$.

When $\gamma=a_{k, l}^{\epsilon}$ (resp. $c_{k, l}^{\epsilon}$ ), the skeleton $\mathbb{S}(\sigma(\gamma))$ is obtained by connecting $k+l+2$ straight bars on the top (resp. bottom) of $\mathbb{S}(\gamma)$ because $f \in \widehat{F}_{2} \subset \widehat{P}_{3}$. 
Therefore $\left\{\sigma\left(\gamma_{1, m}\right) \cdots \sigma\left(\gamma_{1,2}\right) \cdot \sigma\left(\gamma_{1,1}\right)\right\}$ is again a profinite knot.

(2). By Figure 2.25, it is easy because $f \in \widehat{F}_{2} \subset \widehat{P}_{3}$.

Theorem 2.41. The equation (2.19) determines a well-defined $\widehat{G T}$-action on Frac $\widehat{\mathcal{K}}$. Namely,

(1). $\sigma\left(\frac{r_{1}}{s_{1}}\right)=\sigma\left(\frac{r_{2}}{s_{2}}\right) \in \operatorname{Frac} \widehat{\mathcal{K}}$ if $r_{1} \sim r_{2}$ and $s_{1} \sim s_{2}$, i.e. if $r_{1}=r_{2}$ and $s_{1}=s_{2}$ in $\widehat{\mathcal{K}}$.

(2). $\sigma\left(\frac{r_{1}}{s_{1}}\right)=\sigma\left(\frac{r_{2}}{s_{2}}\right) \in \operatorname{Frac} \widehat{\mathcal{K}}$ if $\left(r_{1}, s_{1}\right) \approx\left(r_{2}, s_{2}\right)$, i.e. if $\frac{r_{1}}{s_{1}}=\frac{r_{2}}{s_{2}}$ in Frac $\widehat{\mathcal{K}}$.

(3). $\sigma_{1}\left(\sigma_{2}(x)\right)=\left(\sigma_{1} \circ \sigma_{2}\right)(x)$ for any $\sigma_{1}, \sigma_{2} \in \widehat{G T}$ and $x \in$ Frac $\widehat{\mathcal{K}}$.

Furthermore Frac $\widehat{\mathcal{K}}$ forms a topological $\widehat{G T}$-module. Namely,

(4). the action is compatible with the group structure, i.e.

$$
\sigma(e)=e, \quad \sigma(x \sharp y)=\sigma(x) \sharp \sigma(y), \quad \sigma\left(x^{-1}\right)=\sigma(x)^{-1}
$$

for any $\sigma \in \widehat{G T}$ and $x, y \in$ Frac $\widehat{\mathcal{K}}$.

(5). the action is continuous.

Proof. (1). Firstly we prove that $\sigma\left(\left(r_{1}, s\right)\right)=\sigma\left(\left(r_{2}, s\right)\right) \in \widehat{\mathcal{K}}^{2}$ when $r_{1}$ is isotopic to $r_{2}$ for $\sigma=(\lambda, f) \in \widehat{G T}$. We may further assume that $r_{1}$ is obtained from $r_{2}$ by a single operation of one of the moves (T1)-(T6).

- If it is (T1), it is clear.

- If it is (T2), it is immediate because $\sigma\left(b_{2}\right) \cdot \sigma\left(b_{1}\right)=\sigma\left(b_{2} b_{1}\right)$ holds for $b_{1}, b_{2} \in \widehat{B}_{n}$.

- If it is (T3), we may further assume that its $T_{1}$ and $T_{2}$ in (T3) are both fundamental profinite tangles. Then by Proposition 1.16 and Proposition 2.42

$$
\begin{aligned}
\sigma\left(e_{n_{1}}^{t\left(T_{1}\right)} \otimes T_{2}\right) & \cdot \sigma\left(T_{1} \otimes e_{m_{2}}^{s\left(T_{2}\right)}\right) \\
& =f_{\left[n_{1}\right],\left[n_{2}\right],[0]}^{\left.-1, t\left(T_{1}\right), t\left(T_{2}\right)\right)} \cdot\left(e_{n_{1}}^{t\left(T_{1}\right)} \otimes \sigma\left(T_{2}\right)\right) \cdot f_{\left[n_{1}\right],\left[m_{2}\right],[0]}^{\left(t\left(T_{1}\right), s\left(T_{2}\right)\right)} \cdot\left(\sigma\left(T_{1}\right) \otimes e_{m_{2}}^{s\left(T_{2}\right)}\right),
\end{aligned}
$$

by $(\mathrm{T} 4)$

$$
\begin{aligned}
& =f_{\left[n_{1}\right],\left[n_{2}\right],[0]}^{\left.-1, t\left(T_{2}\right)\right)} \cdot\left(e_{n_{1}}^{t\left(T_{1}\right)} \otimes \sigma\left(T_{2}\right)\right) \cdot\left(\sigma\left(T_{1}\right) \otimes e_{m_{2}}^{s\left(T_{2}\right)}\right) \cdot f_{\left[m_{1}\right],\left[m_{2}\right],[0]}^{\left.s\left(T_{1}\right), s\left(T_{2}\right)\right)} \\
& =f_{\left[n_{1}\right],\left[\left(T_{1}\right),[0]\right.}^{\left.-1\left(T_{2}\right)\right)} \cdot\left(\sigma\left(T_{1}\right) \otimes e_{n_{2}}^{t\left(T_{2}\right)}\right) \cdot\left(e_{m_{1}}^{s\left(T_{1}\right)} \otimes \sigma\left(T_{2}\right)\right) \cdot f_{\left[m_{1}\right],\left[m_{2}\right],[0]}^{\left.s\left(T_{2}\right)\right)} \\
& =\left(\sigma\left(T_{1}\right) \otimes e_{n_{2}}^{t\left(T_{2}\right)}\right) \cdot f_{\left[m_{1}\right],\left[n_{2}\right],[0]}^{-1,\left(s\left(T_{1}\right), t\left(T_{2}\right)\right)} \cdot\left(e_{m_{1}}^{s\left(T_{1}\right)} \otimes \sigma\left(T_{2}\right)\right) \cdot f_{\left[m_{1}\right],\left[m_{2}\right],[0]}^{\left(s\left(T_{1}\right), s\left(T_{2}\right)\right)} \\
& =\sigma\left(T_{1} \otimes e_{n_{2}}^{t\left(T_{2}\right)}\right) \cdot \sigma\left(e_{m_{1}}^{s\left(T_{1}\right)} \otimes T_{2}\right) .
\end{aligned}
$$

Whence (T3) is preserved by the $\widehat{G T}$-action.

- If it is (T4), we may assume that $T$ in (T4) is a fundamental profinite tangle. Then by Proposition 1.18 and Proposition 2.42

$$
\begin{aligned}
& \sigma\left(\mathrm{ev}_{k, t(T)}\left(b_{l}^{\epsilon}\right)\right) \cdot \sigma\left(e_{k-1}^{s_{1}} \otimes T \otimes e_{l-k}^{s_{2}}\right) \\
& =f_{\left[k^{\prime}-1\right],[n],\left[l-k^{\prime}\right]}^{-1,\left(t_{1}, t(T), t_{2}\right)} \cdot \mathrm{ev}_{k, t(T)}\left(\sigma\left(b_{l}^{\epsilon}\right)\right) \cdot f_{[k-1],[n],[l-k]}^{\left(s_{1}, t(T), s_{2}\right)} \\
& \cdot f_{[k-1],[n],[l-k]}^{-1,\left(s_{1}, t(T), s_{2}\right)} \cdot\left(e_{k-1}^{s_{1}} \otimes \sigma(T) \otimes e_{l-k}^{s_{2}}\right) \cdot f_{[k-1],[m],[l-k]}^{\left(s_{1}, s(T), s_{2}\right)} \\
& =f_{\left[k^{\prime}-1\right],[n],\left[l-k^{\prime}\right]}^{\left.-1, t_{1}, t(T), t_{2}\right)} \cdot \operatorname{ev}_{k, t(T)}\left(\sigma\left(b_{l}^{\epsilon}\right)\right) \cdot\left(e_{k-1}^{s_{1}} \otimes \sigma(T) \otimes e_{l-k}^{s_{2}}\right) \cdot f_{[k-1],[m],[l-k]}^{\left(s_{1}, s(T), s_{2}\right)},
\end{aligned}
$$


by $(\mathrm{T} 4)$

$$
\begin{aligned}
= & f_{\left[k^{\prime}-1\right],[n],\left[l-k^{\prime}\right]}^{-1,\left(t_{1}, t(T), t_{2}\right)} \cdot\left(e_{k^{\prime}-1}^{t_{1}} \otimes \sigma(T) \otimes e_{l-k^{\prime}}^{t_{2}}\right) \cdot \mathrm{ev}^{k^{\prime}, s(T)}\left(\sigma\left(b_{l}^{\epsilon}\right)\right) \cdot f_{[k-1],[m],[l-k]}^{\left(s_{1}, s(T), s_{2}\right)}, \\
= & f_{\left[k^{\prime}-1\right],\left[(T],\left[t_{2}\right)\right.}^{-1,\left(t_{1}, t(T), k^{\prime}\right]} \cdot\left(e_{k^{\prime}-1}^{t_{1}} \otimes \sigma(T) \otimes e_{l-k^{\prime}}^{t_{2}}\right) \cdot f_{\left[k^{\prime}-1\right],[m],\left[l-k^{\prime}\right]}^{\left(t_{1}, s(T), t_{2}\right.} \\
& \quad \cdot f_{\left[k^{\prime}-1\right],\left[(T),\left[t_{2}\right.\right.}^{-1,\left(t_{1}, k^{\prime}\right]} \cdot \mathrm{ev}^{k^{\prime}, s(T)}\left(\sigma\left(b_{l}^{\epsilon}\right)\right) \cdot f_{[k-1],[m],[l-k]}^{\left(s_{1}, s(T), s_{2}\right)} \\
= & \sigma\left(e_{k^{\prime}-1}^{t_{1}} \otimes T \otimes e_{l-k^{\prime}}^{t_{2}}\right) \cdot \sigma\left(\mathrm{ev}^{k^{\prime}, s(T)}\left(b_{l}^{\epsilon}\right)\right) .
\end{aligned}
$$

Whence (T4) is preserved by the action.

- If it is (T5), we have

$$
\sigma\left(a_{k+1, l-1}^{\epsilon^{\prime}}\right) \cdot \sigma\left(c_{k, l}^{\epsilon}\right)=a_{k+1, l-1}^{\epsilon^{\prime}} \cdot f_{1 \cdots k+1, k+2, k+3}^{s\left(c_{k, l}^{\epsilon}\right)} \cdot f_{1 \ldots k, k+1, k+2}^{-1, s\left(c_{k, l}^{\epsilon}\right)} \cdot c_{k, l}^{\epsilon} .
$$

By the pentagon equation (1.4)

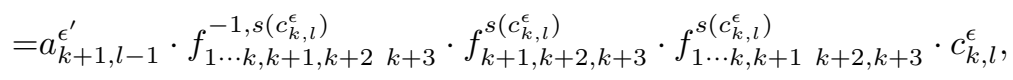

by (T4) and Lemma 1.8

$$
=a_{k+1, l-1}^{\epsilon^{\prime}} \cdot f_{k+1, k+2, k+3}^{s\left(c_{k, l}^{\epsilon}\right)} \cdot c_{k, l}^{\epsilon} .
$$

It looks that (T5) is not preserved by the $\widehat{G T}$-action. But actually it means that $\sigma\left(r_{1}\right)$ is obtained by an insertion of $f_{k+1, k+2, k+3}^{s\left(c_{k, l}^{\epsilon}\right)}$ between $a_{k+1, l-1}^{\epsilon^{\prime}}$ and $c_{k, l}^{\epsilon}$ in $\sigma\left(r_{2}\right)$. Thus $\sigma\left(r_{1}\right)=\sigma\left(r_{2}\right) \sharp \Lambda_{f}$. Because $\alpha\left(r_{1}\right)=\alpha\left(r_{2}\right)+1$, we may say that (T5) is compatible with the action by (2.19). The second equality can be proved in the same way.

- If it is (T6), again by Proposition 1.16 and Proposition 2.42

$$
\begin{aligned}
\sigma\left(a_{k, l}^{\epsilon}\right) & \cdot \sigma\left(\left(\sigma_{k+1}^{\epsilon^{\prime}}\right)^{c}\right) \\
& =a_{k, l}^{\epsilon} \cdot f_{1 \cdots k, k+1, k+2}^{s\left(a_{k, l}^{\epsilon}\right)} \cdot f_{1 \ldots k, k+1, k+2}^{-1, s\left(a_{k, l}^{\epsilon}\right)} \cdot\left(\sigma_{k+1}^{\epsilon^{\prime}}\right)^{\lambda c} \cdot f_{1 \ldots k, k+1, k+2}^{s\left(a_{k, l}^{\bar{\epsilon}}\right)} \\
& =a_{k, l}^{\epsilon} \cdot\left(\sigma_{k+1}^{\epsilon^{\prime}}\right)^{\lambda c} \cdot f_{1 \cdots k, k+1, k+2}^{s\left(a_{k, l}^{\epsilon}\right)},
\end{aligned}
$$

by $\lambda \equiv 1 \quad(\bmod 2)$ and $(\mathrm{T} 6)$

$$
=a_{k, l}^{\bar{\epsilon}} \cdot f_{1 \ldots k, k+1, k+2}^{s\left(a_{k, l}^{\bar{\epsilon}}\right)}=\sigma\left(a_{k, l}^{\bar{\epsilon}}\right) .
$$

The case for $c_{k, l}^{\epsilon}$ can be checked in the same way.

Secondly we prove that $\sigma\left(\left(r, s_{1}\right)\right)=\sigma\left(\left(r, s_{2}\right)\right) \in \widehat{\mathcal{K}}^{2}$ when $s_{1}$ is isotopic to $s_{2}$. But it can be proved in a similar way to the above. Hence our claim of (1) is obtained.

(2). By definition,

$$
r_{1} \sharp s_{2} \sharp t=r_{2} \sharp s_{1} \sharp t
$$

in $\mathcal{K}$ for some profinite knot $t$. By the definition of $\sharp$,

$$
\sigma\left(k_{1} \sharp k_{2}\right) \sharp \sigma(\bigcirc)=\sigma\left(k_{1}\right) \sharp \sigma\left(k_{2}\right)
$$

equivalently

$$
\sigma\left(k_{1} \sharp k_{2}\right)=\sigma\left(k_{1}\right) \sharp \sigma\left(k_{2}\right) \sharp \Lambda_{f},
$$


HIDEKAZU FURUSHO

holds in Frac $\widehat{\mathcal{K}}$ for any profinite knot $k_{1}$ and $k_{2}$. Therefore our claim is immediate because

and

$$
\sigma\left(r_{1} \sharp s_{2} \sharp t\right)=\sigma\left(r_{1}\right) \sharp \sigma\left(s_{2}\right) \sharp \sigma(t) \sharp \Lambda_{f} \sharp \Lambda_{f}
$$

$$
\sigma\left(r_{2} \sharp s_{1} \sharp t\right)=\sigma\left(r_{2}\right) \sharp \sigma\left(s_{1}\right) \sharp \sigma(t) \sharp \Lambda_{f} \sharp \Lambda_{f} .
$$

(3). For $\sigma_{1}=\left(\lambda_{1}, f_{1}\right)$ and $\sigma_{2}=\left(\lambda_{2}, f_{2}\right) \in \widehat{G T}$, put $\sigma_{3}=\sigma_{2} \circ \sigma_{1} \in \widehat{G T}$. Hence, by (1.5), $\sigma_{3}=\left(\lambda_{3}, f_{3}\right)$ with $\lambda_{3}=\lambda_{2} \lambda_{1}$ and

$$
f_{3}=f_{2} \cdot \sigma_{2}\left(f_{1}\right)=f_{2} \cdot f_{1}\left(x^{\lambda_{2}}, f_{2}^{-1} y^{\lambda_{2}} f_{2}\right)\left(=: f_{2} \circ f_{1}\right) .
$$

Firstly we note that

$$
\sigma_{3}\left(\gamma_{i, j}\right)=\sigma_{2}\left(\sigma_{1}\left(\gamma_{i, j}\right)\right) .
$$

When $\gamma_{i, j}=a_{k, l}^{\epsilon}$ or $c_{k, l}^{\epsilon}$, the equality is derived from (2.23). When $\gamma_{i, j}=b_{n}^{\epsilon}$, it is easy because of the $\widehat{G T}$-action on $\widehat{B}_{n}$.

Secondly by definition we have

$\sigma_{2}\left(\Lambda_{f_{1}}\right)=\left\{\sigma_{2}\left(a_{0,0}\right) \cdot \sigma_{2}\left(a_{0,2}\right) \cdot \sigma_{2}\left(e_{1} \otimes f_{1}\right) \cdot \sigma_{2}\left(c_{1,1}\right) \cdot \sigma_{2}\left(c_{0,0}\right)\right\} / \Lambda_{f_{2}}^{\sharp 2}$.

By Proposition 1.16 and Proposition 2.42

$$
\begin{aligned}
&=\left\{a_{0,0} \cdot a_{0,2} \cdot\left(f_{2}^{-1}\right)_{1,2,3} \cdot\left(f_{2}^{-1}\right)_{1,23,4} \cdot\left(e_{1} \otimes \sigma_{2}\left(f_{1}\right)\right)\right. \\
&\left.\cdot\left(f_{2}\right)_{1,23,4} \cdot\left(f_{2}\right)_{1,2,3} \cdot\left(f_{2}^{-1}\right)_{1,2,3} \cdot c_{1,1} \cdot c_{0,0}\right\} / \Lambda_{f_{2}}^{\sharp 2} \\
&=\left\{a_{0,0} \cdot a_{0,2} \cdot\left(f_{2}^{-1}\right)_{1,2,3} \cdot\left(f_{2}^{-1}\right)_{1,23,4} \cdot\left(e_{1} \otimes \sigma_{2}\left(f_{1}\right)\right) \cdot\left(f_{2}\right)_{1,23,4} \cdot c_{1,1} \cdot c_{0,0}\right\} / \Lambda_{f_{2}}^{\sharp 2},
\end{aligned}
$$

by the pentagon equation (1.4)

$$
\begin{aligned}
=\left\{a_{0,0} \cdot a_{0,2} \cdot\left(f_{2}^{-1}\right)_{12,3,4} \cdot\left(f_{2}^{-1}\right)_{1,2,34} \cdot\left(f_{2}\right)_{2,3,4} \cdot\left(e_{1} \otimes \sigma_{2}\left(f_{1}\right)\right)\right. \\
\\
\left.\cdot\left(f_{2}\right)_{1,23,4} \cdot c_{1,1} \cdot c_{0,0}\right\} / \Lambda_{f_{2}}^{\sharp 2},
\end{aligned}
$$

by a successive application of (T6) and Lemma 1.8

$$
\begin{aligned}
& =\left\{a_{0,0} \cdot a_{0,2} \cdot\left(f_{2}\right)_{2,3,4} \cdot\left(e_{1} \otimes \sigma_{2}\left(f_{1}\right)\right) \cdot c_{1,1} \cdot c_{0,0}\right\} / \Lambda_{f_{2}}^{\sharp 2} \\
& =\left\{a_{0,0} \cdot a_{0,2} \cdot\left(e_{1} \otimes f_{2} \cdot \sigma_{2}\left(f_{1}\right)\right) \cdot c_{1,1} \cdot c_{0,0}\right\} / \Lambda_{f_{2}}^{\sharp 2} \\
& =\Lambda_{f_{2} \circ f_{1} \sharp \sigma_{2}(\circlearrowleft)^{\sharp 2} .}
\end{aligned}
$$

We note that in the above computation we omit the symbol $\epsilon$ of orientation.

Finally

$$
\begin{aligned}
\sigma_{2}\left(\sigma_{1}\left(\frac{r}{s}\right)\right)= & \sigma_{2}\left(\frac{\left\{\sigma_{1}\left(\gamma_{1, m}\right) \cdots \sigma_{1}\left(\gamma_{1,2}\right) \cdot \sigma_{1}\left(\gamma_{1,1}\right)\right\} \sharp\left(\Lambda_{f_{1}}\right)^{\sharp \alpha(s)}}{\left\{\sigma_{1}\left(\gamma_{2, n}\right) \cdots \sigma_{1}\left(\gamma_{2,2}\right) \cdot \sigma_{1}\left(\gamma_{2,1}\right)\right\} \sharp\left(\Lambda_{f_{1}}\right)^{\sharp \alpha(r)}}\right) \\
= & \frac{\sigma_{2}\left(\left\{\sigma_{1}\left(\gamma_{1, m}\right) \cdots \sigma_{1}\left(\gamma_{1,2}\right) \cdot \sigma_{1}\left(\gamma_{1,1}\right)\right\}\right) \sharp \sigma_{2}\left(\Lambda_{f_{1}}\right)^{\sharp \alpha(s)} \sharp \Lambda_{f_{2}}^{\sharp \alpha(s)}}{\sigma_{2}\left(\left\{\sigma_{1}\left(\gamma_{2, n}\right) \cdots \sigma_{1}\left(\gamma_{2,2}\right) \cdot \sigma_{2}\left(\gamma_{2,1}\right)\right\}\right) \sharp \sigma_{2}\left(\Lambda_{f_{1}}\right)^{\sharp \alpha(r)} \sharp \Lambda_{f_{2}}^{\sharp \alpha(r)}} \\
= & \frac{\left\{\sigma_{2}\left(\sigma_{1}\left(\gamma_{1, m}\right)\right) \cdots \sigma_{2}\left(\sigma_{1}\left(\gamma_{1,1}\right)\right)\right\} \sharp \Lambda_{f_{2}}^{\sharp \alpha(s)} \sharp\left(\Lambda_{f_{3}}\right)^{\sharp \alpha(s)} \sharp \sigma_{2}(\bigcirc)^{\sharp 2 \alpha(s)} \sharp \Lambda_{f_{2}}^{\sharp \alpha(s)}}{\left\{\sigma_{2}\left(\sigma_{1}\left(\gamma_{2, n}\right)\right) \cdots \sigma_{2}\left(\sigma_{1}\left(\gamma_{2,1}\right)\right)\right\} \sharp \Lambda_{f_{2}}^{\sharp \alpha(r)} \sharp\left(\Lambda_{f_{3}}\right) \sharp \alpha(r) \sharp \sigma_{2}(\circlearrowleft)^{\sharp 2 \alpha(r)} \sharp \Lambda_{f_{2}}^{\sharp \alpha(r)}} \\
= & \frac{\left\{\sigma_{3}\left(\gamma_{1, m}\right) \cdots \sigma_{3}\left(\gamma_{1,2}\right) \cdot \sigma_{3}\left(\gamma_{1,1}\right)\right\} \sharp\left(\Lambda_{f_{3}}\right)^{\sharp \alpha(s)}}{\left\{\sigma_{3}\left(\gamma_{2, n}\right) \cdots \sigma_{3}\left(\gamma_{2,2}\right) \cdot \sigma_{3}\left(\gamma_{2,1}\right)\right\} \sharp\left(\Lambda_{f_{3}}\right)^{\sharp \alpha(r)}}=\sigma_{3}\left(\frac{r}{s}\right)
\end{aligned}
$$

by (2.20) and (2.22). 
(4). Let $x=r_{1} / s_{1}$ and $y=r_{2} / s_{2}$ with profinite knots $r_{1}, r_{2}, s_{1}, s_{2}$. Then by (2.22) it is easy to see

$$
\begin{aligned}
\sigma(x \sharp y) & =\sigma\left(\frac{r_{1} \sharp r_{2}}{s_{1} \sharp s_{2}}\right)=\frac{\sigma\left(r_{1} \sharp r_{2}\right)}{\sigma\left(s_{1} \sharp s_{2}\right)}=\frac{\sigma\left(r_{1}\right) \sharp \sigma\left(r_{2}\right) \sharp \Lambda_{f}}{\sigma\left(s_{1}\right) \sharp \sigma\left(s_{2}\right) \sharp \Lambda_{f}}=\frac{\sigma\left(r_{1}\right) \sharp \sigma\left(r_{2}\right)}{\sigma\left(s_{1}\right) \sharp \sigma\left(s_{2}\right)} \\
& =\frac{\sigma\left(r_{1}\right)}{\sigma\left(s_{1}\right)} \sharp \frac{\sigma\left(r_{2}\right)}{\sigma\left(s_{2}\right)}=\sigma\left(\frac{r_{1}}{s_{1}}\right) \sharp \sigma\left(\frac{r_{2}}{s_{2}}\right)=\sigma(x) \sharp \sigma(y) .
\end{aligned}
$$

The inverse is also easy to check.

(5). We recall that $\widehat{\mathcal{K}}^{\text {seq }}$ (cf. the proof of Theorem 2.16, (2)) is the set of finite consistent sequences of profinite fundamental tangles $\gamma_{n} \cdots \gamma_{2} \cdot \gamma_{1}$ with a single connected component and with $\left(\gamma_{n}, \gamma_{1}\right)=(\curvearrowleft, \cup)$. We define the map

$$
A: \widehat{G T} \times{\widehat{\mathcal{K}^{\prime}}}^{\text {seq }} \times \widehat{\mathcal{K}}^{\text {seq }} \rightarrow \widehat{\mathcal{K}}^{\text {seq }} \times{\widehat{\mathcal{K}^{\prime}}}^{\text {seq }}
$$

by

$A(\sigma, r, s)=\left(\left\{\sigma\left(\gamma_{1, m}\right) \cdots \sigma\left(\gamma_{1,2}\right) \cdot \sigma\left(\gamma_{1,1}\right)\right\} \sharp\left(\Lambda_{f}\right)^{\sharp \alpha(s)},\left\{\sigma\left(\gamma_{2, n}\right) \cdots \sigma\left(\gamma_{2,2}\right) \cdot \sigma\left(\gamma_{2,1}\right)\right\} \sharp\left(\Lambda_{f}\right)^{\sharp \alpha(r)}\right)$

for $\sigma=(\lambda, f), r=\gamma_{1, m} \cdots \gamma_{1,2} \cdot \gamma_{1,1}$ and $s=\gamma_{2, n} \cdots \gamma_{2,2} \cdot \gamma_{2,1}\left(\gamma_{i, j}\right.$ : profinite fundamental tangle). We know that the $\widehat{G T}$-action on $\widehat{B}_{n}$ and the map $\widehat{G T} \rightarrow \widehat{B}_{3}$ : $\sigma=(\lambda, f) \mapsto f$ are continuous, so the map $A$ is continuous. Since the diagram below is commutative

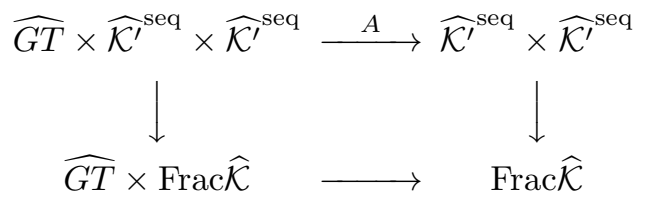

and the projection $\widehat{\mathcal{K}}^{\text {seq }} \times \widehat{\mathcal{K}}^{\text {seq }} \rightarrow$ Frac $\widehat{\mathcal{K}}$ is continuous, the lower map is also continuous.

The following is required to prove Theorem 2.41.

Proposition 2.42. Let $k, l, m_{1}, m_{2} \geqslant 0$ and $\epsilon_{i} \in\{\uparrow, \downarrow\}^{m_{i}}(i=1,2)$. For any $\sigma \in \widehat{G T}$,

$$
\sigma\left(a_{m_{1}+k, l+m_{2}}^{\epsilon_{1} \epsilon_{2}}\right)=f_{\left[m_{1}\right],[k+l],\left[m_{2}\right]}^{-1, \epsilon_{t}} \cdot\left(e_{m_{1}}^{\epsilon_{1}} \otimes \sigma\left(a_{k, l}^{\epsilon}\right) \otimes e_{m_{2}}^{\epsilon_{2}}\right) \cdot f_{\left[m_{1}\right],[k+l+2],\left[m_{2}\right]}^{\epsilon_{s}}
$$

with $\epsilon_{t}=t\left(a_{m_{1}+k, l+m_{2}}^{\epsilon_{1} \epsilon_{2}}\right)$ and $\epsilon_{s}=s\left(a_{m_{1}+k, l+m_{2}}^{\epsilon_{1} \epsilon \epsilon_{2}}\right)$. And

$$
\sigma\left(c_{m_{1}+k, l+m_{2}}^{\epsilon_{1} \epsilon_{2}}\right)=f_{\left[m_{1}\right],[k+l+2],\left[m_{2}\right]}^{-1, \epsilon_{t}} \cdot\left(e_{m_{1}}^{\epsilon_{1}} \otimes \sigma\left(c_{k, l}^{\epsilon}\right) \otimes e_{m_{2}}^{\epsilon_{2}}\right) \cdot f_{\left[m_{1}\right],[k+l],\left[m_{2}\right]}^{\epsilon_{s}}
$$

with $\epsilon_{t}=t\left(a_{m_{1}+k, l+m_{2}}^{\epsilon_{1} \epsilon \epsilon_{2}}\right)$ and $\epsilon_{s}=s\left(a_{m_{1}+k, l+m_{2}}^{\epsilon_{1} \epsilon \epsilon_{2}}\right)$.

Here $f_{\left[m_{1}\right],[M],\left[m_{2}\right]}^{\epsilon} \in \widehat{B}$ means $\left(f_{\left[m_{1}\right],[M],\left[m_{2}\right]}, \epsilon\right) \in \widehat{B}_{m_{1}+M+m_{2}} \times\{\uparrow, \downarrow\}^{m_{1}+M+m_{2}}$ with (see also (1.13)

$$
\begin{gathered}
f_{\left[m_{1}\right],[M],\left[m_{2}\right]}:=f_{1 \cdots m_{1}, m_{1}+1 \cdots m_{1}+M-1, m_{1}+M} \cdot f_{1 \cdots m_{1}, m_{1}+1 \cdots m_{1}+M-2, m_{1}+M-1} \\
\cdots f_{1 \cdots m_{1}, m_{1}+1, m_{1}+2} \in \widehat{B}_{m_{1}+M+m_{2}} .
\end{gathered}
$$

Proof. We prove the first equality. To avoid the complexity, we again omit the symbol of orientations. By Definition 2.39,(1), 


$$
\begin{aligned}
& f_{\left[m_{1}\right],[k+l],\left[m_{2}\right]}^{-1} \cdot\left(e_{m_{1}} \otimes \sigma\left(a_{k, l}\right) \otimes e_{m_{2}}\right) \cdot f_{\left[m_{1}\right],[k+l+2],\left[m_{2}\right]} \\
& \quad=f_{\left[m_{1}\right],[k+l],\left[m_{2}\right]}^{-1} \cdot a_{m_{1}+k, l+m_{2}} \cdot f_{m_{1}+1 \cdots m_{1}+k, m_{1}+k+1, m_{1}+k+2} \cdot f_{\left[m_{1}\right],[k+l+2],\left[m_{2}\right]} .
\end{aligned}
$$

- When $M \geqslant k+3$, by (T4),

$f_{m_{1}+1 \cdots m_{1}+k, m_{1}+k+1, m_{1}+k+2}$ commutes with $f_{1 \cdots m_{1}, m_{1}+1 \cdots m_{1}+M-1, m_{1}+M}$ and

$a_{m_{1}+k, l+m_{2}} \cdot f_{1 \cdots m_{1}, m_{1}+1 \cdots m_{1}+M-1, m_{1}+M}=f_{1 \cdots m_{1}, m_{1}+1 \cdots m_{1}+M-3, m_{1}+M-2} \cdot a_{m_{1}+k, l+m_{2}}$.

Therefore

(2.24) $=f_{\left[m_{1}\right],[k],\left[l+m_{2}\right]}^{-1} \cdot a_{m_{1}+k, l+m_{2}} \cdot f_{m_{1}+1 \cdots m_{1}+k, m_{1}+k+1, m_{1}+k+2} \cdot f_{\left[m_{1}\right],[k+2],\left[l+m_{2}\right]}$.

- When $M=k+1, k+2$, our calculation goes as follows.

(2.24) $=f_{\left[m_{1}\right],[k],\left[l+m_{2}\right]}^{-1} \cdot a_{m_{1}+k, l+m_{2}} \cdot f_{m_{1}+1 \cdots m_{1}+k, m_{1}+k+1, m_{1}+k+2}$.

$f_{1 \cdots m_{1}, m_{1}+1 \cdots m_{1}+k+1, m_{1}+k+2} \cdot f_{1 \cdots m_{1}, m_{1}+1 \cdots m_{1}+k, m_{1}+k+1} \cdot f_{\left[m_{1}\right],[k],\left[l+m_{2}+2\right]}$,

by the pentagon equation (1.4),

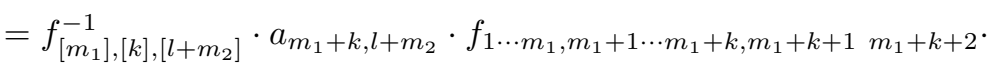

$f_{1 \cdots m_{1}+k, m_{1}+k+1, m_{1}+k+2} \cdot f_{\left[m_{1}\right],[k],\left[l+m_{2}+2\right]}$,

by (T4) and Lemma 1.8 .

$=f_{\left[m_{1}\right],[k],\left[l+m_{2}\right]}^{-1} \cdot a_{m_{1}+k, l+m_{2}} \cdot f_{1 \cdots m_{1}+k, m_{1}+k+1, m_{1}+k+2} \cdot f_{\left[m_{1}\right],[k],\left[l+m_{2}+2\right]} \cdot$

- When $M \leqslant k$, by (T4) again,

$f_{1 \cdots m_{1}+k, m_{1}+k+1, m_{1}+k+2}$ commutes with $f_{1 \cdots m_{1}, m_{1}+1 \cdots m_{1}+M-1, m_{1}+M}$ and

$a_{m_{1}+k, l+m_{2}} \cdot f_{1 \cdots m_{1}, m_{1}+1 \cdots m_{1}+M-1, m_{1}+M}=f_{1 \cdots m_{1}, m_{1}+1 \cdots m_{1}+M-1, m_{1}+M} \cdot a_{m_{1}+k, l+m_{2}}$.

Therefore

$$
\begin{aligned}
(2.24) & =f_{\left[m_{1}\right],[k],\left[l+m_{2}\right]}^{-1} \cdot a_{m_{1}+k, l+m_{2}} \cdot f_{\left[m_{1}\right],[k],\left[l+m_{2}+2\right]} \cdot f_{1 \cdots m_{1}+k, m_{1}+k+1, m_{1}+k+2}, \\
& =a_{m_{1}+k, l+m_{2}} \cdot f_{1 \cdots m_{1}+k, m_{1}+k+1, m_{1}+k+2}=\sigma\left(a_{m_{1}+k, l+m_{2}}\right) .
\end{aligned}
$$

Hence we get the equality.

The second equality can be proved in the same way.

Thus by Theorem 2.41, the $\widehat{G T}$-action

$$
\widehat{G T} \rightarrow \text { AutFrac } \widehat{\mathcal{K}}
$$

is established.

We note that due to the creation-annihilation relations (T5) we have to pass to the fractional group of $\widehat{\mathcal{K}}$ to construct $\widehat{G T}$-action on profinite knots.

Remark 2.43. In $[\mathrm{KT}]$, it is explained that the category $\widehat{\mathcal{T}}(R)(R$ : a commutative ring containing $\mathbb{Q}$ ) of pro- $R$-algebraic framed tangles forms an $R$-linear ribbon category. From which they deduced an action of the proalgebraic GrothendieckTeichmüller group $G T(R)$ Dr2] on the space $\widehat{R[\mathcal{K}]}$ of pro- $R$-algebraic knots by a categorical arguments. In our forthcoming paper [F2, it will be shown that our action (2.25) is a lift of their action. An analogous deduction of our Theorem 2.41 by such categorical arguments might be expectable. However a completely same argument does not seem to work. We may have a 'ribbon' category $\widehat{\mathcal{T}}$ of profinite 
(framed) tangles but a difficulty here is that the inverse $\left(\Lambda_{f}\right)^{-1}$ does not look to exist generally in $\widehat{\mathcal{T}}$, unlike the case of $\widehat{\mathcal{T}}(R)$. (That is why we introduced the group Frac $\widehat{\mathcal{K}}$ of the fraction of the monoid $\widehat{\mathcal{K}}$. A technical care to remedy this might be required.)

Remark 2.44. The Kontsevich knot invariant $\mathrm{KO}$ is obtained by integrating a formal analogue of the Knizhnik-Zamolodchikov (KZ) equation. Bar-Natan Ba1, Kassel-Turaev [KT], Le-Murakami [LM] and Pieunikhin [P] gave a combinatorial reconstruction of the invariant by using an associator Dr2. An associator means a pair $(\mu, \varphi)$ with $\mu \in R^{\times}$and an $R$-coefficient non-commutative formal power series $\varphi$ with two variables satisfying specific relations which are analogues of our pentagon and hexagon equations (1.4)-(1.3) (Dr2, see also [F1]). One of striking results 11 in Le-Murakami [LM] is the rationality of the Kontsevich invariant which follows from that the resulting invariant is, in fact, independent of $\varphi$ (but depends on $\mu$ ). Stimulated to their result, Kassel and Turaev [KT] showed that their $G T(R)$-action on $\widehat{R[\mathcal{K}]}$ factors through the cyclotomic action (cf. Appendix of $[\mathrm{KT}]$ ). The algebra $\widehat{R[\mathcal{K}]}$ looks 'too linear'. In contrast, in our profinite setting, it is totally unclear if our above $\widehat{G T}$-action (2.25) would depend only on $\lambda \in \widehat{\mathbb{Z}}^{\times}$of $(\lambda, f) \in \widehat{G T}$, namely, the action would factor through $\widehat{\mathbb{Z}}^{\times}$. We remind that their proof of the above independency is based on certain linear algebraic arguments, actually a vanishing of a (Harrison) cohomology of a chain complex associated with chords. But here in our situation, we are working not on their proalgebraic setting but on the profinite setting where such vanishing result has not been established. And we do not know whether such a factorization would occur in our setting or not.

Finally we obtain a Galois representation on knots as an important consequence of Theorem 2.41

Theorem 2.45. Fix an embedding from $\overline{\mathbb{Q}}$ in to $\mathbb{C}$. The group Frac $\widehat{\mathcal{K}}$ of profinite knots admits a non-trivial topological $G_{\mathbb{Q}}$-module structure. Namely there is a nontrivial continuous Galois representation

$$
\rho_{0}: G_{\mathbb{Q}} \rightarrow \text { Aut Frack } \widehat{\mathcal{K}}
$$

Proof. By Theorem 2.41 it is straightforward because in Theorem 1.9 we see that the absolute Galois group $G_{\mathbb{Q}}$ is mapped to $\widehat{G T}$. It is proved that Frac $\widehat{\mathcal{K}}$ is nontrivial in Theorem 2.36. The non-triviality of $\rho_{0}$ is a consequence of the example below because generally we have $K \neq \bar{K}$ in Frac $\widehat{\mathcal{K}}$ : For instance, the left trefoil (the knot in the left below of Figure 2.15) and the right trefoil (its mirror image) are mapped to different elements by the map (2.17) because they are known to be separated by the Kontsevich invariant. (cf. Remark 2.34.)

Example 2.46. Especially when $\sigma \in G_{\mathbb{Q}}$ is equal to the complex conjugation morphism $\varsigma_{0}$, it corresponds to $(\lambda, f)=(-1,1) \in \widehat{G T}$ whose action on $\widehat{B}_{n}$ is given by $\sigma_{i} \mapsto \sigma_{i}^{-1}(1 \leqslant i \leqslant n-1)$ (cf. Example1.10). Whence the action of $\varsigma_{0}$ on Frac $\widehat{\mathcal{K}}$ is particularly described by

$$
\rho_{0}\left(\varsigma_{0}\right)(K)=\bar{K}
$$

for $K \in \mathcal{K}$ because $\Lambda_{1}=0$. Here we denote the image of the map $h^{\prime}$ (2.18) on an oriented knot $K$ by the same symbol $K$ and we mean the mirror image of the knot

${ }^{11}$ It might be amazing to know that Drinfeld indicated it in Dr1]. 
$K$ by $\bar{K}$. The easiest example is that the right trefoil knot is mapped to the left trefoil knot by the complex conjugation.

There is another type of involution. For each profinite oriented knot $K$, we define $\operatorname{rev}(K)$ to be a profinite oriented knot which is obtained by reversing the orientation of $K$. It is easy to see that it induces a well-defined involution

$$
\text { rev }: \text { Frac } \widehat{\mathcal{K}} \rightarrow \text { Frac } \widehat{\mathcal{K}}
$$

which is an automorphism as a topological group.

Problem 2.47. Is rev defined over $\mathbb{Q}$ ? Namely does

$$
\text { rev } \circ \sigma=\sigma \circ \text { rev }
$$

hold for all $\sigma \in G_{\mathbb{Q}}$ ?

This problem would be proven affirmatively if we could show that $\Lambda_{f}=\operatorname{rev}\left(\Lambda_{f}\right)$ in Frac $\widehat{\mathcal{K}}$ for all $\sigma=(\lambda, f) \in G_{\mathbb{Q}}$. In the proalgebraic setting (cf. Remark 2.44 and [F2]), rev can be defined similarly for $\widehat{R[\mathcal{K}]}$. We can show the validity of an analogue of (2.27) for the action of $\sigma \in G T(R)$ on $\widehat{R[\mathcal{K}]}$ by transmitting the $G T(R)$-action on $\widehat{R[\mathcal{K}]}$ into a $G R T(R)$-action on $\widehat{C D}$.

Extending other standard operations on knots, such as mutation and cabling, into those on profinite knots and examining their Galois behaviors is also worthy to pursue.

Project 2.48. In $₫ 1$ the actions of $\widehat{G T}$ and $G_{\mathbb{Q}}$ on the profinite braid group $\widehat{B}_{n}$ are discussed. In Remark 1.14 it is explained in the language of algebraic geometry that the $G_{\mathbb{Q}}$-action on $\widehat{B}_{n}$ is caused by the homotopy exact sequence of the scheme-theoretic fundamental group of the quotient variety $\operatorname{Conf}_{\mathfrak{S}_{n}}^{n}$ of the configuration space $\operatorname{Conf}^{n}$. Whilst as for our Galois action on knots in Theorem 2.45, the author is not sure whether there is such kind of its 'purely' algebraicgeometrical interpretation (without a usage of $\widehat{G T}$-factorization) or not. In other word, it is not clear if there exists any (co)homology theory $H_{\star}$ (or any fundamental group theory $\pi_{1}^{\star}$ ) and any (pro-)variety $X$ defined over $\mathbb{Q}$ such that

$$
\operatorname{Frac} \widehat{\mathcal{K}}=H_{\star}\left(X_{\overline{\mathbb{Q}}}\right)
$$

(or Frac $\left.\widehat{\mathcal{K}}=\pi_{1}^{\star}\left(X_{\overline{\mathbb{Q}}}\right)\right)$ and the right hand side naturally carries a $G_{\mathbb{Q}^{-}}$action which

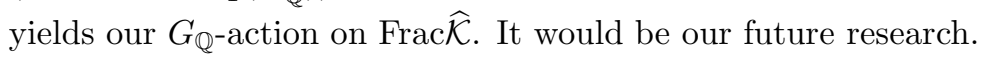

Asking the validity of an analogue of Belyı's theorem [Be] in (1.8) is particularly significant.

Problem 2.49. Is the action of the absolute Galois group (2.26) on profinite knots faithful?

If not, then what is the corresponding kernel field? And what is the arithmetic meaning of this?

This is also related to the problem discussed in Remark 2.44 above. In [F2, it will be explained that the corresponding kernel field is bigger than the maximal abelian extension $\mathbb{Q}\left(\mu_{\infty}\right)$ of $\mathbb{Q}$.

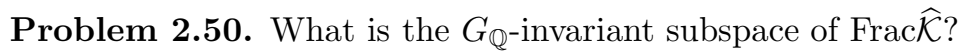


In [F2, we will settle a similar problem formulated in the proalgebraic setting. Asking the same type of questions for each given knot is also worthy to discuss.

Problem 2.51. (1). What is the Galois stabilizer of each given knot? And what is the corresponding Galois extension field of $\mathbb{Q}$ ?

(2). Suppose that two topological knots $K_{1}$ and $K_{2}$ and an open normal subgroup $\mathcal{N}$ of Frack $\widehat{\mathcal{K}}$ with finite index are given. When do two cosets $K_{1} \cdot \mathcal{N}$ and $K_{2} \cdot \mathcal{N}$ lie on a same Galois orbit? If so, then which $\sigma \in G_{\mathbb{Q}}$ connect them?

Example 2.46 tells that a knot and its mirror image lie on a same Galois orbit and that the stabilizer of an amphicheiral knot, say, the figure eight knot (the knot in the left above of Figure 2.15), contains the subgroup $\left\{i d, \varsigma_{0}\right\}$ of order 2.

Project 2.52. We can also consider Galois action not only on profinite knots but also on pro-solvable knots by replacing profinite pure braid groups $\hat{P}_{n}$ by their prosolvable completions on the definition of profinite knots. The direction might be also worthy to pursue. Ihara's profinite beta function $B_{\sigma}$ [1] which arises from a description of the action of the absolute Galois group on the double commutator quotient of $\widehat{F}_{2}$ might help to see the action on a certain quotient.

As is explained in Remark 2.34, we have an isomorphism $\widehat{\mathbb{Q}[\mathcal{K}]} \simeq \widehat{C D}$ between the linear space of pro-algebraic knots and that of chord diagrams.

Problem 2.53. What is a profinite analogue of the linear space of chord diagrams? Do we have a profinite analogue of the above isomorphism for $\widehat{\mathcal{K}}$ and this?

Grothendieck's dessins d'enfants G2 are combinatorial diagrams which describe the action of the absolute Galois group on $\widehat{F}_{2}$. The author wonders if a certain projective system of dessins attains the above profinite analogue of the space of chord diagrams.

Project 2.54. There are various notions of equivalences for (framed) knots (and links) such as the Kirby moves (the Fenn-Rourke moves), the knot cobordism, the knot concordance, etc. Extending these notions into those for our profinite links and examining their behaviors under our Galois action is worthy to pursue. Particularly the Kirby moves are known (consult the standard textbook such as [01]) to yield a one to one correspondence between the set of framed links modulo the equivalence generated by the moves and the set of isomorphism classes of closed connected oriented 3-manifolds (three dimensional manifolds) by Dehn surgery. Giving a nice formulation of profinite analogues of Kirby moves and a description of their Galois behaviors looks significant for a realization of the analogy between number rings and 3-manifolds, which is one of the most fundamental ones posted in arithmetic topology by Kapranov $\mathrm{Kap}$, Morishita $[\mathrm{Mo}$ and Reznikov $[\mathrm{R}$.

\section{Appendix A. Two-BRIDGe PROFInite Knots}

We introduce profinite analogues of two-bridge knots and observe that the subgroup of $G \widehat{\mathcal{K}}$ generated by them is stable under our Galois action.

We recall that a two-bridge knot (or link) is a topological knot (or link) which can be isotoped so that the natural height function given by the $z$-coordinate has only two maxima and two minima as critical points. 
Definition A.1. A profinite knot (resp. link) is called a two-bridge profinite knot (resp. link) when it is isotopic to the presentation $a_{0,0}^{\epsilon_{1}} \cdot a_{0,2}^{\epsilon_{2}} \cdot b_{4}^{\epsilon_{3}} \cdot c_{1,1}^{\epsilon_{4}} \cdot c_{0,0}^{\epsilon_{5}}$ with $b_{4} \in \widehat{B}_{4}$, which is depicted in Figure A.1 with its orientation ignored.

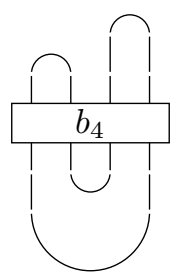

Figure A.1. Two-bridge profinite knot

We note that each isotopy class of two-bridge (topological) knot naturally determines an isotopy class of two-bridge profinite knot.

We recall that a two-bridge (topological) knot is also called as a rational knot because each isotopy class of two-bridge knot (or link) is characterized by a rational number via its continued fraction expansion (cf. [Lic]). As its profinite analogue, it is interesting to see if the set of isotopy classes of two-bridge profinite knots can be also parametrized by any numbers or not.

We denote the subgroup of $G \widehat{\mathcal{K}}$ generated by two-bridge profinite knots by $\mathcal{B} r(2)$.

Proposition A.2. The subgroup $\mathcal{B} r(2)$ is stable under our Galois action.

Proof. It can be verified by showing that $\mathcal{B} r(2)$ is stable by our $\widehat{G T}$-action. Let $\sigma=$ $(\lambda, f) \in \widehat{G T}$ and let $K$ be a two-bridge profinite knot with the above presentation. Then

$$
\begin{aligned}
\sigma\left(\frac{K}{\circlearrowleft}\right) & =\frac{\sigma(K)}{\sigma(\circlearrowleft)}=\frac{\left\{\sigma\left(a_{0,0}^{\epsilon_{1}}\right) \cdot \sigma\left(a_{0,2}^{\epsilon_{2}}\right) \cdot \sigma\left(b_{4}^{\epsilon_{3}}\right) \cdot \sigma\left(c_{1,1}^{\epsilon_{4}}\right) \cdot \sigma\left(c_{0,0}^{\epsilon_{5}}\right)\right\} \sharp \Lambda_{f}}{\left(\Lambda_{f}\right)^{\sharp 2}} \\
& =\frac{a_{0,0}^{\epsilon_{1}} \cdot a_{0,2}^{\epsilon_{2}} \cdot\left(\sigma\left(b_{4}^{\epsilon_{3}}\right) \cdot f_{123}^{-1}\right) \cdot c_{1,1}^{\epsilon_{4}} \cdot c_{0,0}^{\epsilon_{5}}}{\Lambda_{f}} .
\end{aligned}
$$

Since both numerator and denominator of the last term are two-bridge knots and $\widehat{G T}$ acts on $G \widehat{\mathcal{K}}$ as a group automorphism, our claim is obtained.

Considering profinite analogues of other types of (topological) knots such as hyperbolic knots, torus knots, etc. and considering their Galois behaviors might be interesting problems.

\section{Appendix B. Profinite fRAmed Knots}

We will extend our definition of profinite analogue of knots into that of framed knots and show that the absolute Galois group acts on the group generated by them. The construction is given in a same way to our arguments in $\$ 2$,

Definition B.1. The set $\widehat{\mathcal{F T}}$ of profinite framed tangles (isotopy classes of profinite framed tangle diagrams) is defined to be the set of profinite tangle diagrams divided by the framed isotopy, the equivalence given by a finite number of the moves (FT1)(FT6). Here (FT1)-(FT5) are same to (T1)-(T5) given in $\$ 2$ while (FT6) is given 
below. The set $\widehat{\mathcal{F K}}$ (resp. $\widehat{\mathcal{F L}}$ ) of profinite framed knots (resp. links) is the subset of $\widehat{\mathcal{F T}}$ which consists of framed isotopy classes of profinite knot (resp. link) diagrams.

(FT6) First framed Reidemeister move: for $c \in \widehat{\mathbb{Z}}, c_{k, l+2}^{\epsilon_{1}}, c_{k+2, l}^{\epsilon_{2}} \in C, a_{k+1, l+1}^{\epsilon_{3}} \in$ $A$ and $\sigma_{k+1}^{\epsilon_{1}^{\prime}}, \sigma_{k+3}^{\epsilon_{2}^{\prime}} \in \widehat{B}$ representing $\sigma_{k+1} \in \widehat{B}_{k+l+2}$ and $\sigma_{k+3} \in \widehat{B}_{k+l+4}$ such that the sequence of the left hand side is consistent,

$$
a_{k+1, l+1}^{\epsilon_{3}} \cdot\left(\sigma_{k+3}^{\epsilon_{2}^{\prime}}\right)^{-c} \cdot c_{k+2, l}^{\epsilon_{2}} \cdot\left(\sigma_{k+1}^{\epsilon_{1}^{\prime}}\right)^{c} \cdot c_{k, l}^{\epsilon_{1}}=c_{k, l}^{\epsilon_{3}^{\prime}} .
$$

Here $\epsilon_{3}^{\prime}$ is chosen to be $t\left(a_{k+1, l+1}^{\epsilon_{3}}\right)=t\left(c_{k, l}^{\epsilon_{3}^{\prime}}\right)$.

For $c \in \widehat{\mathbb{Z}}, c_{k+1, l+1}^{\epsilon_{1}} \in C, a_{k, l}^{\epsilon_{2}}, a_{k+2, l}^{\epsilon_{3}} \in A$ and $\sigma_{k+1}^{\epsilon_{2}^{\prime}}, \sigma_{k+3}^{\epsilon_{3}^{\prime}} \in \widehat{B}$ representing $\sigma_{k+1} \in \widehat{B}_{k+l+2}$ and $\sigma_{k+3} \in \widehat{B}_{k+l+4}$ such that the sequence of the left hand side is consistent,

$$
a_{k, l}^{\epsilon_{2}} \cdot\left(\sigma_{k+1}^{\epsilon_{2}^{\prime}}\right)^{-c} \cdot a_{k+2, l}^{\epsilon_{3}} \cdot\left(\sigma_{k+3}^{\epsilon_{3}^{\prime}}\right)^{c} \cdot c_{k+1, l+1}^{\epsilon_{1}}=a_{k, l}^{\epsilon_{1}^{\prime}}
$$

Here again $\epsilon_{1}^{\prime}$ is chosen to be $s\left(c_{k+1, l+1}^{\epsilon_{3}}\right)=s\left(a_{k, l}^{\epsilon_{1}^{\prime}}\right)$.

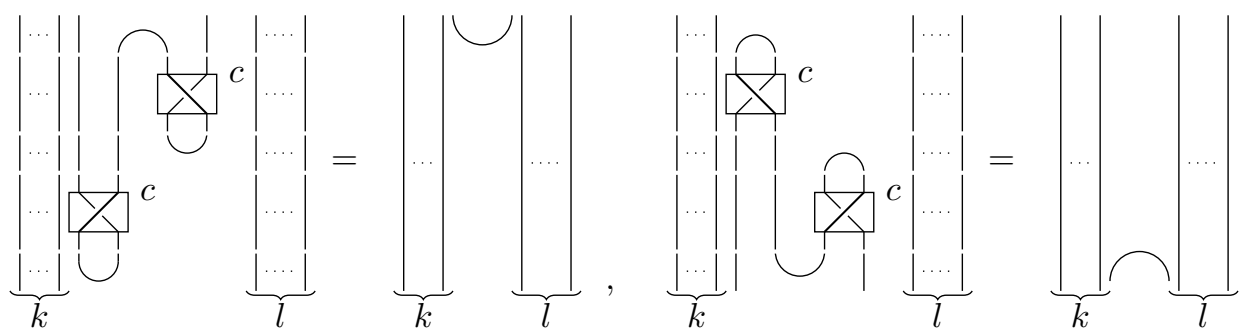

Figure B.1. (FT6): First framed Reidemeister move

We note that, in the first (resp. second) equation, $c_{k, l}^{\epsilon_{1}}=c_{k, l}^{\epsilon_{3}^{\prime}}$ (resp. $a_{k, l}^{\epsilon_{2}}=a_{k, l}^{\epsilon_{1}^{\prime}}$ ) if and only if $c \equiv 0(\bmod 2)$. It is easy to see that our previous (T6) implies (FT6).

Remark B.2. There is a natural projection $\widehat{\mathcal{F T}} \rightarrow \widehat{\mathcal{T}}$ and hence

$$
\widehat{\mathcal{F K}} \rightarrow \widehat{\mathcal{K}} \text {. }
$$

It is clear by definition.

Definition B.3. We introduce special tangles, twists, $\varphi^{\uparrow}$ and $\varphi^{\downarrow}$ by

$$
\varphi^{\uparrow}:=a_{1,0}^{\uparrow \curvearrowright} \cdot \sigma_{1}^{\uparrow \uparrow \downarrow} \cdot c_{1,0}^{\uparrow}, \quad \varphi^{\downarrow}:=a_{1,0}^{\downarrow \curvearrowleft} \cdot \sigma_{1}^{\downarrow \downarrow \uparrow} \cdot c_{1,0}^{\downarrow \smile} .
$$

The picture of $\varphi^{\uparrow}$ is depicted in the first term of Figure B.2.

It is an easy exercise to derive the lemma below from (FT1)-(FT6).

Lemma B.4. (1). The following equalities hold (cf. Figure B.2):

$$
\varphi^{\uparrow}=a_{0,1}^{\curvearrowleft \uparrow} \cdot \sigma_{2}^{\downarrow \uparrow \uparrow} \cdot c_{0,1}^{\smile \uparrow}=a_{0,1}^{\curvearrowright \uparrow} \cdot\left(\sigma_{2}^{-1}\right)^{\uparrow \uparrow \downarrow} \cdot c_{1,0}^{\uparrow \smile}=a_{0,1}^{\curvearrowleft \uparrow} \cdot\left(\sigma_{1}^{-1}\right)^{\uparrow \downarrow \uparrow} \cdot c_{1,0}^{\uparrow \smile} .
$$

(2). We have the following equalities (cf. Figure B.3)

$$
\left(\varphi^{\downarrow} \otimes \uparrow\right) \cdot \backsim=\left(\downarrow \otimes \varphi^{\uparrow}\right) \cdot \backsim, \quad \curvearrowright \cdot\left(\varphi^{\uparrow} \otimes \downarrow\right)=\curvearrowright \cdot\left(\uparrow \otimes \varphi^{\downarrow}\right) .
$$

We also have the same equalities obtained by reversing all arrows. 


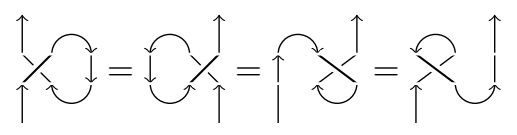

Figure B.2. twist $\varphi^{\uparrow}$

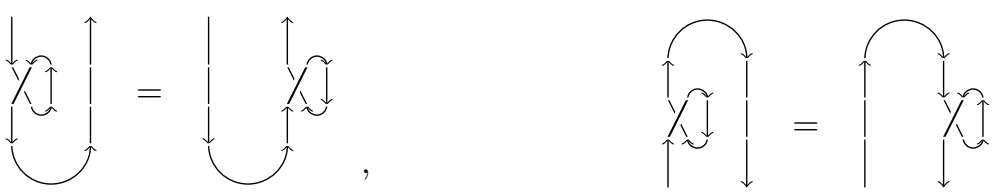

Figure B.3. Lemma B.4. (2).

The space $\widehat{\mathcal{F T}}$ (hence the subspace $\widehat{\mathcal{F K}}$ ) carries a structure of topological space by profinite topology on $\widehat{B}_{n}(n=1,2, \ldots)$ and the discrete topology on $A$ and on C.

We have a framed version of our Theorem $\mathrm{A}$ in $₫ 0$.

Theorem B.5. (1). The space $\widehat{\mathcal{F K}}$ carries a structure of a topological commutative monoid whose product structure

$$
\sharp^{\mathrm{fr}}: \widehat{\mathcal{F K}} \times \widehat{\mathcal{F K}} \rightarrow \widehat{\mathcal{F K}} .
$$

is given by the connected sum (2.1) for $K_{1}=\alpha_{m} \cdots \alpha_{1}$ and $K_{2}=\beta_{n} \cdots \beta_{1}$.

(2). Let $\mathcal{F} \mathcal{K}$ denote the set of framed isotopy classes of (topological) oriented framed knots 12 . Then there is a natural map

$$
h^{\mathrm{fr}}: \mathcal{F} \mathcal{K} \rightarrow \widehat{\mathcal{F K}} \text {. }
$$

The map is with dense image and is a monoid homomorphism with respect to the connected sum.

The map $h^{\text {fr }}$ should be conjectured to be injective by the same reason to Conjecture 2.12.

Proof. The proof can be done in an almost same way to that of Theorem 2.11 and 2.16. We outline it by strengthening a slight difference.

(1). Each framed isotopy class of profinite knot contains a profinite knot $K=$ $\gamma_{m} \cdots \gamma_{1}$ for some $m$ with $\left(\gamma_{m}, \gamma_{1}\right)=(\curvearrowleft, \cup)$ by $($ FT6) with $c \equiv 1(\bmod 2)$. Hence the connected sum could extend to $\widehat{\mathcal{F K}}$ once we have the well-definedness. To show the well-definedness, it is enough to show that $K_{1} \sharp K_{2}$ is framed isotopic to $K_{1}^{\prime} \sharp K_{2}$ when $K_{1}^{\prime}$ is obtained from $K_{1}$ by operating (FT3) or (FT6) on $\alpha_{1}$ by a similar reason to the proof of Theorem 2.16. Consider the latter case (FT6). It suffices to show that $K_{1} \sharp K_{2}$ is framed isotopic to $K_{3}=\alpha_{m} \cdots \alpha_{2} \cdot a_{1,1} \cdot \sigma_{3}^{-2 c} \cdot c_{2,0} \cdot \sigma_{1}^{2 c} \cdot \beta_{n-1} \cdots \beta_{1}$ for $c \in \widehat{\mathbb{Z}}$ (cf. Figure B.4). It can be shown in a similar way to Figure 2.16.

Next consider the former case (FT3). Since $K_{1}$ is a profinite knot, $m_{1}$ and $m_{2}$ are both 0. By the above argument in case (FT6), we may assume that both $T_{1}$ and $T_{2}$ in Figure 2.11 should start from $\mho$ (i.e. $\alpha_{1}=\beta_{1}=\mho$ ). Define $T$ as in Figure 2.17. A successive application of commutativity of profinite braids with $T$

\footnotetext{
12 Framed knot means a knot equipped with a framing, that is, a nonzero normal vector field on it.
} 


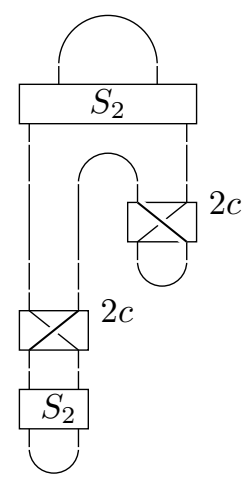

Figure B.4. $K_{3}$

shown in (FT4) and that of creations and annihilations with $T$ shown in Lemma B.6 lead the framed isotopy equivalence shown in Figure 2.18. Hence we get the the well-defined product $\sharp^{\mathrm{fr}}$.

The proofs of associativity, commutativity, continuity for $\sharp^{\mathrm{fr}}$ can be done in a quite same way to the proof of Theorem 2.16

(2). The result in Ba1 indicates that the set $\mathcal{F} \mathcal{T}$ of (framed) isotopy classes of framed tangles is described by the set of consistent finite sequences of fundamental tangles, elements of $A, B$ and $C$ modulo the (discrete) framed Turaev moves, which is equivalent to the moves replacing profinite tangles and braids by (discrete) tangles and braids in (FT1)-(FT6) and $c \in \widehat{\mathbb{Z}}$ by $c \in \mathbb{Z}$ in (FT6). Because we have a natural map $B_{n} \rightarrow \widehat{B}_{n}$ and the Turaev moves are special case of our 6 moves, we have a natural map $\mathcal{F} \mathcal{T} \rightarrow \widehat{\mathcal{F T}}$, which yields our map $h^{\text {fr }}$. It is easy to see that it is homomorphic and is with dense image.

A framed analogue of Lemma 2.17 and 2.18 hold.

Lemma B.6. Let $T$ be a profinite tangle $T$ with $s(T)=t(T)=\uparrow$.

(1). Its transpose $L$ (see. Lemma 2.17) is equal to $a_{1,0}^{\downarrow \curvearrowright} \cdot\left(e_{1}^{\downarrow} \otimes T \otimes e_{1}^{\downarrow}\right) \cdot c_{0,1}^{\cup \downarrow}$ (cf. Figure 2.20).

(2). The equalities in Figure 2.22 hold for $T$.

The same claim also holds for a profinite tangle $T$ with $s(T)=t(T)=\downarrow$ by reversing all arrows.

Proof. (1). A proof is depicted in Figure B.5. We use (FT6) in the first equality. The second and the fourth equalities follow from Lemma B.4.(1). The third equality is obtained by the 'commutativitiy' of twists with annihilation, creation and profinite braids assured by Lemma B.4.(2) and (FT4). The fifth equation follows from (FT2) and (FT4).

(2). It is a direct consequence of (1).

Definition B.7. The group of profinite framed knots Frac $\widehat{\mathcal{F K}}$ is defined to be the group of fraction of the monoid $\widehat{\mathcal{F K}}$ as in the same way to Definition 2.35

We also have a framed version of our Theorem B in 0.0 . 


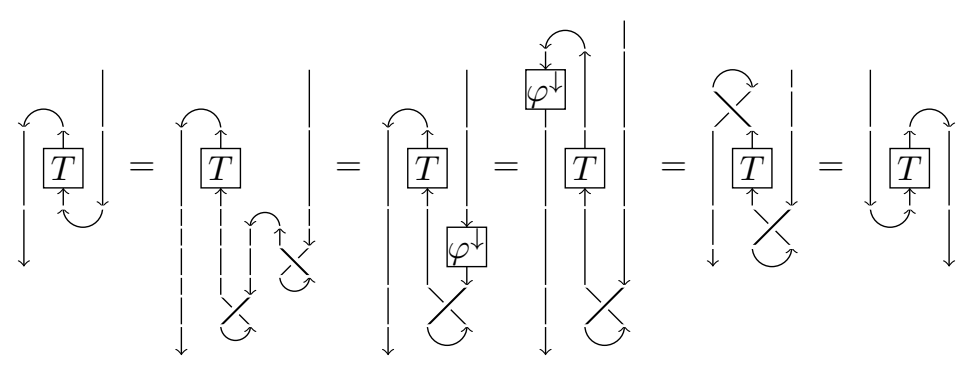

Figure B.5. Proof of Lemma B.6.(1).

Theorem B.8. Fix an embedding from the algebraic closure $\overline{\mathbb{Q}}$ of the rational number field $\mathbb{Q}$ into the complex number field $\mathbb{C}$. Then the group Frac $\widehat{\mathcal{F K}}$ of profinite knots admits a non-trivial topological $G_{\mathbb{Q}}$-module structure. Namely, there is a nontrivial continuous Galois representation on profinite knots

$$
\rho_{0}^{\text {fr }}: G_{\mathbb{Q}} \rightarrow \text { Aut Frac } \widehat{\mathcal{F K}} .
$$

Proof. This is achieved by establishing a consistent continuous action of the profinite Grothendieck-Teichmüller group $\widehat{G T}$ on Frac $\widehat{\mathcal{F K}}$ and using the inclusion from $G_{\mathbb{Q}}$ into $\widehat{G T}$.

We introduce this $\widehat{G T}$-action on Frac $\widehat{\mathcal{F K}}$ by using the action on each profinite fundamental tangle given in Definition 2.39, The proof of its well-definedness, i.e. to check that the action preserves (FT1)-(FT6), can be done in the same way to that of Theorem 2.41 but for (FT6), which is proved below: Let $\sigma=(\lambda, f) \in \widehat{G T}$. Let $r_{1}$ and $r_{2}$ be two profinite knots. Assume that $r_{1}$ is obtained from $r_{2}$ by a single operation of the move (FT6). Then by Proposition 1.16 and Proposition 2.42, we have

$$
\begin{aligned}
& \sigma\left(a_{k, l}^{\epsilon_{2}}\right) \cdot \sigma\left(\left(\sigma_{k+1}^{\epsilon_{2}^{\prime}}\right)^{-c}\right) \cdot \sigma\left(a_{k+2, l}^{\epsilon_{3}}\right) \cdot \sigma\left(\left(\sigma_{k+3}^{\epsilon_{3}^{\prime}}\right)^{c}\right) \cdot \sigma\left(c_{k+1, l+1}^{\epsilon_{1}}\right) \\
& =a_{k, l}^{\epsilon_{2}} \cdot f_{1 \cdots k, k+1, k+2}^{s\left(a_{k, k}^{\epsilon_{2}}\right)} \cdot f_{1 \cdots k, k+1, k+2}^{-1, s\left(a_{k, l}^{\epsilon_{2}}\right)} \cdot\left(\sigma_{k+1}^{\epsilon_{2}^{\prime}}\right)^{-c \lambda} \cdot f_{1 \cdots k, k+1, k+2}^{t\left(a_{k+2, l}^{\epsilon_{3}}\right)} \\
& \cdot a_{k+2, l}^{\epsilon_{3}} \cdot f_{1 \cdots k+2, k+3, k+4}^{s\left(a_{k+2, l}^{\epsilon_{3}}\right)} \cdot f_{1 \cdots k+2, k+3, k+4}^{-1, s\left(a_{k+2, l}^{\epsilon_{3}}\right)} \cdot\left(\sigma_{k+3}^{\epsilon_{3}^{\prime}}\right)^{c \lambda} \cdot f_{1 \ldots k+2, k+3, k+4}^{t\left(c_{k+1, l+1}^{\epsilon_{1}}\right)} \\
& \cdot f_{1 \cdots k+1, k+2, k+3}^{-1, t\left(c_{k+1, l+1}^{\epsilon_{1}}\right)} \cdot c_{k+1, l+1}^{\epsilon_{1}} \\
& =a_{k, l}^{\epsilon_{2}} \cdot\left(\sigma_{k+1}^{\epsilon_{2}^{\prime}}\right)^{-c \lambda} \cdot f_{1 \cdots k, k+1, k+2}^{t\left(a_{k+2, l}^{\epsilon_{3}}\right)} \cdot a_{k+2, l}^{\epsilon_{3}} \cdot\left(\sigma_{k+3}^{\epsilon_{3}^{\prime}}\right)^{c \lambda} \cdot f_{1 \cdots k+2, k+3, k+4}^{t\left(c_{k+1, l+1}^{\epsilon_{1}}\right)} \\
& \cdot f_{1 \cdots k+1, k+2, k+3}^{-1, t\left(c_{k+1, l+1}^{\epsilon_{1}}\right)} \cdot c_{k+1, l+1}^{\epsilon_{1}} \\
& =a_{k, l}^{\epsilon_{2}} \cdot\left(\sigma_{k+1}^{\epsilon_{2}^{\prime}}\right)^{-c \lambda} \cdot a_{k+2, l}^{\epsilon_{3}} \cdot\left(\sigma_{k+3}^{\epsilon_{3}^{\prime}}\right)^{c \lambda} \\
& \cdot f_{1 \cdots k+2, k+3, k+4}^{t\left(c_{k+1, l+1}^{\epsilon_{1}}\right)} \cdot f_{1 \cdots k, k+1, k+2}^{t\left(c_{k+1, l+1}^{\epsilon_{1}}\right)} \cdot f_{1 \cdots k+1, k+2, k+3}^{-1, t\left(c_{k+1, l+1}^{\epsilon_{1}}\right)} \cdot c_{k+1, l+1}^{\epsilon_{1}} \cdot
\end{aligned}
$$

By a successive application of (1.4),

$$
\begin{aligned}
=a_{k, l}^{\epsilon_{2}} & \cdot\left(\sigma_{k+1}^{\epsilon_{2}^{\prime}}\right)^{-c \lambda} \cdot a_{k+2, l}^{\epsilon_{3}} \cdot\left(\sigma_{k+3}^{\epsilon_{3}^{\prime}}\right)^{c \lambda} \\
& \cdot f_{1 \ldots k, k+1, k+2}^{t\left(c_{k+1, l+1}^{\epsilon_{1}}\right)} \cdot f_{1 \cdots k+1, k+2, k+3}^{-1, t\left(c_{k+1, l+1}^{\epsilon_{1}}\right)} \cdot f_{k+2, k+3, k+4}^{t\left(c_{k+1, l+1}^{\epsilon_{1}}\right)} \cdot c_{k+1, l+1}^{\epsilon_{1}} .
\end{aligned}
$$


By (FT4) and Lemma 1.8

$$
=a_{k, l}^{\epsilon_{2}} \cdot\left(\sigma_{k+1}^{\epsilon_{2}^{\prime}}\right)^{-c \lambda} \cdot f_{1 \ldots k, k+1, k+2}^{t\left(a_{k+2, l}^{\epsilon_{3}}\right)} \cdot a_{k+2, l}^{\epsilon_{3}} \cdot\left(\sigma_{k+3}^{\epsilon_{3}^{\prime}}\right)^{c \lambda} \cdot f_{k+2, k+3, k+4}^{t\left(c_{k+1, l+1}^{\epsilon_{1}}\right)} \cdot c_{k+1, l+1}^{\epsilon_{1}} .
$$

By Lemma B.10 twists $\left(\varphi^{\uparrow}\right)^{c}$ and $\left(\varphi^{\downarrow}\right)^{c}(c \in \widehat{\mathbb{Z}})$ 'commute' with profinite tangles. Thus

$$
\begin{aligned}
=a_{k, l}^{\epsilon_{2}} & \cdot\left(\sigma_{k+1}^{\epsilon_{2}^{\prime}}\right)^{-c \lambda} \cdot a_{k+2, l}^{\epsilon_{3}} \cdot\left(\sigma_{k+3}^{\epsilon_{3}^{\prime}}\right)^{c \lambda} \cdot c_{k+1, l+1}^{\epsilon_{1}} \cdot f_{1 \ldots k, k+1, k+2}^{s\left(\epsilon_{k, l}^{\epsilon_{1}^{\prime}}\right)} \\
& \cdot a_{k+2, l}^{\epsilon_{0}} \cdot f_{k+2, k+3, k+4}^{s\left(a_{k+2, l}^{\epsilon_{0}}\right)} \cdot c_{k+1, l+1}^{\epsilon_{0}^{\prime}}
\end{aligned}
$$

with appropriate orientations $\epsilon_{0}$ and $\epsilon_{0}^{\prime}$. Hence

$$
\begin{aligned}
& =a_{k, l}^{\epsilon_{1}^{\prime}} \cdot f_{1 \cdots k, k+1, k+2}^{s\left(a_{k, l}^{\epsilon_{1}^{\prime}}\right)} \cdot a_{k+2, l}^{\epsilon_{0}} \cdot f_{k+2, k+3, k+4}^{s\left(a_{k+2, l}^{\epsilon_{0}}\right)} \cdot c_{k+1, l+1}^{\epsilon_{0}^{\prime}} \\
& =\sigma\left(a_{k, l}^{\epsilon_{1}^{\prime}}\right) \cdot a_{k+2, l}^{\epsilon_{0}} \cdot f_{k+2, k+3, k+4}^{s\left(a_{k+2, l}^{\epsilon_{0}}\right)} \cdot c_{k+1, l+1}^{\epsilon_{0}^{\prime}} .
\end{aligned}
$$

Therefore we have $\sigma\left(r_{1}\right)=\sigma\left(r_{2}\right) \sharp \Lambda_{f}$. Because $\alpha\left(r_{1}\right)=\alpha\left(r_{2}\right)+1$, we may say that (B.2) is preserved by the $\widehat{G T}$-action. The proof for (B.1) can be done in the same way. Thus the first framed Reidemeister move (FT6) is preserved by the $\widehat{G T}$-action.

The rest part of our claim; continuity, non-triviality, etc, was shown in the proof of Theorem 2.41,(2)-(5).

Lemma B.9. The map sending $c \in \mathbb{N}$ to the $c$-th power $\left(\varphi^{\uparrow}\right)^{c} \in \widehat{\mathcal{F T}}$ of $\varphi^{\uparrow}$ extends additively and continuously into $c \in \widehat{\mathbb{Z}}$ and it is given by

$$
\left(\varphi^{\uparrow}\right)^{c}=a_{0,1} \cdot\left(\sigma_{1}^{-c}\right)^{\uparrow \downarrow \uparrow} \cdot c_{1,0}^{\uparrow} .
$$

with $a_{0,1}=a_{0,1}^{\curvearrowleft \uparrow}$ or $a_{0,1}^{\curvearrowright \uparrow}$ according to $c \equiv 1$ or $0(\bmod 2)$ (cf. Figure B.6).

The same statement also holds for $\varphi^{\downarrow}$.

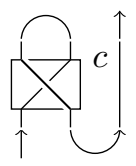

FiguRe B.6. $\left(\varphi^{\uparrow}\right)^{c}$

Proof. The equation (B.4) for $c \in \mathbb{N}$ can be shown by induction: Assume the validity for $c$ with $c \equiv 1(\bmod 2)$. Then

$$
\left(\varphi^{\uparrow}\right)^{c+1}=\left(\varphi^{\uparrow}\right)^{c} \cdot \varphi^{\uparrow}=a_{0,1}^{\curvearrowleft \uparrow} \cdot\left(\sigma_{1}^{-c}\right)^{\uparrow \downarrow \uparrow} \cdot c_{1,0}^{\uparrow} \cdot \varphi^{\uparrow},
$$

by Lemma B.4. (2)

$$
=a_{0,1}^{\curvearrowleft \uparrow} \cdot\left(e_{\downarrow} \otimes \varphi^{\uparrow} \otimes e_{\uparrow}\right) \cdot\left(\sigma_{1}^{-c}\right)^{\uparrow \downarrow \uparrow} \cdot c_{1,0}^{\uparrow},
$$

by Lemma B.4.(1)

$$
=a_{0,1}^{\curvearrowright \uparrow} \cdot\left(\sigma_{1}^{-c-1}\right)^{\uparrow \downarrow \uparrow} \cdot c_{1,0}^{\uparrow} .
$$


The case for $c$ with $c \equiv 0(\bmod 2)$ can be done in the same way. By the expression of (B.4), it is immediate to see that $\left(\varphi^{\uparrow}\right)^{c}$ consistently extends to the case for $c \in \widehat{\mathbb{Z}}$.

As an analogue of Lemma B.4 (2), we have

Lemma B.10. We have the following equalities

$$
\left(\left(\varphi^{\downarrow}\right)^{c} \otimes \uparrow\right) \cdot \backsim=\left(\downarrow \otimes\left(\varphi^{\uparrow}\right)^{c}\right) \cdot \cup, \quad \curvearrowright \cdot\left(\left(\varphi^{\uparrow}\right)^{c} \otimes \downarrow\right)=\curvearrowright \cdot\left(\uparrow \otimes\left(\varphi^{\downarrow}\right)^{c}\right)
$$

for $c \in \widehat{\mathbb{Z}}$. We also have the same equalities obtained by reversing all arrows.

Proof. A proof is depicted in Figure B.7. We note that we use Lemma B.4.(1) and (FT6) to deduce the second and the third equality respectively.

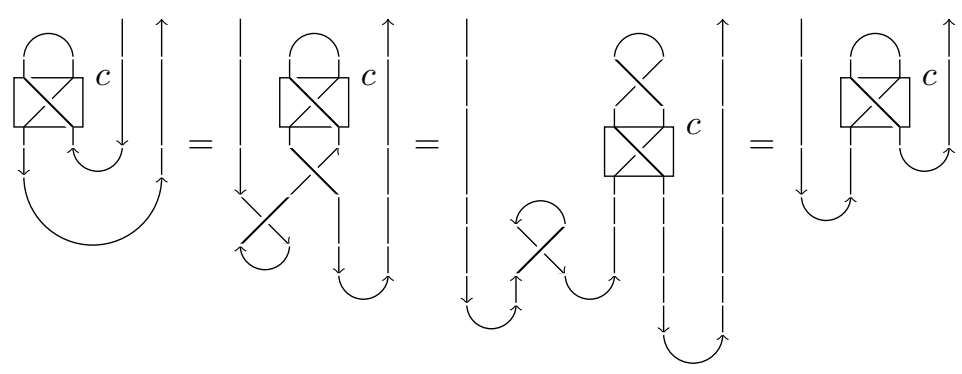

Figure B.7. Proof of Lemma B.10

We note that the $G_{\mathbb{Q}}$-module structure on the group Frac $\widehat{\mathcal{K}}$ of profinite knots given in Theorem $\mathrm{B}$ in $\oint 0$ is induced from that on the group Frac $\widehat{\mathcal{F K}}$ of profinite framed knots by (B.3).

Acknowledgements. The author would like to express his profound gratitude to Masanori Morishita who persistently encouraged this research. His thanks are also directed to Hiroshi Fujiwara who patiently helped him to draw many graphics in this paper, Hitoshi Murakami, Toshitake Kohno and Tetsuya Ito who generously explained him lots of valuable issues in low dimensional topology related to this paper. Part of the paper was written at Max Planck Institute for Mathematics. He also thanks the institute for its hospitality. This work was supported by Grantin-Aid for Young Scientists (A) 24684001. The referees efforts to make this paper better is gratefully acknowledged.

\section{REFERENCES}

[Ba1] Bar-Natan, D., Non-associative tangles, Geometric topology (Athens, GA, 1993), 139-183, AMS/IP Stud. Adv. Math., 2.1, Amer. Math. Soc., Providence, RI, 1997.

[Ba2] - Vassiliev homotopy string link invariants, J. Knot Theory Ramifications 4 (1995), no. $1,1332$.

[Be] Belyı̆, G. V., Galois extensions of a maximal cyclotomic field, (Russian) Izv. Akad. Nauk SSSR Ser. Mat. 43 (1979), no. 2, 267-276, 479.

[Bi] Birman, J. S., Braids, links, and mapping class groups, Annals of Mathematics Studies, No. 82. Princeton University Press, Princeton, N.J.; University of Tokyo Press, Tokyo, 1974.

[CDM] Chmutov, S., Duzhin, S. and Mostovoy, J., Introduction to Vassiliev Knot Invariants, Cambridge University Press, 2012. 
[De] Deligne, P., Le groupe fondamental de la droite projective moins trois points, Galois groups over $\mathbb{Q}$ (Berkeley, CA, 1987), 79-297, Math. S. Res. Inst. Publ., 16, Springer, New YorkBerlin, 1989.

[Dr1] Drinfel'd, V. G., Quasi-Hopf algebras, Leningrad Math. J. 1 (1990), no. 6, 1419-1457.

[Dr2] On quasitriangular quasi-Hopf algebras and a group closely connected with $\operatorname{Gal}(\overline{\mathbb{Q}} / \mathbb{Q})$, Leningrad Math. J. 2 (1991), no. 4, 829-860.

[F1] Furusho, H., Pentagon and hexagon equations, Annals of Mathematics, Vol. 171 (2010), No. 1, 545-556.

[F2] , Galois action on knots II: Proalgebraic string links and knots, preprint, arXiv: 1405.4575

[GW] Gannon, T. and Walton, M. A., Galois relations on knot invariants, Lett. Math. Phys. 38 (1996), no. 2, 185-194.

[G1] Grothendieck, A., Revêtement Étales et Groupe Fondamental (SGA 1), Lecture Notes in Math. 224, Springer-Verlag 1971.

[G2] Esquisse d'un programme, 1983, available on pp. 5-48. London Math. Soc. LNS 242, Geometric Galois actions, 1, Cambridge Univ.

[HL] Habegger, N. and Lin, X.-S., The classification of links up to link-homotopy, J. Amer. Math. Soc. 3 (1990), no. 2, 389419.

[H] Hain, R., The Hodge de Rham theory of relative Malcev completion, Ann. Sci. École Norm. Sup. (4) 31, no. 1, 4792, 1998.

[I1] Ihara, Y., Braids, Galois groups, and some arithmetic functions, Proceedings of the International Congress of Mathematicians, Vol. I, II (Kyoto, 1990), 99-120, Math. Soc. Japan, Tokyo, 1991.

[I2] - On the embedding of $\operatorname{Gal}(\overline{\mathbb{Q}} / \mathbb{Q})$ into $\widehat{G T}$, with an appendix: the action of the absolute Galois group on the moduli space of spheres with four marked points by Emsalem, M. and Lochak, P. London Math. Soc. Lecture Note Ser., 200, The Grothendieck theory of dessins d'enfants (Luminy, 1993), 289-321, Cambridge Univ. Press, Cambridge, 1994.

[IM] - and Matsumoto, M., On Galois actions on profinite completions of braid groups, Recent developments in the inverse Galois problem (Seattle, WA, 1993), 173-200, Contemp. Math., 186, Amer. Math. Soc., Providence, RI, 1995.

[JS] Joyal, A. and Street, R., Braided tensor categories, Adv. Math.102 (1993), no. 1, 20-78.

[Kap] Kapranov, M. M., Analogies between the Langlands correspondence and topological quantum field theory, Functional analysis on the eve of the 21st century, Vol. 1 (New Brunswick, NJ, 1993), 119-151, Progr. Math., 131, Birkhäuser Boston, Boston, MA, 1995.

[K] Kassel, C., Quantum groups, Graduate Texts in Mathematics, 155, Springer-Verlag, New York, 1995.

[KT] and Turaev, V., Chord diagram invariants of tangles and graphs, Duke Math. J. 92 (1998), no. 3, 497-552.

[Ko] Kontsevich, M., Vassiliev's knot invariants, I. M. Gelfand Seminar, 137-150, Adv. Soviet Math., 16, Part 2, Amer. Math. Soc., Providence, RI, 1993.

[LM] Le, T. T. Q. and Murakami, J., The universal Vassiliev-Kontsevich invariant for framed oriented links, Compositio Math.102 (1996), no. 1, 41-64.

[Lic] Lickorish, W. B. R., An introduction to knot theory, Graduate Texts in Mathematics, 175, Springer-Verlag, New York, 1997.

[Lin] Lin, X.-S., Power series expansions and invariants of links, Geometric topology (Athens, GA, 1993), 184202, AMS/IP Stud. Adv. Math., 2.1, Amer. Math. Soc., Providence, RI, 1997.

[LS] Lochak, P. and Schneps, L., The Grothendieck-Teichmüller group and automorphisms of braid groups, The Grothendieck theory of dessins d'enfants (Luminy, 1993), 323-358, London Math. Soc. Lecture Note Ser., 200, Cambridge Univ. Press, Cambridge, 1994.

[Ma] Mazur, B., Primes, knots and Po, lecture note in the conference in celebration of the 80th birthday of Valentin Poenaru, held in Autrans, France July 1-6, 2012.

[Mi1] Milnor, B., Link groups, Ann. of Math. 59 (1954), 177-195.

[Mi2] Isotopy of links, Algebraic Geometry and Topology, Princeton Univ. Press, Princeton, NJ, 1957.

[Mo] Morishita, M., Knots and primes, An introduction to arithmetic topology, Universitext. Springer, London, 2012. 
[O1] Ohtsuki, T., Quantum invariants. A study of knots, 3-manifolds, and their sets, Series on Knots and Everything, 29, World Scientific Publishing Co., Inc., River Edge, NJ, 2002.

[O2] , Problems on invariants of knots and 3-manifolds, Geom. Topol. Monogr., 4, Invariants of knots and 3-manifolds (Kyoto, 2001), i-iv, 377-572, Geom. Topol. Publ., Coventry, 2002.

[P] Piunikhin, S., Combinatorial expression for universal Vassiliev link invariant, Comm. Math. Phys. 168 (1995), no. 1, 1-22.

[R] Reznikov, A., Three-manifolds class field theory (homology of coverings for a nonvirtually b1-positive manifold), Selecta Math. (N.S.) 3 (1997), no. 3, 361-399.

[RZ] Ribes, L. and Zalesskii, P., Profinite groups, Second edition. Ergebnisse der Mathematik und ihrer Grenzgebiete. 3. Folge. A Series of Modern Surveys in Mathematics, 40, SpringerVerlag, Berlin, 2010.

[S] Schneps, L., The Grothendieck-Teichmuller group $\widehat{G T}$ : a survey, 183-203 in Geometric Galois Actions I, LMS Lecture Notes 242, Cambridge U. Press, 1997.

[Tr] Traczyk, P., A new proof of Markov's braid theorem, Knot theory (Warsaw, 1995), 409419, Banach Center Publ., 42, Polish Acad. Sci., Warsaw, 1998.

[Tu] Turaev, V. G., Operator invariants of tangles, and R-matrices, Izv. Akad. Nauk SSSR Ser. Mat. 53 (1989), no. 5, 1073-1107, 1135; translation in Math. USSR-Izv. 35 (1990), no. 2, 411-444

[V] Vogel, P., Representation of links by braids: a new algorithm, Comment. Math. Helv. 65 (1990), no. 1, 104-113.

[Y] Yamada, S., The minimal number of Seifert circles equals the braid index of a link, Invent. Math. 89 (1987), no. 2, 347-356.

Graduate School of Mathematics, Nagoya University, Furo-cho, Chikusa-ku, Nagoya, 464-8602, JAPAN

E-mail address: furusho@math.nagoya-u.ac.jp 